\title{
FLOER MINI-MAX THEORY, THE CERF DIAGRAM, AND THE SPECTRAL INVARIANTS
}

\author{
YONG-GEUN OH
}

\begin{abstract}
The author previously defined the spectral invariants, denoted by $\rho(H ; a)$, of a Hamiltonian function $H$ as the mini-max value of the action functional $\mathcal{A}_{H}$ over the Novikov Floer cycles in the Floer homology class dual to the quantum cohomology class $a$. The spectrality axiom of the invariant $\rho(H ; a)$ states that the mini-max value is a critical value of the action functional $\mathcal{A}_{H}$. The main purpose of the present paper is to prove this axiom for nondegenerate Hamiltonian functions in irrational symplectic manifolds $(M, \omega)$. We also prove that the spectral invariant function $\rho_{a}: H \mapsto \rho(H ; a)$ can be pushed down to a continuous function defined on the universal (étale) covering space $\widetilde{\operatorname{Ham}}(M, \omega)$ of the group $\operatorname{Ham}(M, \omega)$ of Hamiltonian diffeomorphisms on general $(M, \omega)$. For a certain generic homotopy, which we call a Cerf homotopy $\mathcal{H}=\left\{H^{s}\right\}_{0 \leq s \leq 1}$ of Hamiltonians, the function $\rho_{a} \circ \mathcal{H}: s \mapsto \rho\left(H^{s} ; a\right)$ is piecewise smooth away from a countable subset of $[0,1]$ for each non-zero quantum cohomology class $a$.

The proof of this nondegenerate spectrality relies on several new ingredients in the chain level Floer theory, which have their own independent interest: a structure theorem on the Cerf bifurcation diagram of the critical values of the action functionals associated to a generic one-parameter family of Hamiltonian functions, a general structure theorem and the handle sliding lemma of Novikov Floer cycles over such a family and a family version of new transversality statements involving the Floer chain map, and many others. We call this chain level Floer theory as a whole the Floer mini-max theory.
\end{abstract}

\section{Contents}

$\S 1$. Introduction and the main results

$\S 2$. Preliminary and review of the rational case

2.1. Novikov Floer cycles and spectral invariants

2.2. Review of the rational case

Received September 6, 2007.

2000 Mathematics Subject Classification. 53D35, 53D40.

Key words and phrases. irrational symplectic manifolds, Hamiltonian functions, action functional, Cerf bifurcation diagram, sub-homotopies, tight Floer cycles, handle sliding lemma, spectral invariants, spectrality axiom.

Partially supported by the US NSF grant \#DMS 0203593 \& 0503954. 
$\S 3$. The Cerf bifurcation diagram

3.1. Generic bifurcations of the critical set of the action functional

3.2. The Cerf bifurcation diagram

$\S 4$. Sub-homotopies and transversality

4.1 Definition of the Floer boundary map, re-visited

4.2. Definition of the Floer chain map, re-visited

4.3. Transversality of sub-homotopies

$\S 5$. Composition law of Floer's chain maps, re-visited

$\S 6$. Structure of Novikov Floer cycles in a Cerf family

$\S 7$. Handle sliding lemma and sub-homotopies

$\S 8$. Parametric stability of tightness of Novikov Floer cycles

$\S 9$. Proof of the nondegenerate spectrality

$\S 10$. Spectral invariants of Hamiltonian diffeomorphisms

$\S 11$. Applications to Hofer's geometry

Appendix

\section{$\S 1$. Introduction and the main results}

Let $(M, \omega)$ be a closed symplectic manifold and $\operatorname{Ham}(M, \omega)$ be the group of smooth Hamiltonian diffeomorphisms as a subgroup of symplectic diffeomorphisms $\operatorname{Symp}(M, \omega)$ with the smooth topology on it. In a previous paper [18], to each one-periodic time-dependent normalized Hamiltonian function $H: S^{1} \times M \rightarrow \mathbb{R}$, we associated a family of symplectic invariants $\rho(H ; a)$ parameterized by the quantum cohomology classes $0 \neq a \in Q H^{*}(M)$, which we call the spectral invariants of $H$. These are the mini-max values of the action functional

$$
\begin{gathered}
\mathcal{A}_{H}: \widetilde{\Omega}_{0}(M) \rightarrow \mathbb{R} \\
\mathcal{A}_{H}([\gamma, w])=-\int w^{*} \omega-\int_{0}^{1} H(t, \gamma(t)) d t
\end{gathered}
$$

over the semi-infinite Floer cycles in the homology class 'dual' to the quantum cohomology class $a$. (See [18] for a precise meaning of the notion of the 'dual' used here.) Here $\Omega_{0}(M)$ is the set of contractible loops on $M$ and $\widetilde{\Omega}_{0}(M)$ is the standard $\Gamma$-covering space [10]: Two pairs $(\gamma, w)$ and $\left(\gamma, w^{\prime}\right)$ with $w, w^{\prime}$ : $D^{2} \rightarrow M$ satisfying $\partial w=\partial w^{\prime}=\gamma$ are equivalent if they satisfy

$$
\omega\left(w^{\prime} \# \bar{w}\right)=0 \quad \text { and } \quad c_{1}\left(w^{\prime} \# \bar{w}\right)=0 .
$$

We denote by $[\gamma, w]$ the equivalence class of $(\gamma, w)$ and by $\widetilde{\Omega}_{0}(M)$ the set of equivalence classes $[\gamma, w]$. We provide $\widetilde{\Omega}_{0}(M)$ with the quotient topology 
induced from the natural $C^{\infty}$ topology of the set of pairs $(z, w)$. We denote the (étale) covering group of $\pi: \widetilde{\Omega}_{0}(M) \rightarrow \Omega_{0}(M)$ by $\Gamma$, which is defined by

$$
\Gamma=\frac{\pi_{2}(M)}{\left.\left.\operatorname{ker} \omega\right|_{\pi_{2}(M)} \cap \operatorname{ker} c_{1}\right|_{\pi_{2}(M)}}
$$

and denote

$$
\operatorname{Spec}(H)=\left\{\mathcal{A}_{H}([z, w]) \mid \dot{z}=X_{H}(z)\right\}
$$

which is nothing but the set of critical values of the action functional $\mathcal{A}_{H}$.

The spectral invariants $\rho(H ; a)$ can be regarded as the invariants of the associated Hamiltonian paths

$$
\lambda=\phi_{H}: t \mapsto \phi_{H}^{t}
$$

We say that two Hamiltonians $H, H^{\prime} \mapsto \phi$ are equivalent if the corresponding Hamiltonian paths $\phi_{H}$ and $\phi_{H^{\prime}}$ are path homotopic in $\operatorname{Ham}(M, \omega)$. We denote by $[\phi, H]$ its path homotopy class, and by $\widetilde{\operatorname{Ham}}(M, \omega)$ the set of path homotopy classes. $\widetilde{\operatorname{Ham}}(M, \omega)$ is the universal covering space.

We assume that all Hamiltonian functions $H$ are normalized by the condition

$$
\int H_{t} d \mu=0 \text { for all } t \in[0,1]
$$

and denote by

$$
\mathcal{H}_{m}(M)=C_{m}^{\infty}\left(S^{1} \times M\right)
$$

the set of normalized one-periodic functions. Here ' $m$ ' stands for the term 'mean zero'. This set has one-one correspondence with the set

$$
\mathcal{P}(\operatorname{Ham}(M, \omega), i d)
$$

of Hamiltonian paths in $\operatorname{Ham}(M, \omega)$ based at the identity.

We recall that a symplectic manifold $(M, \omega)$ is called rational if its period group

$$
\Gamma_{\omega}:=\omega(\Gamma)=\left\{\omega(A) \mid A \in \pi_{2}(M)\right\}
$$

is discrete. In [18], for the rational symplectic manifold $(M, \omega)$, we have proved

$$
\rho(H ; a)=\rho\left(H^{\prime} ; a\right)
$$

when $H, H^{\prime} \mapsto \phi$ and $[\phi, H]=\left[\phi, H^{\prime}\right]$, irrespective of nondegeneracy of Hamiltonians. In particular, the function $\rho$ induces a well-defined function

$$
\rho: \widetilde{\operatorname{Ham}}(M, \omega) \times Q H^{*}(M) \rightarrow \mathbb{R}
$$

by setting $\rho(\widetilde{\phi} ; a):=\rho(H ; a)$. Our proof in [18] of (1.3) for the rational case relies on the following 5 facts:

(1) The set $\operatorname{Spec}(H) \subset \mathbb{R}$, which is the set of critical values of the action functional $\mathcal{A}_{H}$ is a set of measure zero (see [16, Lemma 2.2]). 
(2) For any two Hamiltonian functions $H, H^{\prime} \mapsto \phi$ such that $[\phi, H]=$ $\left[\phi, H^{\prime}\right]$, we have

$$
\operatorname{Spec}(H)=\operatorname{Spec}\left(H^{\prime}\right)
$$

as a subset of $\mathbb{R}$ provided $H, H^{\prime}$ satisfy the normalization condition (1.2) (see [16] for the proof).

(3) The function $H \mapsto \rho(H ; a)$ is continuous with respect to the smooth topology on $C_{m}^{\infty}\left(S^{1} \times M\right)$ (see [18] for its proof).

(4) (Spectrality Axiom) For any $H$ and $a$, we have

$$
\rho(H ; a) \in \operatorname{Spec}(H) .
$$

(5) The only continuous functions on a connected space (e.g., the interval $[0,1])$ to $\mathbb{R}$, whose values lie in a measure zero subset, are constant functions.

The author previously proved the facts (1)-(3) for the general cases in a series of papers [15, 17, 18], especially including the irrational cases. However we were able to prove the spectrality axiom (4) only for the rational case at the time of writing the paper [18]. In this paper, we prove this spectrality axiom for nondegenerate Hamiltonian functions in the general symplectic manifolds, especially for the irrational symplectic manifolds.

Theorem I (Nondegenerate spectrality). Let $(M, \omega)$ be an arbitrary closed symplectic manifold. For any one-periodic nondegenerate Hamiltonian function $H: S^{1} \times M \rightarrow \mathbb{R}, \rho(H ; a)$ is a critical value of $\mathcal{A}_{H}$, i.e.,

$$
\rho(H ; a) \in \operatorname{Spec}(H)
$$

for any given quantum cohomology class $0 \neq a \in Q H^{*}(M)$.

We cannot prove the spectrality axiom for general degenerate Hamiltonian functions. We suspect that this is indeed not the case when degeneracy of the Hamiltonian function is severe. It is an interesting problem to prove or to find a counter example of the spectrality axiom.

In fact, we prove the following stronger theorem for the nondegenerate case.

Theorem II (Homological essentialness). Suppose that $H$ is nondegenerate and $a$ be any non-zero quantum cohomology class. Then $\rho(H ; a)$ is a homologically essential critical value of $\mathcal{A}_{H}$, in that there is a (and so any) generic choice of $J$ such that there is a Novikov Floer cycle $\alpha$ of $(H, J)$ satisfying $[\alpha]=a^{b}$ and

$$
\rho(H ; a)=\lambda_{H}(\alpha)
$$

We refer to Section 2 for the definition of a Novikov Floer cycle $\alpha$ of the pair $(H, J)$ and its level $\lambda_{H}(\alpha)$.

Theorem I, which we call the nondegenerate spectrality axiom, is an essential ingredient to push down the function $\rho_{a}: H \mapsto \rho(H ; a)$ to a continuous function 
on the universal covering space $\widetilde{\operatorname{Ham}}(M, \omega)$. We refer to [16]-[19] for a more detailed study of spectral invariants and their applications.

Because we do not know the validity of spectral axiom for degenerate Hamiltonians, the scheme of the proof used to prove (1.3) for the rational $(M, \omega)$ cannot be applied to degenerate Hamiltonians. In this regard, the following result is still a non-trivial theorem to prove.

Theorem III (Homotopy invariance). For any pair $(H, K)$, not necessarily nondegenerate, satisfying $H \sim K$, we have

$$
\rho(H ; a)=\rho(K ; a) .
$$

To prove Theorem III for degenerate Hamiltonians, we will use Theorem I together with continuity property of the spectral invariant function

$$
H \mapsto \rho(H ; a)
$$

and some simple calculation of the Hamiltonian algebra in the spirit of the proof of [15, Lemma 5.1] or [18, Theorem 5.1]. This theorem immediately gives rise to the following theorem.

Theorem IV. Let $\widetilde{\operatorname{Ham}}(M, \omega)$ be the above universal (étale) covering space and equip it with the quotient topology induced from $\mathcal{P}(\operatorname{Ham}(M, \omega), i d)$. Then the function

$$
\rho_{a}: \widetilde{\operatorname{Ham}}(M, \omega) \rightarrow \mathbb{R}
$$

defined by $\rho_{a}(\widetilde{\phi}):=\rho(H ; a)$ for a $H$ satisfying $[H]=\widetilde{\phi}$ is a continuous function in the natural topology of $\widetilde{\operatorname{Ham}}(M, \omega)$.

In the proof [18] of the general spectrality axiom for the rational case, we have used the fact, in an essential way, that the period group $\omega(\Gamma)$ is discrete and so $\operatorname{Spec}(H)$ is a closed subset of $\mathbb{R}$. For the irrational case, the argument for the rational case cannot be applied because $\operatorname{Spec}(H) \subset \mathbb{R}$ is not a closed subset but a dense subset (of measure zero) of $\mathbb{R}$. In the classical mini-max theory (see [1] for example) where the global gradient flow of the functional exists, a proof of the convergence result of the mini-max sequence heavily relies on the Palais-Smale type condition and the deformation lemma. In our case the global flow does not exist and the set of critical values is dense and hence there is no way to deform the space itself. Therefore in the point of view of the critical point theory, the action functional $\mathcal{A}_{H}$ on an irrational manifold $(M, \omega)$ belongs to the highly pathological realm.

To overcome these difficulties and prove criticality of the mini-max value $\rho(H ; a)$, on an irrational symplectic manifold $(M, \omega)$, we will work with the relevant mini-max cycles instead of either trying to deform the whole space or trying to prove convergence of the mini-max sequence of individual critical points. For this purpose, in the nondegenerate case, we use some intricate arguments involving the Novikov Floer cycles and the Floer chain map in the 
context of the chain level Floer theory. The proof in turn relies on a structure theorem of the Cerf bifurcation diagram of the action functional, a careful re-examination of the whole construction of the basic operators in the Floer homology theory and the composition law of the Floer chain map, and a structure theorem of general Novikov Floer cycles in a generic one-parameter family of Hamiltonian functions. This latter structure theorem, Theorem 6.7, is closely related to the picture arising in the First Cancellation Theorem in the classical Morse theory (See [13] and Section 5 for more explanations).

As a byproduct of our proof, we also prove the following piecewise smoothness of the spectral invariants under a Cerf homotopy of Hamiltonians. (See Definition 3.8 for the definition of Cerf homotopy and Theorem 9.5 for a more precise description of non-differentiable points.)

Theorem V. Let $\mathcal{H}=\left\{H^{s}\right\}_{0 \leq s \leq 1}$ be a smooth Cerf homotopy of Hamiltonians. Then the function

$$
s \in[0,1] \mapsto \rho\left(H^{s} ; a\right)
$$

is continuous piecewise-smooth with a countable number of non-differentiable points.

One may hope to use some limiting argument to study the degenerate cases as in the rational case. (See Section 2 for the limiting arguments.) This attempt meets a serious difficulty in the irrational case. The difficulty in proving existence of such a critical point $[z, w]$ by a limiting argument, does not lie in the part of periodic orbit $z$ but lies in the part $w$, because the structure of the covering group $\Gamma$ or rather its image $\Gamma_{\omega} \subset \mathbb{R}$, the period group of $(M, \omega)$, of the evaluation homomorphism $[\omega]: \Gamma \rightarrow \mathbb{R}$ is much more complex for the irrational case than for the rational case.

Besides the proof of the main theorem, in the course of our proof of the spectrality axiom for an irrational $(M, \omega)$, we prove many basic results in the Floer theory itself which seem to touch the heart of the chain level theory in the way Smale's handle body theory does in the classical Morse theory [13]. We expect that these will bring further consequences to symplectic topology in the future.

It is instructive to compare the ways how we maneuver the Floer cycles in [15] and in the present paper. One of the important matters in our chain level theory is to transfer a Floer cycle of one Hamiltonian to that of the other Hamiltonian along a given homotopy $\mathcal{H}=\{H(\eta)\}_{0<\eta<1}$ of Hamiltonian functions in a way that we can control the amount of 'sliding of handles', i.e., so that we can control the levels of the cycles during the transfer. For this purpose, we used a piecewise linear homotopy in [15], which we call the adiabatic homotopy. On the other hand, in the present paper, we use sub-homotopies of $(\mathcal{H}, j)$. (See Definition 3.9 for the definition of sub-homotopy.) For a given homotopy

$$
(\mathcal{H}, j):[0,1] \rightarrow C^{\infty}\left([0,1], \mathcal{H}_{m}(M)\right) \times C^{\infty}\left([0,1], j_{\omega}\right)
$$


we call $\left(\mathcal{H}_{\eta \eta^{\prime}}, j_{\eta \eta^{\prime}}\right)$ a $s u b$-homotopy of $(\mathcal{H}, j)$ for $0 \leq \eta, \eta \leq 1$, where the latter is defined by the homotopy

$$
\mathcal{H}_{\eta \eta^{\prime}}: s \mapsto H\left((1-s) \eta+s \eta^{\prime}\right), \quad j_{\eta \eta^{\prime}}: s \mapsto j\left((1-s) \eta+s \eta^{\prime}\right)
$$

for $s \in[0,1]$. Although this difference is marginal in the rational case, it turns out to be an essential improvement for the irrational case. In fact, our usage of sub-homotopies is one essential ingredient that enables us to prove certain continuity property of the levels of the transferred Floer cycles along the given homotopy $\mathcal{H}$. (See Proposition 8.4 and Lemma 8.5.) Such a continuity property would have been impossible to prove if we had used the adiabatic homotopy as in [15] due to the fact that $\operatorname{Spec}(H)$ is a dense subset of $\mathbb{R}$ : Since $\operatorname{Spec}(H)$ is dense, however fine a partition of $[0,1]$ we would choose, the levels of cycles could slide down to a lower level in each step of transfer along the piecewiselinear homotopy. As a result, we would not have been able to control the amount of handle sliding in the end if we used the piecewise-linear homotopy which only approximates the given homotopy $\mathcal{H}$. In hindsight, this is a natural thing to do in the point of view of Hamiltonian fibrations, which we will elaborate further elsewhere. However since we use sub-homotopies of a fixed homotopy of the pair $(\mathcal{H}, j)$, we have much less freedom to achieve transversality than the general case unlike when we use the adiabatic homotopies in [15] and so need to prove the transversality required to study such sub-homotopies. This leads us to a novel transversality statement (Theorem 4.6) which has not appeared in the previous literature and which is one of the essential ingredients in our proof.

A brief description of contents of the paper is in order. After a review of the proof for the rational case from [18] in Section 2, the rest of the paper deals with general symplectic manifolds, which are not necessarily rational. In Section 3, we prove a structure theorem of the Cerf bifurcation diagram of the action functionals for a generic one-parameter family of Hamiltonians connecting two nondegenerate Hamiltonians $H_{1}$ and $H_{2}$. In Section 4 , we study the transversality issue of sub-homotopies of the given homotopy or a one-parameter family $(\mathcal{H}, j)$ consisting of Hamiltonians and almost complex structures, and prove the main theorem, Theorem 4.6, in that regard. In Section 5, we carefully describe the gluing construction needed for the proof of the composition law of the Floer's chain maps in a way that will be used in our proof. In Section 6, we prove a structure theorem of Novikov Floer cycles over a one-parameter family of Hamiltonian functions. In Section 7, we recall the handle sliding lemma from [15] and provide a version thereof in terms of the sub-homotopy, instead of the adiabatic homotopy used in [15]. In Section 8 , we prove a general parametric stability theorem of the tightness of Novikov Floer cycles under a Cerf homotopy. As a by-product of the this proof, we derive Theorem $\mathrm{V}$ too. After all these preparations, we carry out the proof of Theorem II (and hence of Theorem I as a corollary) for the nondegenerate case in Section 9. In Section 10, we explain how we push down the spectral 
invariants to $\widetilde{\operatorname{Ham}}(M, \omega)$ as a continuous function in the natural topology on it, and in Section 11 we provide two immediate applications to Hofer's geometry of the Hamiltonian diffeomorphism group.

To make the main stream of arguments transparent without bogging down with technicalities involved with the transversality problem, as in [18, 19], we will assume that $(M, \omega)$ is strongly semi-positive, i.e., satisfies the condition that there is no spherical homology class $A$ satisfying

$$
\omega(A)>0 \text { and } 2-n \leq c_{1}(A)<0 .
$$

We will remove this assumption imposed in $[18,19]$ and in the current paper all at once in a sequel to these papers. The spectrality axiom was first announced in the author's preprint "Mini-max theory, spectral invariants and geometry of the Hamiltonian diffeomorphism group" (ArXiv:math.SG/0206092) in general. Since then, three papers, $[18,19]$ and the present paper, have grown out of the paper. Except the spectrality axiom for degenerate Hamiltonians in irrational symplectic manifolds and the treatment of the case that is not strongly semipositive, all the results in this preprint are now proved in the three papers with complete details and some corrections.

Finally we would like to point out that the spectrality axiom, or (1.3), is a crucial ingredient in Entov's work [4] in his applications of spectral invariants to the study of the quasimorphisms and the commutator length of Hamiltonian diffeomorphisms. A proof of the spectrality axiom for the nondegenerate case is outlined in [4, Section 3]. (See Part 4 of the page 76 of [4].) We emphasize that the standard argument used in the outline cannot be applied to the irrational symplectic manifolds because the set of critical values of the action functional is dense and the argument of 'pushing down the cycles under the flow' cannot easily go through in the irrational case, as we had mentioned before.

Recently Usher [22] gave an algebraic proof of the main theorem, Theorem II in a general abstract context of Floer homology.

We thank the Korea Institute for Advanced Study for providing the financial support and excellent research environment during the writing of the present paper. We also thank M. Usher for pointing out an incorrect statement in Theorem 3.7 (3) in the previous version of this paper and sending his preprint [22] : This results in our modification of the proof of Theorem 8.3 and that of Step 2 and 3 in Section 9 from the previous proofs thereof. Our usage of Proposition 8.8 is partly influenced by Usher's paper [22], where a similar statement is an important ingredient in his algebraic proof of Theorem II. We also thank him for many helpful comments during our preparation of this version of the paper.

\section{Notations}

(1) $\mathcal{J}_{\omega}=$ the set of $\omega$-compatible almost complex structures.

(2) $J=\left\{J_{t}\right\}_{0 \leq t<1}$ is a smooth one-periodic family with $J_{0}=J_{1}$, and $j_{\omega}$ is the set of such $J$ 's. We just denote $j_{\omega}=C^{\infty}\left(S^{1}, \mathcal{J}_{\omega}\right)$. 
(3) $\mathcal{P}\left(j_{\omega}\right)=C^{\infty}\left([0,1], j_{\omega}\right)$. We denote by $j$ a general element of $\mathcal{P}\left(j_{\omega}\right)$.

(4) $H: S^{1} \times M \rightarrow \mathbb{R}$ is a one-periodic family of functions that satisfy the normalization condition $\int_{M} H_{t} d \mu=0$, where $d \mu$ is the Liouville measure. We denote by $\mathcal{H}_{m}(M)=C_{m}^{\infty}\left(S^{1} \times M\right)$ the set of such $H$ 's.

(5) $\mathcal{H}=\{H(\eta)\}_{0 \leq \eta \leq 1}$ is a one-parameter family of $H$ 's mentioned in (4). We denote the set of such $\mathcal{H}^{\prime}$ 's by $\mathcal{P}\left(\mathcal{H}_{m}(M)\right)=C^{\infty}\left([0,1], \mathcal{H}_{m}(M)\right)$.

(6) $\Gamma_{\omega}:=\omega(\Gamma)=$ the period group of $(M, \omega)$.

\section{$\S 2$. Preliminary and review of the rational case}

\subsection{Novikov Floer cycles and spectral invariants}

Suppose that $\phi \in \operatorname{Ham}(M, \omega)$ is nondegenerate in the sense of Lefshetz fixed point theory: the derivative $T_{p} \phi: T_{p} M \rightarrow T_{p} M$ has no eigenvalue one at any fixed point $p \in M$. We will call a one-periodic Hamiltonian $H: S^{1} \times M \rightarrow \mathbb{R}$ nondegenerate if $\phi_{H}^{1}=\phi$ is a nondegenerate diffeomorphism. Note that the nondegeneracy of $H$ depends only on its time-one map $\phi_{H}^{1}$. We denote by $\operatorname{Per}(H)$ the set of contractible one-periodic orbits of $H$.

For each nondegenerate $H: S^{1} \times M \rightarrow \mathbb{R}$, we consider the free $\mathbb{Q}$ vector space over

$$
\operatorname{Crit} \mathcal{A}_{H}=\left\{[z, w] \in \widetilde{\Omega}_{0}(M) \mid z \in \operatorname{Per}(H)\right\} .
$$

Following $[8,10]$, we give the following definition.

Definition 2.1. We call the formal sum

$$
\beta=\sum_{[z, w] \in \operatorname{Crit} \mathcal{A}_{H}} a_{[z, w]}[z, w], a_{[z, w]} \in \mathbb{Q}
$$

a Novikov Floer chain (or simply a Floer chain) if there are only finitely many non-zero terms in the expression (2.2) above any given level of the action. We call $[z, w]$ a generator of the chain $\beta$ and denote

$$
[z, w] \in \beta
$$

if $a_{[z, w]} \neq 0$. We also say that $[z, w]$ contributes to $\beta$ in that case. We denote by $C F_{k}(H)$ the set of Floer chains whose generators all have degree $k$, i.e., satisfy

$$
\mu_{H}([z, w])=k,
$$

where $\mu_{H}$ is the Conley-Zehnder index of $[z, w][2]$.

Note that $C F_{*}(H)$ is a graded $\mathbb{Q}$-vector space. This is infinite dimensional as a $\mathbb{Q}$-vector space in general, unless $\pi_{2}(M)=0$.

Now we consider a Floer chain

$$
\beta=\sum a_{[z, w]}[z, w], \quad a_{[z, w]} \in \mathbb{Q} .
$$

The following notion plays an essential role for the mini-max argument via the Floer homology theory in $[15,18]$. 
Definition 2.2. Let $\beta$ be a Floer chain of a given degree $k$. We define the level of the cycle $\beta$ and denote by

$$
\lambda_{H}(\beta)=\max _{[z, w]}\left\{\mathcal{A}_{H}([z, w]) \mid a_{[z, w]} \neq 0 \text { in }(2.2)\right\}
$$

if $\beta \neq 0$, and just put $\lambda_{H}(0)=-\infty$ as usual. We call any element $[z, w]$ with $\mathcal{A}_{H}([z, w])=\lambda_{H}(\beta)$ a peak of $\beta$.

The level $\lambda_{H}$ induces a filtration of $C F_{*}(H)$ and so induces a natural nonArchimedean topology. (See [18, Appendix].) We regard each $C F_{k}(H)$ as a topological vector space with respect to this topology.

For a given one-periodic family $J=\left\{J_{t}\right\}_{0 \leq t \leq 1}$ of compatible almost complex structures, we consider the Floer boundary map

$$
\partial=\partial_{(J, H)}: C F_{*}(H) \rightarrow C F_{*}(H) .
$$

In Section 4, we will briefly review construction of $\partial$ in a way that is useful for our formulation of transversality problem of sub-homotopies. One can easily check that $\partial$ or all the natural operators arising in the Floer complex are continuous with respect to the above mentioned topology. We refer to [18, Appendix] for a precise description of the topology and for the proof of this continuity statements.

Definition 2.3. We say that a Floer chain $\beta \in C F(H)$ is a Floer cycle if $\partial \beta=0$ and a Floer boundary if $\beta=\partial \delta$ for a Floer chain $\delta$. Two Floer chains $\beta, \beta^{\prime}$ are said to be homologous if $\beta^{\prime}-\beta$ is a boundary.

Let

$$
a=\sum a_{A} q^{-A}, \quad a_{A} \in H^{*}(M)
$$

be a non-zero quantum cohomology class. We denote by $\Gamma(a) \subset \Gamma$ the set of $A$ 's for which the coefficient $a_{A}$ is non-zero. By the definition of the Novikov ring, we can enumerate $\Gamma(a)$ so that

$$
-\lambda_{1}<-\lambda_{2}<\cdots<-\lambda_{j}<\cdots,
$$

where $\lambda_{j}=\omega\left(A_{j}\right)$. We call the first term $a_{1} q^{-A_{1}}$ the leading order term of the quantum cohomology class $a$.

Next, for each given quantum cohomology class $0 \neq a \in Q H^{*}(M)$, we consider the Floer homology class $a^{b}$ dual to $a \in Q H^{*}(M)$. (See [17] for its precise meaning.) Then we associate the following mini-max value of the action functional $\mathcal{A}_{H}$

$$
\rho(H ; a)=\inf _{\alpha}\left\{\lambda_{H}(\alpha) \mid \alpha \in \operatorname{ker} \partial_{H} \subset C F_{n}(H) \text { with }[\alpha]=a^{b}\right\}
$$

to each given pair $(H, a)$. We like to emphasize that this definition itself manifests neither finiteness of the mini-max value nor its spectral property that $\rho(H ; a)$ is a critical value of $\mathcal{A}_{H}$. The finiteness was proved in [18] for a general symplectic manifold $(M, \omega)$ whether it is rational or not. However, we 
were able to prove the spectral property only for the rational case at the time of writing [18], which we now review.

\subsection{Review of the rational case}

We first recall an important notion of canonical thin cylinder between two nearby loops. We denote by $J_{\text {ref }}$ a fixed compatible almost complex structure and by exp the exponential map of the metric

$$
g:=\omega\left(\cdot, J_{\text {ref }} \cdot\right) .
$$

Let $\iota(g)$ be the injectivity radius of the metric $g$. As long as $d(x, y)<\iota(g)$ for the given two points of $M$, we can write

$$
y=\exp _{x}(\xi)
$$

for a unique vector $\xi \in T_{x} M$. As usual, we write the unique vector $\xi$ as

$$
\xi=\left(\exp _{x}\right)^{-1}(y) \text {. }
$$

Therefore if the $C^{0}$ distance $d_{C^{0}}\left(z, z^{\prime}\right)$ between the two loops

$$
z, z^{\prime}: S^{1} \rightarrow M
$$

is smaller than $\iota(g)$, we can define the canonical map

$$
u_{z z^{\prime}}^{c a n}:[0,1] \times S^{1} \rightarrow M
$$

by

$$
u_{z z^{\prime}}^{c a n}(s, t)=\exp _{z(t)}\left(\xi_{z z^{\prime}}(t)\right), \quad \text { or } \quad \xi_{z z^{\prime}}(t)=\left(\exp _{z(t)}\right)^{-1}\left(z^{\prime}(t)\right) .
$$

It is important to note that the image of $u_{z z^{\prime}}^{c a n}$ is contained in a small neighborhood of $z$ (or $z^{\prime}$ ), and uniformly converges to $z_{\infty}$ when $z$ and $z^{\prime}$ converge to a loop $z_{\infty}$ in the $C^{1}$ topology. Therefore $u_{z z^{\prime}}^{c a n}$ also picks out $\mathrm{s}$ a canonical homotopy class, denoted by $\left[u_{z z^{\prime}}^{c a n}\right]$, among the set of homotopy classes of the maps $u:[0,1] \times S^{1} \rightarrow M$ satisfying the given boundary condition

$$
u(0, t)=z(t), \quad u(1, t)=z^{\prime}(t) .
$$

The following lemma is an important ingredient in our proof, which will be used to overcome irrationality of symplectic manifolds later.

Lemma 2.4. Let $z, z^{\prime}: S^{1} \rightarrow M$ be two smooth loops and $u^{\text {can }}$ be the above

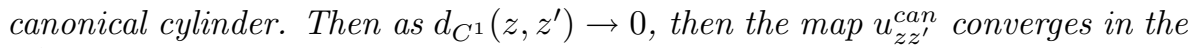
$C^{1}$-topology, and its geometric area Area $\left(u^{\text {can }}\right)$ converges to zero. In particular, we have the followings:

(1) For any bounding disc $w$ of $z$, the bounding disc

$$
w^{\prime}:=w \# u_{z z^{\prime}}^{c a n}
$$

of $w^{\prime}$ is pre-compact in the $C^{1}$-topology of the maps from the unit disc. 
(2)

$$
\int_{u_{z z^{\prime}}^{c a n}} \omega \rightarrow 0
$$

as $d_{C^{1}}\left(z, z^{\prime}\right) \rightarrow 0$ as $z^{\prime} \rightarrow z$.

Proof. (1) is an immediate consequence of the explicit form (2.6) of $u_{z z^{\prime}}^{c a n}$ and from the standard property of the exponential map.

On the other hand, from the explicit expression (2.6) of the canonical thin cylinder and from the property of the exponential map, it follows that the geometric area Area $\left(u_{i \infty}^{c a n}\right)$ converges to zero as $d_{C^{1}}\left(z, z^{\prime}\right) \rightarrow 0$ by an easy area estimate. Since $z, z^{\prime}$ are assumed to be $C^{1}$, it follows $u_{z z^{\prime}}^{c a n}$ is $C^{1}$ and hence the inequality

$$
\operatorname{Area}\left(u_{i \infty}^{c a n}\right) \geq\left|\int_{u_{i \infty}^{c a n}} \omega\right|
$$

This implies

$$
\lim _{j \rightarrow \infty} \int_{u_{i \infty}^{c a n}} \omega=0
$$

which finishes the proof.

The following theorem was previously proved by the author in [18]. We duplicate its proof here to highlight differences between the rational and the irrational cases, and to motivate the scheme of our proof in the irrational case.

Theorem 2.5 ([18, Theorem 7.1]). Suppose that $(M, \omega)$ is rational. Then for any smooth one-periodic Hamiltonian function $H: S^{1} \times M \rightarrow \mathbb{R}$, we have

$$
\rho(H ; a) \in \operatorname{Spec}(H)
$$

for each given quantum cohomology class $0 \neq a \in Q H^{*}(M)$.

Proof. We need to show that the mini-max value $\rho(H ; a)$ is a critical value, or that there exists $[z, w] \in \widetilde{\Omega}_{0}(M)$ such that

$$
\begin{aligned}
& \mathcal{A}_{H}([z, w])=\rho(H ; a) \\
& d \mathcal{A}_{H}([z, w])=0, \quad \text { i.e., } \quad \dot{z}=X_{H}(z) .
\end{aligned}
$$

The finiteness of the value $\rho(H ; a)$ was proved in [18]. If $H$ is nondegenerate, we just use the fixed Hamiltonian $H$. If $H$ is degenerate, we approximate $H$ by a sequence of nondegenerate Hamiltonians $H_{i}$ in the $C^{2}$ topology. Let $\left[z_{i}, w_{i}\right] \in \operatorname{Crit} \mathcal{A}_{H_{i}}$ be a peak of the Floer cycle $\alpha_{i} \in C F_{*}\left(H_{i}\right)$, such that

$$
\lim _{j \rightarrow \infty} \mathcal{A}_{H_{i}}\left(\left[z_{i}, w_{i}\right]\right)=\rho(H ; a) .
$$

Such a sequence can be chosen by the definition of $\rho(\cdot ; a)$ and its finiteness property. 
Since $M$ is compact and $H_{i} \rightarrow H$ in the $C^{2}$ topology, and $\dot{z}_{i}=X_{H_{i}}\left(z_{i}\right)$ for all $i$, it follows from the standard boot-strap argument that $z_{i}$ has a subsequence, which we still denote by $z_{i}$, converging to some loop $z_{\infty}: S^{1} \rightarrow M$ satisfying $\dot{z}=X_{H}(z)$. Now we show that the sequence $\left[z_{i}, w_{i}\right]$ are pre-compact on $\widetilde{\Omega}_{0}(M)$. Since we fix the quantum cohomology class $0 \neq a \in Q H^{*}(M)$ (or more specifically since we fix its degree) and since the Floer cycle is assumed to satisfy $\left[\alpha_{i}\right]=a^{b}$, we have

$$
\mu_{H_{i}}\left(\left[z_{i}, w_{i}\right]\right)=\mu_{H_{j}}\left(\left[z_{j}, w_{j}\right]\right) .
$$

Lemma 2.6. When $(M, \omega)$ is rational, Crit $\mathcal{A}_{K} \subset \widetilde{\Omega}_{0}(M)$ is a closed subset of $\mathbb{R}$ for any smooth Hamiltonian $K$, and is locally compact in the subspace topology of the covering space

$$
\pi: \widetilde{\Omega}_{0}(M) \rightarrow \Omega_{0}(M)
$$

Proof. First note that when $(M, \omega)$ is rational, the covering group $\Gamma$ of $\pi$ above is discrete. Together with the fact that the set of solutions of $\dot{z}=X_{K}(z)$ is compact (on compact $\mathrm{M}$ ), it follows that

$$
\operatorname{Crit}\left(\mathcal{A}_{K}\right)=\left\{[z, w] \in \widetilde{\Omega}_{0}(M) \mid \dot{z}=X_{K}(z)\right\}
$$

is a closed subset which is also locally compact.

Now consider the bounding discs of $z_{\infty}$ given by

$$
w_{i}^{\prime}=w_{i} \# u_{i \infty}^{c a n}
$$

for all sufficiently large $i$, where $u_{i \infty}^{c a n}=u_{z_{i} z_{\infty}}^{c a n}$ is the canonical thin cylinder between $z_{i}$ and $z_{\infty}$. We note that as $i \rightarrow \infty$ the geometric area of $u_{i \infty}^{c a n}$ converges to 0 .

We compute the action of the critical points $\left[z_{\infty}, w_{i}^{\prime}\right] \in \operatorname{Crit} \mathcal{A}_{H}$,

$$
\begin{aligned}
& \mathcal{A}_{H}\left(\left[z_{\infty}, w_{i}^{\prime}\right]\right)=-\int_{w_{i}^{\prime}} \omega-\int_{0}^{1} H\left(t, z_{\infty}(t)\right) d t \\
&=-\int_{w_{i}} \omega-\int_{u_{i \infty}^{\text {can }}}-\int_{0}^{1} H\left(t, z_{\infty}(t)\right) d t \\
&=\left(-\int_{w_{i}} \omega-\int_{0}^{1} H_{i}\left(t, z_{i}(t)\right) d t\right) \\
&-\left(\int_{0}^{1} H\left(t, z_{\infty}(t)\right)-\int_{0}^{1} H_{i}\left(t, z_{i}(t)\right)\right)-\int_{u_{i \infty}^{\text {can }}} \omega \\
&=\mathcal{A}_{H_{i}}\left(\left[z_{i}, w_{i}\right]\right)-\left(\int_{0}^{1} H\left(t, z_{\infty}(t)\right)-\int_{0}^{1} H_{i}\left(t, z_{i}(t)\right)\right) \\
&-\int_{u_{i \infty}^{\text {can }}} \omega .
\end{aligned}
$$


Since $z_{i}$ converges to $z_{\infty}$ uniformly and $H_{i} \rightarrow H$, we have

$$
-\left(\int_{0}^{1} H\left(t, z_{\infty}(t)\right)-\int_{0}^{1} H\left(t, z_{i}(t)\right)\right) \rightarrow 0 .
$$

Therefore combining (2.7), (2.8) and (2.11), we derive

$$
\lim _{i \rightarrow \infty} \mathcal{A}_{H}\left(\left[z_{\infty}, w_{i}^{\prime}\right]\right)=\rho(H ; a) .
$$

In particular $\mathcal{A}_{H}\left(\left[z_{\infty}, w_{i}^{\prime}\right]\right)$ is a Cauchy sequence, which implies

$$
\left|\int_{w_{i}^{\prime}} \omega-\int_{w_{j}^{\prime}} \omega\right|=\left|\mathcal{A}_{H}\left(\left[z_{\infty}, w_{i}^{\prime}\right]\right)-\mathcal{A}_{H}\left(\left[z_{\infty}, w_{j}^{\prime}\right]\right)\right| \rightarrow 0
$$

i.e.,

$$
\int_{w_{i}^{\prime} \# \bar{w}_{j}^{\prime}} \omega \rightarrow 0
$$

Since $\Gamma$ is discrete and $\int_{w_{i}^{\prime} \# \bar{w}_{j}^{\prime}} \omega \in \Gamma$, this indeed implies that

$$
\int_{w_{i}^{\prime} \# \bar{w}_{j}^{\prime}} \omega=0
$$

for all sufficiently large $i, j \in \mathbb{Z}_{+}$. Since the set $\left\{\int_{w_{i}^{\prime}} \omega\right\}_{i \in \mathbb{Z}_{+}}$is bounded, we conclude that the sequence $\int_{w_{i}^{\prime}} \omega$ eventually stabilize, by choosing a subsequence if necessary. Going back to (2.9), we derive that the actions

$$
\mathcal{A}_{H}\left(\left[z_{\infty}, w_{i}^{\prime}\right]\right)
$$

themselves stabilize and so we have

$$
\mathcal{A}_{H}\left(\left[z_{\infty}, w_{N}^{\prime}\right]\right)=\lim _{i \rightarrow \infty} \mathcal{A}_{H}\left(\left[z_{\infty}, w_{i}^{\prime}\right]\right)=\rho(H ; a)
$$

for a fixed sufficiently large $N \in \mathbb{Z}_{+}$. This proves that $\rho(H ; a)$ is indeed the value of $\mathcal{A}_{H}$ at the critical point $\left[z_{\infty}, w_{N}^{\prime}\right]$. This finishes the proof.

In fact, an examination of the above proof proves a stronger fact that the mini-maxing sequence $\left[z_{i}, w_{i}\right]$ is precompact for the rational case, which we now explain. We recall that if $H, H^{\prime}$ are nondegenerate and sufficiently $C^{2}$-close, there exists a canonical one-one correspondence between the set of associated Hamiltonian periodic orbits. We call an associated pair any pair $\left(z, z^{\prime}\right)$ of Hamiltonian periodic orbits of $H, H^{\prime}$ mapped to each other under this correspondence. We will give the proof of the following proposition in the Appendix.

Proposition 2.7. Suppose that $H, H^{\prime}$ are nondegenerate and sufficiently $C^{2}$ close. Let $\left(z, z^{\prime}\right)$ be an associated pair of $H, H^{\prime}$. Then we have

$$
\mu_{H}([z, w])=\mu_{H^{\prime}}\left(\left[z^{\prime}, w \# u_{z z^{\prime}}^{c a n}\right]\right) .
$$


We derive

$$
\begin{aligned}
2 c_{1}\left(\left[w_{i}^{\prime} \# \bar{w}_{j}^{\prime}\right]\right) & =2 c_{1}\left(\left[w_{i} \# u_{i \infty}^{c a n} \# \overline{w_{j} \# u_{j \infty}^{c a n}}\right]\right) \\
& =2 c_{1}\left(\left[w_{i} \# u_{i \infty}^{c a n} \# \bar{u}_{j \infty}^{c a n} \# \bar{w}_{j}\right]\right) \\
& =\mu_{H_{i}}\left(\left[z_{i}, w_{i}\right]\right)-\mu_{H_{i}}\left(\left[z_{i}, w_{j} \# u_{j \infty}^{c a n} \# \bar{u}_{i \infty}^{c a n}\right]\right) .
\end{aligned}
$$

The third equality comes from the index formula

$$
\mu_{H}([z, w \# A])=\mu_{H}([z, w])-2 c_{1}(A)
$$

(see [18, Appendix] for the details of its proof). On the other hand, we derive

$$
\mu_{H_{i}}\left(\left[z_{i}, w_{j} \# u_{j \infty}^{c a n} \# \bar{u}_{i \infty}^{c a n}\right]\right)=\mu_{H_{i}}\left(\left[z_{i}, w_{j} \# u_{z_{j} z_{i}^{\prime}}^{c a n}\right]\right)=\mu_{H_{j}}\left(\left[z_{j}, w_{j}\right]\right)
$$

when $i, j$ are sufficiently large. Here the first equality follows since $u_{i \infty}^{c a n} \# \bar{u}_{i \infty}^{c a n}$ is homotopic to the canonical thin cylinder $u_{z_{j} z_{i}^{\prime}}^{c a n}$, and the second comes from (2.13). On the other hand, $\left[z_{i}, w_{i}\right]$ and $\left[z_{j}, w_{j}\right]$ satisfy

$$
\mu_{H_{i}}\left(\left[z_{i}, w_{i}\right]\right)=\mu_{H_{j}}\left(\left[z_{j}, w_{j}\right]\right)
$$

because they are generators of Floer cycles $\alpha_{i}$ and $\alpha_{j}$ both representing the same Floer homology class $a^{b}$ and so having the same degree. Hence combining (2.14)-(2.16), we obtain

$$
c_{1}\left(\left[w_{i}^{\prime} \# \bar{w}_{j}^{\prime}\right]\right)=0
$$

for all sufficiently large $i, j$. Combining (2.12) and (2.17), we have proved

$$
\left[z_{\infty}, w_{i}^{\prime}\right]=\left[z_{\infty}, w_{j}^{\prime}\right] \text { in } \widetilde{\Omega}_{0}(M) .
$$

If we denote by $\left[z_{\infty}, w_{\infty}\right]$ this common element of $\widetilde{\Omega}_{0}(M)$, we have proven that the sequence $\left[z_{i}, w_{i}\right]$ converges to a critical point $\left[z_{\infty}, w_{\infty}\right]$ of $\mathcal{A}_{H}$ in the topology of the covering space $\pi: \widetilde{\Omega}_{0}(M) \rightarrow \Omega_{0}(M)$. This finishes our discussion about the rational case.

For the irrational case, the above argument breaks down since the sequence $\left[z_{\infty}, w_{i}^{\prime}\right]$ used in the above proof will not stabilize, and more seriously the action values $\mathcal{A}_{H}\left(\left[z_{\infty}, w_{i}^{\prime}\right]\right)$ may accumulate at a value in $\mathbb{R} \backslash \operatorname{Spec}(H)$. Recall that in the irrational case, $\operatorname{Spec}(H)$ is a dense subset of $\mathbb{R}$. Therefore in the irrational case, one needs to directly prove that the sequence has a convergent subsequence in the natural topology of $\widetilde{\Omega}_{0}(M)$. It turns out that the above limiting arguments used for the rational case cannot be carried out due to the possibility that the discs $w_{i}$ could behave wildly in the limiting process. As a result, proving such a convergence is not possible in general even for the nondegenerate case for a given mini-max sequence of critical points $\left[z_{i}, w_{i}\right]$ satisfying (2.8). One needs to use a mini-max sequence of cycles instead. This scheme is exactly what we have carried out in the present paper. Because we use the Floer cycles and they are defined only for nondegenerate Hamiltonians, we can prove the spectrality axiom only for the nondegenerate case in this way. 
Along the way, we develop many new ingredients in the chain level Floer theory needed to carry out the scheme. We call our chain level theory the Floer mini-max theory.

To go to the case of degenerate Hamiltonians, it is unavoidable to use the approximation used arguments above as in the rational case. It would be very interesting to see if this difficulty is something intrinsic for this case. (See Remark 9.4 for some related comments.)

\section{§3. The Cerf bifurcation diagram}

\subsection{Generic bifurcations of the critical set of the action functional}

We first recall that for a generic one-parameter family $\mathcal{H}=\{H(\eta)\}_{0 \leq \eta \leq 1}$, there are a finite number of points

$$
\operatorname{Sing}(\mathcal{H})=\left\{s_{1}, s_{2}, \ldots, s_{k_{1}}\right\} \subset[0,1],
$$

where there occurs either birth-death or death-birth type of bifurcation of periodic orbits (see [11] for a detailed proof of this). Furthermore at each such $s_{i}$, there is exactly one-periodic orbit $z_{i}$ of $\dot{x}=X_{H\left(s_{i}\right)}(x)$ for which a continuous family of the pair $z^{+}(\eta), z^{-}(\eta)$ of periodic orbits of $\dot{x}=X_{H(\eta)}(x)$ bifurcate from $z_{i}$ for $\eta$ with $\left|\eta-s_{i}\right|<\delta, \delta$ sufficiently small, that satisfy

(1) $z^{ \pm}(\eta) \rightarrow z_{i}$ as $\eta \rightarrow s_{i}$

(2) the Conley-Zehnder indices satisfy

$$
\mu\left(\left[z^{+}, w^{+}\right]\right)=\mu\left(\left[z^{-}, w^{-}\right]\right)+1,
$$

where $w^{+} \sim w^{-} \# u$ for $u$ the thin cylinder between $z^{+}$and $z^{-}$. This latter condition makes sense because $z^{+}$and $z^{-}$are close when $\delta$ is sufficiently small, which depends only on the homotopy $\mathcal{H}$ independent of points $s_{i} \in \operatorname{Sing}(\mathcal{H})$. We denote this uniform $\delta$ as

$$
\delta_{1}=\delta_{1}(\mathcal{H}) .
$$

In the course of studying a detailed structure of the Cerf-type bifurcation diagram of the action functional, we will provide an outline of a proof of the above statements for the reader's convenience leaving more details to [11].

Let $\mathcal{H}=\{H(\eta)\}_{0 \leq \eta \leq 1}$ be a homotopy (or a one-parameter family) of smooth Hamiltonians. We denote

$$
\operatorname{Crit} \mathcal{A}_{\mathcal{H}}:=\bigcup_{\eta \in[0,1]}\left\{[z, w] \mid \dot{z}=X_{H(\eta)}(z)\right\}=\bigcup_{\eta \in[0,1]} \operatorname{Crit} \mathcal{A}_{H(\eta)}
$$

and consider it as a subset of $[0,1] \times \widetilde{\Omega}_{0}(M)$. For the simplicity of notations, we sometimes denote

$$
\mathcal{H}_{m}(M):=C_{m}^{\infty}\left(S^{1} \times M\right)
$$

We then define

$$
\mathcal{P} \mathcal{H}_{m}(M):=C^{\infty}\left([0,1], \mathcal{H}_{m}(M)\right)
$$


and

$$
\mathcal{P}\left(\mathcal{H}_{m}(M) ; H_{0}, H_{1}\right):=\left\{\mathcal{H} \in \mathcal{P} \mathcal{H}_{m}(M) \mid H(0)=H_{0}, H(1)=H_{1}\right\}
$$

for any given Hamiltonians $H_{1}, H_{2}$. We also denote

$$
\mathcal{H}_{m}^{n d}(M):=\left\{H \in \mathcal{H}_{m}(M) \mid H \quad \text { is nondegenerate }\right\} .
$$

The following lemma is easy to prove from the definition and from the standard facts on the first order ordinary differential equation.

Lemma 3.1. Let $H_{\alpha}, H_{\beta}$ be smooth and $\mathcal{H} \in \mathcal{P}\left(\mathcal{H}_{m}(M) ; H_{\alpha}, H_{\beta}\right)$. Then we have

(1) Crit $\mathcal{A}_{\mathcal{H}}$ is invariant under the deck transformation of $\Gamma$ on $\widetilde{\Omega}_{0}(M) \times$ $[0,1]$.

(2) Under the covering map $\pi: \widetilde{\Omega}_{0}(M) \rightarrow \Omega_{0}(M)$, Crit $\mathcal{A}_{\mathcal{H}} / \Gamma$ coincides with

$$
\operatorname{Per}(\mathcal{H}):=\bigcup_{\eta \in[0,1]} \operatorname{Per}(H(\eta)) \subset[0,1] \times \Omega_{0}(M)
$$

and in particular is compact.

Now we prove the following general structure theorem on $\operatorname{Crit} \mathcal{A}_{\mathcal{H}}$ for a generic homotopy $\mathcal{H}$ such that the end points $H(0)$ and $H(1)$ are nondegenerate.

Proposition 3.2. Let $H_{\alpha}, H_{\beta}$ be two nondegenerate Hamiltonian functions and $\mathcal{H} \in \mathcal{P}\left(\mathcal{H}_{m}(M) ; H_{\alpha}, H_{\beta}\right)$. Then there exists a dense subset

$$
\mathcal{P}^{\text {reg }}\left(\mathcal{H}_{m}(M) ; H_{\alpha}, H_{\beta}\right) \subset \mathcal{P}\left(\mathcal{H}_{m}(M) ; H_{0}, H_{1}\right)
$$

such that the subset

$$
\operatorname{Crit} \mathcal{A}_{\mathcal{H}} \subset[0,1] \times \widetilde{\Omega}_{0}(M)
$$

becomes a smooth one-manifold with its boundary $\partial\left(\right.$ Crit $\left.\mathcal{A}_{\mathcal{H}}\right)$ contained in $\{0,1\}$ $\times \widetilde{\Omega}_{0}(M)$.

Proof. We first note that the action by $\Gamma$ on $\operatorname{Crit} \mathcal{A}_{\mathcal{H}}$ is free. Therefore it is enough to prove that $\operatorname{Crit} \mathcal{A}_{\mathcal{H}} / \Gamma$ is a smooth one-manifold for some generic choices of $\mathcal{H}$. We also know

$$
\operatorname{Crit} \mathcal{A}_{\mathcal{H}} / \Gamma=\operatorname{Per}(\mathcal{H}) \text {. }
$$

This in turn implies that it is enough to prove that $\operatorname{Per}(\mathcal{H})$ becomes a $($ compact) smooth one-manifold for some generic choices of $\mathcal{H}$. We now prove this statement.

We first remark that in the Fredholm analysis we are going to carry out below, one needs to take a suitable Banach completion of the various function spaces that appear. However this is a standard procedure by now, and so we will not mention this technicality but just work with $C^{\infty}$ function spaces. A good reference for this matter and also for detailed calculations involving the action functional is the paper [24] by Weinstein. 
We define the map

$$
\Phi: \Omega_{0}(M) \times \mathcal{P}\left(\mathcal{H}_{m}(M) ; H_{\alpha}, H_{\beta}\right) \times[0,1] \rightarrow T \Omega_{0}(M) \times[0,1]
$$

by

$$
\Phi(z, \mathcal{H}, \eta) \mapsto\left(\dot{z}-X_{H(\eta)}(z), \eta\right) .
$$

Considering this as the composition of the section

$$
(z, \mathcal{H}, \eta) \rightarrow\left(\dot{z}-X_{H(\eta)}(z), \mathcal{H}, \eta\right)
$$

of the parametric tangent bundle

$T \Omega_{0}(M) \times \mathcal{P}\left(\mathcal{H}_{m}(M) ; H_{\alpha}, H_{\beta}\right) \times[0,1] \rightarrow \Omega_{0}(M) \times \mathcal{P}\left(\mathcal{H}_{m}(M) ; H_{\alpha}, H_{\beta}\right) \times[0,1]$

and the projection map

$$
\Omega_{0}(M) \times \mathcal{P}\left(\mathcal{H}_{m}(M) ; H_{\alpha}, H_{\beta}\right) \times[0,1] \rightarrow \Omega_{0}(M) \times[0,1],
$$

it is straightforward to check that the derivative of $\Phi$ is surjective at all the zero points $(z, \mathcal{H}, \eta)$ of $\Phi$, i.e., those satisfying $\dot{z}=X_{H(\eta)}(z)$. Therefore the universal set of periodic orbits, denoted by

$$
\mathcal{P} e r:=\Phi^{-1}\left(o_{T \Omega_{0}(M)} \times \mathcal{P}\left(\mathcal{H}_{m}(M) ; H_{\alpha}, H_{\beta}\right) \times[0,1]\right),
$$

is a smooth submanifold of $\Omega_{0}(M) \times \mathcal{P}\left(\mathcal{H}_{m}(M) ; H_{\alpha}, H_{\beta}\right) \times[0,1]$ by the implicit function theorem.

Furthermore it is well-known that the linearization map

$$
\xi \mapsto \frac{D \xi}{d t}-D X_{K}(z)(\xi)
$$

along the periodic orbit $z$ of a Hamiltonian $K \in \mathcal{H}_{m}(M)$ is a Fredholm operator of index zero after making a suitable Banach completion of $\Omega_{0}(M)$ (see [24] for example). This then is translated into the statement that the projection map $(3.12) \pi_{2}: \mathcal{P} e r \subset \Omega_{0}(M) \times \mathcal{P}\left(\mathcal{H}_{m}(M) ; H_{\alpha}, H_{\beta}\right) \times[0,1] \rightarrow \mathcal{P}\left(\mathcal{H}_{m}(M) ; H_{\alpha}, H_{\beta}\right)$ is a Fredholm map of index 1 . Now by the Sard-Smale theorem, the set of regular values of $\pi_{2}$, which we denote by

$$
\mathcal{P}^{r e g}\left(\mathcal{H}_{m}(M) ; H_{\alpha}, H_{\beta}\right)
$$

is residual, and in particular dense. This finishes the proof.

Next a simple version of the two-jet transversality implies the following, whose proof we omit and refer to [11] for more details.

Proposition 3.3. Let $\mathcal{H} \in \mathcal{P}^{\text {reg }}\left(\mathcal{H}_{m}(M) ; H_{1}, H_{2}\right)$. Then the set $\operatorname{Sing}(\mathcal{H}):=\{\eta \in[0,1] \mid$ the linearization map (3.11) is not surjective for $H(\eta)\}$ is finite. And there is another smaller dense subset of

$$
\mathcal{H} \in \mathcal{P}^{r e g}\left(\mathcal{H}_{m}(M) ; H_{1}, H_{2}\right)
$$

for which at each point $\eta \in \mathcal{S}$ ing $(\mathcal{H})$, either birth-death or death-birth type of bifurcation occurs as described in the beginning of this section. 


\subsection{The Cerf bifurcation diagram}

Next we introduce a notion of the Cerf bifurcation diagram of the action functionals and study its structure for a generic choice of the homotopy $\mathcal{H}$.

Definition 3.4. Consider the set

$$
\begin{aligned}
\Sigma(\mathcal{H})= & \left\{(\eta, a) \mid \eta \in[0,1], a=\mathcal{A}_{H(\eta)}([z, w]),[z, w] \in \operatorname{Crit} \mathcal{A}_{H(\eta)}\right\} \\
& \subset[0,1] \times \mathbb{R} .
\end{aligned}
$$

We call $\Sigma(\mathcal{H})$ the Cerf bifurcation diagram of the homotopy $\mathcal{H}$.

There is the natural evaluation homomorphism

$$
g \in \Gamma \mapsto \omega(g) \in \Gamma_{\omega} \subset \mathbb{R} .
$$

Via the homomorphism (3.14), $\Gamma$ naturally acts on $[0,1] \times \mathbb{R}$ by

$$
\Gamma \times[0,1] \times \mathbb{R} \rightarrow[0,1] \times \mathbb{R} ; \quad g \cdot(\eta, a) \mapsto(\eta, a-\omega(g))
$$

which preserves $\Sigma(\mathcal{H})$. Now we consider the map $\widetilde{\Phi}$

$$
\begin{aligned}
\widetilde{\Phi}: \widetilde{\Omega}_{0}(M) & \times \mathcal{P}\left(\mathcal{H}_{m}(M) ; H_{\alpha}, H_{\beta}\right) \times[0,1] \\
& \rightarrow \widetilde{T} \Omega_{0}(M) \times \mathcal{P}\left(\mathcal{H}_{m}(M) ; H_{\alpha}, H_{\beta}\right) \times[0,1] \times \mathbb{R}
\end{aligned}
$$

defined by

$$
\widetilde{\Phi}([z, w], \mathcal{H}, \eta)=\left(\dot{z}-X_{H(\eta)}(z), \mathcal{H}, \eta, \mathcal{A}_{H(\eta)}([z, w])\right) .
$$

This map is equivariant under the obvious actions of $\Gamma$.

It follows from Proposition 3.2 that $\widetilde{\Phi}$ is transverse to the submanifold

$$
o_{T \Omega_{0}(M)} \times[0,1] \times \mathbb{R} \subset T \Omega_{0}(M) \times[0,1] \times \mathbb{R} .
$$

In particular, we know that

$$
\mathcal{Z}:=(\widetilde{\Phi})^{-1}\left(o_{T \Omega_{0}(M)} \times[0,1] \times \mathbb{R}\right)
$$

is a smooth submanifold of

$$
\widetilde{\Omega}_{0}(M) \times \mathcal{P}\left(\mathcal{H}_{m}(M) ; H_{\alpha}, H_{\beta}\right) \times[0,1] .
$$

Obviously we have the natural (étale) covering map

$$
\Gamma \rightarrow \mathcal{Z} \rightarrow \mathcal{P} e r=\bigcup_{\mathcal{H} \in \mathcal{P}\left(\mathcal{H}_{m}(M) ; H_{\alpha}, H_{\beta}\right)} \operatorname{Per}(\mathcal{H})
$$

Now we consider the projection

$$
\Pi: \mathcal{Z} \subset \widetilde{\Upsilon} \rightarrow \mathcal{P}\left(\mathcal{H}_{m}(M) ; H_{\alpha}, H_{\beta}\right)
$$

It is easy to check that this is a Fredholm map of index 1. By the Sard-Smale theorem, there is another dense subset set of $\mathcal{H}$ 's such that

$$
\Pi^{-1}(\mathcal{H})=: Z(\mathcal{H})
$$

becomes a smooth one dimensional manifold with boundary

$$
\partial Z(\mathcal{H})=\Psi^{-1}\left(o_{T \Omega_{0}(M)} \times\{0,1\} \times \mathbb{R}\right),
$$


as long as it is non-empty. In addition, $\Gamma$ freely acts on $Z(\mathcal{H})$ and hence comes a natural $\Gamma$ principal bundle

$$
\pi: Z(\mathcal{H}) \rightarrow Z(\mathcal{H}) / \Gamma
$$

where the quotient can be canonically identified with $\operatorname{Per}(\mathcal{H})$. By the standard a priori estimates on the Hamilton equation, $\operatorname{Per}(\mathcal{H})$ is shown to be a compact one manifold with boundary.

Now consider the map

$$
\text { ev }: Z(\mathcal{H}) \subset \widetilde{\Omega}_{0}(M) \times[0,1] \rightarrow[0,1] \times \mathbb{R}
$$

defined by

$$
([z, w], \eta) \rightarrow\left(\eta, \mathcal{A}_{H(\eta)}([z, w])\right)
$$

for which the diagram

$$
\begin{array}{ccc}
Z(\mathcal{H}) & \longrightarrow & {[0,1] \times \mathbb{R}} \\
\downarrow & & \downarrow \\
{[0,1]} & \equiv & {[0,1]}
\end{array}
$$

commutes. Furthermore $e v$ is equivariant under the fiber-preserving action of $\Gamma$, and the Cerf diagram $\Sigma(\mathcal{H})$ is nothing but the image of $e v$. Hence there induces a natural action of $\Gamma$ acting fiberwise on $\Sigma(\mathcal{H})$ under the projection $\Sigma(\mathcal{H}) \rightarrow[0,1]$

Now we describe structure of the image of the map (3.20). We first prove the following lemma, which states that the action cannot be the same for two different critical points $\left[z_{1}, w_{1}\right]$ and $\left[z_{2}, w_{2}\right]$ of the form $z_{1}=z_{2}$, if we further require $\mu_{K}\left(\left[z_{1}, w_{1}\right]\right)=\mu_{K}\left(\left[z_{2}, w_{2}\right]\right)$.

Lemma 3.5. Let $K$ be a nondegenerate Hamiltonian function and consider the elements from $\operatorname{Crit}\left(\mathcal{A}_{K}\right)$. Then for any element $z \in \operatorname{Per}(K), \mathcal{A}_{K}\left(\left[z, w_{1}\right]\right)=$ $\mathcal{A}_{K}\left(\left[z, w_{2}\right]\right)$ and $\mu_{K}\left(\left[z, w_{1}\right]\right)=\mu_{K}\left(\left[z, w_{2}\right]\right)$ if and only if $\left[z, w_{1}\right]=\left[z, w_{2}\right]$. In particular, if we have

$$
\mathcal{A}_{K}\left(\left[z_{1}, w_{1}\right]\right)=\mathcal{A}_{K}\left(\left[z_{2}, w_{2}\right]\right), \quad \mu_{K}\left(\left[z_{1}, w_{1}\right]\right)=\mu_{K}\left(\left[z_{2}, w_{2}\right]\right),
$$

then $z_{1} \neq z_{2}$ unless $\left[z_{1}, w_{1}\right]=\left[z_{2}, w_{2}\right]$.

Proof. Suppose

$$
\mu_{K}\left(\left[z, w_{1}\right]\right)=\mu_{K}\left(\left[z, w_{2}\right]\right)
$$

and

$$
\mathcal{A}_{K}\left(\left[z, w_{1}\right]\right)=\mathcal{A}_{K}\left(\left[z, w_{2}\right]\right)
$$

From the assumption (3.22), we derive

$$
\int_{w_{2} \# \bar{w}_{1}} \omega=0
$$

since we have

$$
\mathcal{A}_{K}\left(\left[z, w_{1}\right]\right)-\mathcal{A}_{K}\left(\left[z, w_{2}\right]\right)=\int_{w_{1} \# \bar{w}_{2}} \omega .
$$


And from (3.21) and the index formula

$$
\mu_{K}\left(\left[z, w_{1}\right]\right)=\mu_{K}\left(\left[z, w_{2}\right]\right)-2 c_{1}\left(w_{1} \# \bar{w}_{2}\right)
$$

we derive

$$
c_{1}\left(w_{1} \# \bar{w}_{2}\right)=0 .
$$

Therefore (3.23) and (3.24) imply $\left[z, w_{1}\right]=\left[z, w_{2}\right]$ by the definition of $\Gamma$ equivalence classes in (1.1). The converse is obvious.

Definition 3.6. Let $H_{1}, H_{2}$ be two nondegenerate Hamiltonians and $\mathcal{H}$ be a homotopy between them. Let $\Sigma(\mathcal{H}) \subset[0,1] \times \mathbb{R}$ be the associated Cerf diagram.

(1) We say that a point $(\eta, a) \in \Sigma(\mathcal{H})$ is a cusp if $\eta \in \operatorname{Sing}(\mathcal{H})$ and $\mathcal{A}_{H(\eta)}([z, w])=a$, and a generic cusp if the second derivative

$$
d^{2} \mathcal{A}_{H(\eta)}([z, w])
$$

at $[z, w]$ has exactly one-dimensional kernel.

(2) We say that a point $(\eta, a) \in \Sigma(\mathcal{H})$ is a crossing if there are two different $\left[z_{1}, w_{1}\right],\left[z_{2}, w_{2}\right] \in \operatorname{Crit} \mathcal{A}_{H(\eta)}$ with

$$
\begin{aligned}
a=\mathcal{A}_{H(\eta)}\left(\left[z_{1}, w_{1}\right]\right) & =\mathcal{A}_{H(\eta)}\left(\left[z_{2}, w_{2}\right]\right) \\
\mu_{H(\eta)}\left(\left[z_{1}, w_{1}\right]\right) & =\mu_{H(\eta)}\left(\left[z_{2}, w_{2}\right]\right)
\end{aligned}
$$

and a nondegenerate crossing if it has the property that the corresponding branches intersect transversely. We denote by

$$
\operatorname{Cross}^{n d}(\mathcal{H}) \subset[0,1]
$$

the set of nondegenerate crossings. If there are exactly one such nondegenerate crossing at $\eta$, modulo the action of $\Gamma$, whose associated pair of critical points $\left[z_{i}, w_{i}\right], i=1,2$ in addition, we call the crossing a generic crossing.

We note that due to the action of $\Gamma$ on the Cerf diagram, the set $\operatorname{Cross}^{n d}(\mathcal{H})$ is a countable infinite subset of $[0,1]$. We like to emphasize that $\operatorname{Cross}^{n d}(\mathcal{H})$ does not include the points $(\eta, a)$ with $a=\mathcal{A}_{H(\eta)}\left(\left[z_{1}, w_{1}\right]\right)=\mathcal{A}_{H(\eta)}\left(\left[z_{2}, w_{2}\right]\right)$ with different Conley-Zehnder indices. There exists a natural fiberwise action of the group $\Gamma$ on $\Sigma(\mathcal{H})$ under the projection

$$
\pi_{1}: \Sigma(\mathcal{H}) \subset[0,1] \times \mathbb{R} \rightarrow[0,1] .
$$

With these definitions, we prove the following structure theorem of the Cerf bifurcation diagram for a generic homotopy $\mathcal{H}$.

Theorem 3.7. Let $H_{1}, H_{2}$ be two nondegenerate Hamiltonians. Then there exists a dense subset

$$
\mathcal{P}^{\operatorname{Cerf}}\left(\mathcal{H}_{m}(M) ; H_{0}, H_{1}\right) \subset \mathcal{P}^{r e g}\left(\mathcal{H}_{m}(M) ; H_{0}, H_{1}\right) \subset \mathcal{P}\left(\mathcal{H}_{m}(M) ; H_{0}, H_{1}\right)
$$

such that for any element $\mathcal{H} \in \mathcal{P}^{\operatorname{Cerf}}\left(\mathcal{H}_{m}(M) ; H_{0}, H_{1}\right)$ its associated Cerf diagram $\Sigma(\mathcal{H})$ satisfies the following list of the properties: 
(1) $\Sigma(\mathcal{H})$ is the projection of the one-manifold $Z(\mathcal{H})$, with boundary contained in $\pi_{1}^{-1}\left(\widetilde{\Omega}_{0}(M) \times\{0,1\} \times \mathbb{R}\right)$.

(2) All the crossings are nondegenerate and unique modulo the action of $\Gamma$.

(3) There is exactly one cusp point $(\eta, a)$ unique, modulo the action of $\Gamma$, corresponding to each point $\eta \in \mathcal{S} \operatorname{Sing}(\mathcal{H})$.

(4) $\operatorname{Sing}(\mathcal{H}) \cap \mathcal{C r o s s}^{\text {nd }}(\mathcal{H})=\emptyset$.

Proof. We denote the diagonal subset of $\Omega_{0}(M) \times \Omega_{0}(M)$ by $\Delta$, and let

$$
\widetilde{\Delta}:=\pi^{-1}(\Delta) \subset \widetilde{\Omega}_{0}(M) \times \widetilde{\Omega}_{0}(M)
$$

be its lifting to $\widetilde{\Omega}_{0}(M) \times \widetilde{\Omega}_{0}(M)$ under the projection map

$$
\widetilde{\Omega}_{0}(M) \times \widetilde{\Omega}_{0}(M) \rightarrow \Omega_{0}(M) \times \Omega_{0}(M) .
$$

We consider the spaces

$$
\widetilde{\mathcal{L}}_{2}(M):=\left(\widetilde{\Omega}_{0}(M) \times \widetilde{\Omega}_{0}(M)\right) \backslash \widetilde{\Delta}
$$

and

$$
\widetilde{\mathcal{L}}_{2}(M) \times \mathcal{P}\left(\mathcal{H}_{m}(M) ; H_{1}, H_{2}\right) \times[0,1]=: \widetilde{\Upsilon} .
$$

There is a natural product action by $\Gamma \times \Gamma$ on the product $\widetilde{\Omega}_{0}(M) \times \widetilde{\Omega}_{0}(M)$, which preserves the subset $\widetilde{\mathcal{L}}_{2}(M)$.

We define the map

$$
\widetilde{\Psi}: \widetilde{\Upsilon} \rightarrow T\left(\widetilde{\mathcal{L}}_{2}(M)\right) \times[0,1] \times \mathbb{R}
$$

by

$$
\begin{aligned}
\widetilde{\Psi}\left(\left[z_{1}, w_{1}\right],\left[z_{2}, w_{2}\right], \mathcal{H}, \eta\right)=( & \dot{z}_{1}-X_{H(\eta)}\left(z_{1}\right), \dot{z}_{2}-X_{H(\eta)}\left(z_{2}\right), \eta \\
& \left.\mathcal{A}_{H(\eta)}\left(\left[z_{1}, w_{1}\right]\right)-\mathcal{A}_{H(\eta)}\left(\left[z_{2}, w_{2}\right]\right)\right) .
\end{aligned}
$$

Note that there is a canonical identification

$$
T\left(\widetilde{\mathcal{L}}_{2}(M)\right) \times[0,1] \times \mathbb{R} \cong T\left(\mathcal{L}_{2}(M)\right) \times[0,1] \times \mathbb{R}
$$

as before and so we assume the image of $\widetilde{\Psi}$ lies $T\left(\mathcal{L}_{2}(M)\right) \times[0,1] \times \mathbb{R}$. The image of the map $\widetilde{\Psi}$ is invariant under the diagonal action $\Gamma$ on $\widetilde{\Upsilon}$ and so pushes down to the map

$$
\Psi: \Upsilon \rightarrow T\left(\mathcal{L}_{2}(M)\right) \times[0,1] \times \mathbb{R}
$$

where $\Upsilon:=\widetilde{\Upsilon} / \Gamma^{\text {diag }}$. In general $\Upsilon$ is not Hausdorff. Note that the quotient group

$$
\Gamma^{q u o t}:=(\Gamma \times \Gamma) / \Gamma^{\text {diag }}
$$

naturally acts on the domain and on the range of the map $\Psi$, with respect to which $\Psi$ is equivariant: the action of $\left(A_{1}, A_{2}\right) \in \Gamma \times \Gamma$ on $T\left(\mathcal{L}_{2}(M)\right) \times[0,1] \times \mathbb{R}$ is given by

$$
(\xi, \eta, a) \mapsto\left(\xi, \eta, a-\omega\left(A_{1} \# \bar{A}_{2}\right)\right)
$$


It follows, again by a standard calculation of the linearization of $\widetilde{\Psi}, \Psi$ is transverse to

$$
o_{T \mathcal{L}_{2}(M)} \times[0,1] \times\{0\} \subset T \mathcal{L}_{2}(M) \times[0,1] \times \mathbb{R} .
$$

In particular, we know that

$$
\mathcal{W}:=\Psi^{-1}\left(o_{T \mathcal{L}_{2}(M)} \times[0,1] \times\{0\}\right)
$$

is a smooth submanifold of $\Upsilon$.

Now we consider the projection

$$
\Pi: \mathcal{W} \subset \Upsilon \rightarrow \mathcal{P}\left(\mathcal{H}_{m}(M) ; H_{1}, H_{2}\right) .
$$

One can check that this is a Fredholm map of index 0. By the Sard-Smale theorem, there is a dense subset of $\mathcal{H}$ 's such that as long as it is non-empty,

$$
\Pi^{-1}(\mathcal{H})=: W(\mathcal{H})
$$

is a smooth zero dimensional manifold.

Furthermore by the dimension counting argument, one proves that the phenomena of multiple (with multiplicity more than 2) crossing or of having a nonempty intersection $\operatorname{Sing}(\mathcal{H}) \cap \mathcal{C r o s s}^{n d}(\mathcal{H}) \neq \emptyset$ generically have negative codimension and so can be avoided over a residual subset of $\mathcal{P}\left(\mathcal{H}_{m}(M) ; H_{1}, H_{2}\right)$, which we denote by $\mathcal{P}^{g c}\left(\mathcal{H}_{m}(M) ; H_{1}, H_{2}\right)$.

Now consider the map

$$
p: W(\mathcal{H}) \subset \widetilde{\mathcal{L}}_{2}(M) \times[0,1] \rightarrow[0,1] \times \mathbb{R}
$$

defined by

$$
\left(\left[z_{1}, w_{1}\right],\left[z_{2}, w_{2}\right], \eta\right) \rightarrow\left(\eta, \mathcal{A}_{H(\eta)}\left(\left[z_{1}, w_{1}\right]\right)\right)
$$

for which the diagram

$$
\begin{array}{ccc}
W(\mathcal{H}) & \longrightarrow & {[0,1] \times \mathbb{R}} \\
\downarrow & & \downarrow \\
{[0,1]} & \equiv & {[0,1]}
\end{array}
$$

commutes, and is equivariant under the action of $\Gamma^{\text {diag }}$ on $W(\mathcal{H})$, and the action (3.15). The image of $p$ is precisely the set of crossings in the Cerf diagram $\Sigma(\mathcal{H})$. Now the theorem follows by making a choice of any homotopy $\mathcal{H}$ from $\mathcal{P}^{\operatorname{Cerf}}\left(\mathcal{H}_{m}(M) ; H_{1}, H_{2}\right)$.

For the completeness's sake, we would like to consider the slopes of the branches at a crossing. Note that nondegenerate crossing implies that their slopes are different.

Suppose that $\left[z_{\eta}, w_{\eta}\right]$ locally parameterizes a branch at $\eta=\eta_{0}$. The slope of the branch at $\eta$ is given by

$$
\frac{d}{d \eta}\left(\mathcal{A}_{H(\eta)}\left(\left[z_{\eta}, w_{\eta}\right]\right)\right) .
$$


Using the fact that $z_{\eta}$ is a Hamiltonian periodic orbit of $H(\eta)$, we compute

$$
\begin{aligned}
\frac{d}{d \eta}\left(\mathcal{A}_{H(\eta)}\left(\left[z_{\eta}, w_{\eta}\right]\right)\right) & =d \mathcal{A}_{H(\eta)}\left(\left[z_{\eta}, w_{\eta}\right]\right)-\int_{0}^{1} \frac{\partial H}{\partial \eta}\left(t, z_{\eta}(t)\right) d t \\
& =-\int_{0}^{1} \frac{\partial H}{\partial \eta}\left(t, z_{\eta}(t)\right) d t .
\end{aligned}
$$

We remark that the slopes of the branches of periodic orbits are the same as those of their liftings to $\widetilde{\Omega}_{0}(M)$.

Definition 3.8. Suppose that $H_{\alpha}, H_{\beta}$ are two nondegenerate Hamiltonians. We call a homotopy $\mathcal{H}$ between them a Cerf homotopy or a Cerf family if $\mathcal{H}$ satisfies the properties described in Theorem 3.7 and in Proposition 3.8. We call a point

$$
\eta \in[0,1] \backslash \operatorname{Sing}(\mathcal{H})
$$

Cerf regular.

By definition, we have

$$
\mathcal{P}^{\operatorname{Cerf}}\left(\mathcal{H}_{m}(M) ; H_{1}, H_{2}\right):=\mathcal{P}^{r e g}\left(\mathcal{H}_{m}(M) ; H_{1}, H_{2}\right) \cap \mathcal{P}^{g c}\left(\mathcal{H}_{m}(M) ; H_{1}, H_{2}\right) .
$$

From now on, we will always assume that $\mathcal{H}$ is a Cerf homotopy, unless otherwise stated.

For the later purpose, we introduce a concept of sub-homotopy, which will play a crucial role in our proof of the spectrality.

Definition 3.9. For each given pair $\eta_{1}<\eta_{2} \in[0,1]$, we consider the homotopy $\mathcal{H}_{\eta_{1} \eta_{2}}$ of $\mathcal{H}$ between $H\left(\eta_{1}\right)$ and $H\left(\eta_{2}\right)$ defined by the reparameterization

$$
\mathcal{H}_{\eta_{1} \eta_{2}}: s \mapsto H^{s}=H\left((1-s) \eta_{1}+s \eta_{2}\right) .
$$

We call any such a homotopy $\mathcal{H}_{\eta_{1} \eta_{2}}$ a sub-homotopy of $\mathcal{H}$. When $\eta_{1}>\eta_{2}$, we define the corresponding sub-homotopy by

$$
\mathcal{H}_{\eta_{1} \eta_{2}}:=\left(\mathcal{H}^{-1}\right)_{\eta_{2} \eta_{1}}
$$

where $\mathcal{H}^{-1}$ is the time reversal homotopy defined by

$$
\mathcal{H}^{-1}: \eta \mapsto H(1-\eta) .
$$

\section{§4. Sub-homotopies and transversality}

\subsection{Definition of the Floer boundary map, re-visited}

Suppose $H$ is a nondegenerate one-periodic Hamiltonian function and $J$ be a one-periodic one-parameter family of compatible almost complex structure. We denote by

$$
j_{\omega}=C^{\infty}\left([0,1], \mathcal{J}_{\omega}\right)
$$

the set of such families of almost complex structures. We first recall the construction of the Floer boundary map and the transversality conditions needed to define the Floer homology $H F_{*}(H, J)$ of the pair. We will also add some 
novel elements in the exposition of the construction, which are needed for our formulation of the transversality problem of sub-homotopies.

The following definition is useful for the later discussion.

Definition 4.1. Let $z, z^{\prime} \in \operatorname{Per}(H)$. We denote by $\pi_{2}\left(z, z^{\prime}\right)$ the set of homotopy classes of smooth maps

$$
u:[0,1] \times S^{1}:=T \rightarrow M
$$

relative to the boundary

$$
u(0, t)=z(t), \quad u(1, t)=z^{\prime}(t) .
$$

We denote by $[u] \in \pi_{2}\left(z, z^{\prime}\right)$ its homotopy class and by $C$ a general element in $\pi_{2}\left(z, z^{\prime}\right)$.

We define by $\pi_{2}(z)$ to be the set of relative homotopy classes of the maps

$$
w: D^{2} \rightarrow M ;\left.\quad w\right|_{\partial D^{2}}=z .
$$

We note that there is a natural action of $\pi_{2}(M)$ on $\pi_{2}(z)$ and $\pi_{2}\left(z, z^{\prime}\right)$ by the obvious operation of a 'gluing a sphere'. Furthermore there is a natural map of $C \in \pi_{2}\left(z, z^{\prime}\right)$

$$
(\cdot) \# C: \pi_{2}(z) \rightarrow \pi_{2}\left(z^{\prime}\right)
$$

induced by the gluing map

$$
w \mapsto w \# u .
$$

More specifically we will define the map $w \# u: D^{2} \rightarrow M$ in the polar coordinates $(r, \theta)$ of $D^{2}$ by the formula

$$
w \# u:(r, \theta)= \begin{cases}w(2 r, \theta) & \text { for } 0 \leq r \leq \frac{1}{2} \\ w(2 r-1, \theta) & \text { for } \frac{1}{2} \leq r \leq 1\end{cases}
$$

once and for all. There is also the natural gluing map

$$
\begin{gathered}
\pi_{2}\left(z_{0}, z_{1}\right) \times \pi_{2}\left(z_{1}, z_{2}\right) \rightarrow \pi_{2}\left(z_{0}, z_{2}\right) \\
\left(u_{1}, u_{2}\right) \mapsto u_{1} \# u_{2} .
\end{gathered}
$$

We also explicitly represent the map $u_{1} \# u_{2}: T \rightarrow M$ in the standard way once and for all similarly to (4.1).

Definition 4.2. We define the relative Conley-Zehnder index of $C \in \pi_{2}\left(z, z^{\prime}\right)$ by

$$
\mu_{H}\left(z, z^{\prime} ; C\right)=\mu_{H}([z, w])-\mu_{H}\left(\left[z^{\prime}, w \# C\right]\right)
$$

for a (and so any) representative $u:[0,1] \times S^{1} \times M$ of the class $C$. We will also write $\mu_{H}(C)$, when there is no danger of confusion on the boundary condition.

It is easy to see that the right hand side of (4.2) does not depend on the choice of bounding disc $w$ of $z$, and so the function

$$
\mu_{H}: \pi_{2}\left(z, z^{\prime}\right) \rightarrow \mathbb{Z}
$$


is well-defined.

Remark 4.3. In fact, the function $\mu_{H}: \pi_{2}\left(z, z^{\prime}\right) \rightarrow \mathbb{Z}$ can be defined without assuming $z_{0}, z_{1}$ being contractible, as long as $z_{0}$ and $z_{1}$ lie in the same component of $\Omega(M)$ : For any given map $u: T \rightarrow M$, choose a marked symplectic trivialization

$$
\Phi: u^{*} T M \rightarrow T \times \mathbb{R}^{2 n}
$$

that satisfies

$$
\left.\Phi \circ \Phi^{-1}\right|_{[0,1] \times\{1\}}=i d .
$$

We know that $z_{0}(t)=\phi_{H}^{t}\left(p_{0}\right)$ and $z_{1}(t)=\phi_{H}^{t}\left(p_{1}\right)$ for $p_{0}, p_{1} \in \operatorname{Fix}\left(\phi_{H}^{1}\right)$. Then we have two maps

$$
\alpha_{\Phi, i}:[0,1] \rightarrow S p(2 n), \quad i=0,1
$$

such that

$$
\Phi \circ d \phi_{H}^{t}\left(p_{i}\right) \circ \Phi^{-1}(i, t, v)=\left(i, t, \alpha_{\Phi, i}(t) v\right)
$$

for $v \in \mathbb{R}^{2 n}$ and $t \in[0,1]$. By the nondegeneracy of $H$, the maps $\alpha_{\Phi, i}$ define elements in $\mathcal{S} P^{*}(1)$. (See the Appendix A.1 for the definition of $\mathcal{S} P^{*}(1)$.) Then we define

$$
\mu_{H}\left(z, z^{\prime} ; C\right):=\mu_{C Z}\left(\alpha_{\Phi, 0}\right)-\mu_{C Z}\left(\alpha_{\Phi, 1}\right) .
$$

It is easy to check that this definition does not depend on the choice of marked symplectic trivializations.

We now denote by

$$
\mathcal{M}\left(H, J ; z, z^{\prime} ; C\right)
$$

the set finite energy solutions of

$$
\frac{\partial u}{\partial \tau}+J\left(\frac{\partial u}{\partial t}-X_{H}(u)\right)=0
$$

with the asymptotic condition and the homotopy condition

$$
u(-\infty)=z, \quad u(\infty)=z^{\prime} ; \quad[u]=C .
$$

Here we remark that although $u$ is a priori defined on $\mathbb{R} \times S^{1}$, it can be compactified into a continuous map $\bar{u}:[0,1] \times S^{1} \rightarrow M$ with the corresponding boundary condition due to the exponential decay property of solutions $u$ of (4.3), recalling we assume $H$ is nondegenerate. We will call $\bar{u}$ the compactified map of $u$. By some abuse of notation, we will also denote by $[u]$ the class $[\bar{u}] \in \pi_{2}\left(z, z^{\prime}\right)$ of the compactified map $\bar{u}$.

We now recall that the Floer boundary map

$$
\partial_{(H, J)} ; C F_{k+1}(H) \rightarrow C F_{k}(H)
$$

is defined under the following conditions. (See [8], [10].)

Definition 4.4 (The boundary map). Let $H$ be nondegenerate. Suppose that $J$ satisfies the following conditions: 
(1) For any pair $\left(z_{0}, z_{1}\right) \subset \operatorname{Per}(H)$ satisfying

$$
\mu_{H}\left(z_{0}, z_{1} ; C\right)=\mu_{H}\left(\left[z_{0}, w_{0}\right]\right)-\mu_{H}\left(\left[z_{1}, w_{0} \# C\right]\right)=0,
$$

$\mathcal{M}\left(H, J ; z_{0}, z_{1} ; C\right)=\emptyset$ unless $z_{0}=z_{1}$ and $C=0$. When $z_{0}=z_{1}$ and $C=0$, the only solutions are the stationary solution, i.e., $u(\tau) \equiv z_{0}=$ $z_{1}$ for all $\tau \in \mathbb{R}$.

(2) For any pair $\left(z_{0}, z_{1}\right) \subset \operatorname{Per}(H)$ and a homotopy class $C \in \pi_{2}\left(z_{0}, z_{1}\right)$ satisfying

$$
\mu_{H}\left(z_{0}, z_{1} ; C\right)=1,
$$

$\mathcal{M}\left(H, J ; z_{0}, z_{1} ; C\right) / \mathbb{R}$ is transverse and compact and so a finite set. We denote

$$
n\left(H, J ; z_{0}, z_{1} ; C\right)=\#\left(\mathcal{M}\left(H, J ; z_{0}, z_{1} ; C\right) / \mathbb{R}\right)
$$

the algebraic count of the elements of the space $\mathcal{M}\left(H, J ; z_{0}, z_{1} ; C\right) / \mathbb{R}$. We set $n\left(H, J ; z_{0}, z_{1}: C\right)=0$ otherwise.

(3) For any pair $\left(z_{0}, z_{2}\right) \subset \operatorname{Per}(H)$ and $C \in \pi_{2}\left(z_{0}, z_{2}\right)$ satisfying

$$
\mu_{H}\left(z_{0}, z_{2} ; C\right)=2,
$$

$\mathcal{M}\left(H, J ; z_{0}, z_{2} ; C\right) / \mathbb{R}$ can be compactified into a smooth one-manifold with boundary comprising the collection of the broken trajectories

$$
\left[u_{1}\right] \#_{\infty}\left[u_{2}\right],
$$

where $u_{1} \in \mathcal{M}\left(H, J ; z_{0}, y: C_{1}\right)$ and $u_{2} \in \mathcal{M}\left(H, J ; y, z_{2}: C^{2}\right)$ for all possible $y \in \operatorname{Per}(H)$ and $C_{1} \in \pi_{2}\left(z_{0}, y\right), C_{2} \in \pi_{2}\left(y, z_{2}\right)$ satisfying

$$
C_{1} \# C_{2}=C ; \quad\left[u_{1}\right] \in \mathcal{M}\left(H, J ; z_{0}, y ; C_{1}\right) / \mathbb{R}, \quad\left[u_{2}\right] \in \mathcal{M}\left(H, J ; y, z_{2} ; C_{2}\right) / \mathbb{R}
$$

and

$$
\mu_{H}\left(z_{0}, y ; C_{1}\right)=\mu_{H}\left(y, z_{2} ; C_{2}\right)=1 .
$$

Here we denote by $[u]$ the equivalence class represented by $u$.

We call any such $J H$-regular and call any such pair $(H, J)$ Floer regular.

The upshot is that for a Floer regular pair $(H, J)$ the Floer boundary map

$$
\partial=\partial_{(H, J)}: C F_{*}(H) \rightarrow C F_{*}(H)
$$

is defined and satisfies $\partial \partial=0$ and so the Floer homology $H F(H, J):=$ ker $\partial /$ im $\partial$ is defined. For any given nondegenerate $H$, the set of $H$-regular $J$ 's is dense in $j_{\omega}$ under the assumption of semi-positivity. (See [8], [10] for the proof.) We denote by

$$
j_{\omega}^{r e g}(H) \subset j_{\omega}=C^{\infty}\left([0,1], \mathcal{J}_{\omega}\right)
$$

the set of $H$-regular $J$ 's. 


\subsection{Definition of the Floer chain map, re-visited}

Now we study the Floer regularity of the triple $(\mathcal{H}, j ; \rho)$. We need to study an intermediate problem. Consider the pair $\left(\mathcal{H}_{\mathbb{R}}, j_{\mathbb{R}}\right)$ of maps

$$
\begin{aligned}
\mathcal{H}_{\mathbb{R}}: \mathbb{R} \rightarrow C_{m}^{\infty}([0,1] \times M):=\mathcal{H}_{m}(M) \\
j_{\mathbb{R}}: \mathbb{R} \rightarrow j_{\omega}
\end{aligned}
$$

that are asymptotically constant, i.e., such that there exists $R>0$ such that

$$
H(\tau) \equiv H( \pm \infty), \quad j(\tau) \equiv j( \pm \infty)
$$

for $\tau$ with $|\tau| \geq R$. To each such a pair, we associate non-autonomous analog to (4.3) still with the condition (4.4).

Definition 4.5 (The chain map). We say that $\left(\mathcal{H}_{\mathbb{R}}, j_{\mathbb{R}}\right)$ is Floer regular if the following holds:

(1) For any pair $z_{0} \in \operatorname{Per}\left(H_{0}\right)$ and $z_{1} \subset \operatorname{Per}\left(H_{1}\right)$ satisfying

$$
\mu_{\mathcal{H}_{\mathbb{R}}}\left(z_{0}, z_{1} ; C\right)=0,
$$

$\mathcal{M}\left(\mathcal{H}_{\mathbb{R}}, j_{\mathbb{R}} ; z_{0}, z_{1} ; C\right)$ is transverse and compact, and so a finite set. We denote

$$
n\left(\mathcal{H}_{\mathbb{R}}, j_{\mathbb{R}} ; z_{0}, z_{1} ; C\right)=\#\left(\mathcal{M}\left(\mathcal{H}_{\mathbb{R}}, j_{\mathbb{R}} ; z_{0}, z_{1} ; C\right)\right)
$$

the algebraic count of the elements in $\mathcal{M}\left(\mathcal{H}_{\mathbb{R}}, j_{\mathbb{R}} ; z_{0}, z_{1} ; C\right)$. We set $n\left(\mathcal{H}_{\mathbb{R}}, j_{\mathbb{R}} ; z_{0}, z_{1}: C\right)=0$ otherwise.

(2) For any pair $z_{0} \in \operatorname{Per}\left(H_{0}\right)$ and $z_{1} \in \operatorname{Per}\left(H_{1}\right)$ satisfying

$$
\mu_{\mathcal{H}_{\mathbb{R}}}\left(z_{0}, z_{2} ; C\right)=1,
$$

$\mathcal{M}\left(H, J ; z_{0}, z_{2} ; C\right)$ is transverse and can be compactified into a smooth one-manifold with boundary comprising the collection of the broken trajectories

where

$$
u_{1} \#{ }_{\infty} u_{2},
$$

$$
\begin{gathered}
\left(u_{1}, u_{2}\right) \in \mathcal{M}\left(\mathcal{H}_{\mathbb{R}}, j_{\mathbb{R}} ; z_{0}, y: C_{1}\right) \times \mathcal{M}\left(H(\infty), J(\infty) ; y, z_{2}: C^{2}\right) \\
\mu_{\mathcal{H}_{\mathbb{R}}}\left(z_{0}, y ; C_{1}\right)=0, \mu_{H}\left(y, z_{2} ; C_{2}\right)=1
\end{gathered}
$$

or

$$
\begin{gathered}
\left(u_{1}, u_{2}\right) \in \mathcal{M}\left(H(-\infty), J(-\infty) ; z_{0}, y: C^{1}\right) \times \mathcal{M}\left(\mathcal{H}_{\mathbb{R}}, j_{\mathbb{R}} ; y, z_{2}: C_{1}\right) \\
\mu_{\mathcal{H}_{\mathbb{R}}}\left(z_{0}, y ; C_{1}\right)=1, \mu_{H}\left(y, z_{2} ; C_{2}\right)=0
\end{gathered}
$$

and $C_{1} \# C_{2}=C$ for all possible such $y \in \operatorname{Per}(H)$ and $C_{1} \in \pi_{2}\left(z_{0}, y\right)$, $C_{2} \in \pi_{2}\left(y, z_{2}\right)$.

We say that $\left(\mathcal{H}_{\mathbb{R}}, j_{\mathbb{R}}\right)$ are Floer regular if it satisfies these conditions.

Again for any fixed $\mathcal{H}_{\mathbb{R}}$, the residual property of $j_{\mathbb{R}}$ 's for which $\left(\mathcal{H}_{\mathbb{R}}, j_{\mathbb{R}}\right)$ are regular is well-known for the semi-positive case. (See [8], [10].) 
Now suppose that $\mathcal{H}$ is a homotopy connecting two nondegenerate Hamiltonians $H_{0}$ and $H_{1}$. We denote

$$
\mathcal{P}\left(j_{\omega}\right):=C^{\infty}\left([0,1], j_{\omega}\right)
$$

the set of smooth one-parameter family $j=\{J(s)\}_{0 \leq s \leq 1}$ with $J(s) \in j_{\omega}$. We define a function $\rho: \mathbb{R} \rightarrow[0,1]$ of the type

$$
\rho(\tau)= \begin{cases}0 & \text { for } \tau \leq-R \\ 1 & \text { for } \tau \geq R\end{cases}
$$

for some $R>0$. We call $\rho$ a monotone cut-off function if it satisfies $\rho^{\prime}(\tau) \geq 0$ for all $\tau$ 's in addition.

Each such pair $(\mathcal{H}, j)$ and a cut-off function $\rho$ define a pair

$$
\mathcal{H}_{\mathbb{R}}=\mathcal{H}^{\rho}, \quad j_{\mathbb{R}}=j^{\rho},
$$

where $\mathcal{H}^{\rho}$ is the reparameterized homotopy $\mathcal{H}^{\rho}=\left\{H^{\rho}\right\}_{\tau \in \mathbb{R}}$ defined by

$$
\tau \mapsto H^{\rho}(\tau, t, x)=H(\rho(\tau), t, x) .
$$

We call $\mathcal{H}^{\rho}$ the $\rho$-elongation of $\mathcal{H}$ or the $\rho$-elongated homotopy of $\mathcal{H}$. The same definition applies to $j$. Therefore such a triple $(\mathcal{H}, j ; \rho)$ associates the non-autonomous equation

$$
\left\{\begin{array}{l}
\frac{\partial u}{\partial \tau}+J^{\rho(\tau)}\left(\frac{\partial u}{\partial t}-X_{H^{\rho(\tau)}}(u)\right)=0 \\
\lim _{\tau \rightarrow-\infty} u(\tau)=z^{-}, \lim _{\tau \rightarrow \infty} u(\tau)=z^{+}
\end{array}\right.
$$

with the boundary condition

$$
u(-\infty)=z_{0}, \quad u(\infty)=z_{1} .
$$

We denote by

$$
\mathcal{M}\left((\mathcal{H}, j ; \rho) ; z_{0}, z_{1} ; C\right)
$$

the set of finite energy solutions of (4.5)-(4.6) satisfying the topological condition $[u]=C$ in $\pi_{2}\left(z_{0}, z_{1}\right)$. We say that $(\mathcal{H}, j ; \rho)$ is Floer regular if the $\rho$ elongation $\left(\mathcal{H}^{\rho}, j^{\rho}\right)$ is Floer regular in the sense of Definition 4.5.

\subsection{Transversality of sub-homotopies}

With these preparations, we now launch our main study of the transversality question on the sub-homotopies of a given pair $(\mathcal{H}, j)$.

Let $\mathcal{H}$ be a Cerf homotopy and $j \in \mathcal{P}\left(j_{\omega}\right)$ be given. As for the homotopy $\mathcal{H}$ of Hamiltonian functions, we define the sub-homotopy $j_{\eta \eta^{\prime}}$ by

$$
s \in[0,1] \mapsto J\left((1-s) \eta+s \eta^{\prime}\right)
$$

for each given $0 \leq \eta \leq \eta^{\prime} \leq 1$. When $\eta>\eta^{\prime}$, we define

$$
j_{\eta \eta^{\prime}}:=\left(j^{-1}\right)_{\eta^{\prime} \eta} \text {. }
$$


The main purpose of the present section is to study the question whether one can define the Floer chain map

$$
h_{\left(\eta \eta^{\prime} ; \rho\right)}: C F_{*}(H(\eta)) \rightarrow C F_{*}\left(H\left(\eta^{\prime}\right)\right)
$$

for sufficiently many points of $\eta^{\prime} \in[0,1]$, when there are given a generic homotopy $(\mathcal{H}, j)$ and a Cerf regular point $\eta \in[0,1]$.

The following theorem is the main theorem in that regard.

Theorem 4.6. Let $\mathcal{H} \in \mathcal{P}^{\operatorname{Cerf}}\left(\mathcal{H}_{m}(M) ; H_{\alpha}, H_{\beta}\right)$ be a Cerf homotopy connecting two nondegenerate Hamiltonians $H_{\alpha}, H_{\beta}$. Let $J_{i}, i=\alpha, \beta\left(H_{i}, J_{i}\right)$ be Floer regular and fix a cut-off function $\rho: \mathbb{R} \rightarrow[0,1]$. Then we have the following:

(1) There exists a dense subset

$$
\mathcal{P}^{\operatorname{tran}}\left(j_{\omega} ; \mathcal{H}\right) \subset \mathcal{P}\left(j_{\omega}\right)
$$

such that for any element $j$ from $\mathcal{P}^{\text {tran }}\left(j_{\omega} ; \mathcal{H}\right)$ there exists a residual subset of $[0,1]$ containing $\{0,1\}$, denoted by

$$
I(\mathcal{H}, j) \subset[0,1],
$$

at each point $\eta$ of which the pair $(H(\eta), J(\eta))$ is Floer regular.

(2) For any $\eta \in I(\mathcal{H}, j)$, there exists a residual subset

$$
\mathcal{P}^{s u b}\left(j_{\omega}, \mathcal{H} ; \eta\right) \subset \mathcal{P}^{\text {tran }}\left(j_{\omega} ; \mathcal{H}\right)
$$

such that for any $j \in \mathcal{P}^{s u b}\left(j_{\omega} ; \mathcal{H} ; \eta\right)$ there exists a residual subset

$$
I(\mathcal{H}, j ; \eta) \subset I(\mathcal{H}, j) \subset[0,1]
$$

such that for any $\eta^{\prime} \in I(\mathcal{H}, j ; \eta)$ the triple

$$
\left(\mathcal{H}_{\eta \eta^{\prime}}, j_{\eta \eta^{\prime}} ; \rho\right)
$$

is Floer regular and hence the Floer chain map

$$
h_{\left(\mathcal{H}_{\eta \eta^{\prime}}, j_{\eta \eta^{\prime}} ; \rho\right)}:=h_{\left(\mathcal{H}_{\eta \eta^{\prime}}^{\rho}, j_{\eta \eta^{\prime}}^{\rho}\right)}: C F_{*}\left(H_{0}\right) \rightarrow C F_{*}\left(H_{1}\right)
$$

is defined and satisfies

$$
h_{\left(\mathcal{H}_{\eta \eta^{\prime}}, j_{\eta \eta^{\prime}} ; \rho\right)} \circ \partial_{\left(H_{\alpha}, J_{\alpha}\right)}=\partial_{\left(H_{\beta}, J_{\beta}\right)} \circ h_{\left(\mathcal{H}_{\eta \eta^{\prime}}, j_{\eta \eta^{\prime}} ; \rho\right)} .
$$

The rest of the section will be occupied by the proof of this theorem. We fix a Cerf homotopy $\mathcal{H}$ and a cut-off function $\rho$.

We consider the case (1) first. For each fixed $z_{0} \in \operatorname{Per}\left(H_{0}\right)$ and $z_{1} \in \operatorname{Per}\left(H_{1}\right)$, and a class $C \in \pi_{2}\left(z_{0}, z_{1}\right)$, we consider the space

$$
C^{\infty}\left(z_{0}, z_{1} ; C\right):=\left\{u: \mathbb{R} \times S^{1} \rightarrow M \mid u \text { satisfies }(4.6) \text { and } E_{(H, J)}(u)<\infty\right\}
$$

and its $W^{1, p}$-completion with respect to a suitably weighted Sobolev norm on $C^{\infty}\left(z_{0}, z_{1} ; C\right)$. (See [5].) We denote the corresponding weighted $W^{1, p}$-space as

$$
W^{1, p}\left(z_{0}, z_{1} ; C\right) \text {. }
$$


Then we consider the assignment

$$
(u, J) \mapsto \frac{\partial u}{\partial \tau}+J^{\rho(\tau)}\left(\frac{\partial u}{\partial t}-X_{H^{\rho(\tau)}}(u)\right)
$$

as a section of a vector bundle $\mathcal{E}\left(z_{0}, z_{1} ; C\right)$ over $W^{1, p}\left(z_{0}, z_{1} ; C\right) \times j_{\omega}$ whose fiber is given by

$$
L^{p}\left(z_{0}, z_{1} ; C\right):=L^{p}\left(\Lambda^{0,1}\left(u^{*} T M\right)\right) .
$$

We denote this section by

$$
\bar{\partial}_{H}: W^{1, p}\left(z_{0}, z_{1} ; C\right) \times j_{\omega} \rightarrow \mathcal{E}\left(z_{0}, z_{1} ; C\right),
$$

and denote by $\underline{0}$ the zero section of the vector bundle $\mathcal{E}\left(z_{0}, z_{1} ; C\right)$. Then it is well-known [5], [8] that the covariant linearization of $\bar{\partial}_{H}$ is surjective and so the zero set

$$
\mathcal{M}\left(H ; z_{0}, z_{1} ; C\right):=\left(\bar{\partial}_{H}\right)^{-1}(\underline{0}) \subset W^{1, p}\left(z_{0}, z_{1} ; C\right) \times j_{\omega}
$$

is a smooth submanifold whose image is indeed contained in $C^{\infty}\left(z_{0}, z_{1} ; C\right) \times j_{\omega}$. Furthermore the projection

$$
\Pi_{\left(H ; z_{0}, z_{1} ; C\right)}: \mathcal{M}\left(H ; z_{0}, z_{1} ; C\right) \rightarrow j_{\omega}
$$

is a Fredholm map of index $\mu_{H}(C)$. By the Sard-Smale theorem, the set $j_{\omega}^{r e g}(H) \subset j_{\omega}$ is a residual subset and so dense.

To prove $(1)$, we consider $j:[0,1] \rightarrow j_{\omega}$ with $j(0)=J_{0}$ and $j(1)=J_{1}$. Then applying the Sard-Smale theorem, it is enough to consider the set of smooth paths $j:[0,1] \rightarrow j_{\omega}$ that are transverse to $\Pi_{\left(H ; z_{0}, z_{1} ; C\right)}$ for all triple $\left(z_{0}, z_{1} ; C\right)$. Since $H$ is assumed to be nondegenerate, there are only finitely many pairs $\left(z_{0}, z_{1}\right)$ and so only countably many possible triples $\left(z_{0}, z_{1} ; C\right)$. We denote by

$$
\mathcal{P}^{\operatorname{tran}}\left(j_{\omega} ; \mathcal{H}\right)
$$

the set of such $j=\{J(\eta)\}_{0 \leq \eta \leq 1}$. Then

$$
\mathcal{M}\left(\mathcal{H}, j ; z_{0}, z_{1} ; C\right):=\bigcup_{\eta \in[0,1]} \mathcal{M}\left(H(\eta), J(\eta) ; z_{0}, z_{1} ; C\right) \subset[0,1] \times W^{1, p}\left(z_{0}, z_{1} ; C\right)
$$

is a smooth manifold of dimension $\mu_{H}(C)+1$. There is a canonical projection

$$
\pi_{j}: \mathcal{M}\left(\mathcal{H}, j ; z_{0}, z_{1} ; C\right) \rightarrow[0,1] .
$$

By the classical Sard theorem, the set of regular values of $\pi_{j}$, denoted by $I(\mathcal{H}, j) \subset[0,1]$, is residual and so dense. This finishes the proof of (1).

For the proof of (2), we first note that there is a natural map

$$
\mathcal{S} u b_{\eta}: \mathcal{P}\left(j_{\omega}\right) \times[0,1] \rightarrow \mathcal{P}\left(j_{\omega}\right)
$$

defined by taking the sub-homotopy

$$
\mathcal{S} u b_{\eta}\left(j, \eta^{\prime}\right)=j_{\eta \eta^{\prime}} ; \quad j_{\eta \eta^{\prime}}(s):=j\left((1-s) \eta+s \eta^{\prime}\right) .
$$

We need to study Floer regularity of the triple

$$
\left(\mathcal{H}_{\eta \eta^{\prime}}, j_{\eta \eta^{\prime}} ; \rho\right)
$$


Fix an element $j \in \mathcal{P}^{\text {tran }}\left(j_{\omega} ; \mathcal{H}\right)$. Note that by Definition 4.5 , the triple $\left(\mathcal{H}_{\eta \eta}, j_{\eta \eta} ; \rho\right)$ is Floer regular if $j \in \mathcal{P}^{\text {trans }}\left(j_{\omega} ; \mathcal{H}\right)$ and if the corresponding moduli spaces satisfying

$$
\mathcal{M}\left(\left(\mathcal{H}_{\eta \eta}, j_{\eta \eta} ; \rho\right) ; z, z^{\prime} ; C\right)=\emptyset
$$

except when $z=z^{\prime}$ and $C=\underline{0}$. In particular, the are only a finite number of moduli spaces to consider, each of which consists of a single stationary element.

Since the structure of the set $\operatorname{Per}\left(H\left(\eta^{\prime}\right)\right)$ will vary as $\eta^{\prime}$ changes, we need to find a way of encoding the asymptotic conditions of (4.5) corresponding to $\left(\mathcal{H}_{\eta \eta^{\prime}}, j_{\eta \eta^{\prime}}\right)$ as $\eta^{\prime}$ varies. For example it may experience bifurcations as $\eta^{\prime}$ changes. Here enters again the property of Cerf homotopy $\mathcal{H}$ in a crucial way.

We partition $[0,1]$ into

$$
0=s_{0}<s_{1}<s_{2}<\cdots<s_{k_{1}}=1,
$$

where $\operatorname{Sing}(\mathcal{H})=\left\{s_{1}, \ldots, s_{k}\right\}$, and denote $I_{n}=\left(s_{n-1}, s_{n}\right)$. Since a bifurcation of periodic orbits occurs only at the points $s_{n}$, we can smoothly parameterize $\operatorname{Per}(H(\eta))$ on each $I_{n}$. For each given pair $\left(n, n^{\prime}\right)$ and a pair of points $\left(\eta, \eta^{\prime}\right) \in$ $I_{n} \times I_{n^{\prime}}$, we denote by $\left(z, z^{\prime}\right)$ the pair of branches $z=z(\eta)$ for $\eta \in I_{n}$ and $z^{\prime}=z^{\prime}\left(\eta^{\prime}\right)$ for $\eta \in I_{n^{\prime}}$. For each given such a pair, we denote by

$$
\mathcal{M}_{n}^{n^{\prime}}\left(\left(\mathcal{H}_{\eta \eta}, j_{\eta \eta} ; \rho\right) ; z, z^{\prime} ; C\right)
$$

the corresponding moduli space of solutions of (4.5). Therefore we need to consider only a finite number of possibilities. To study the transversality of (4.13), we need to set up the Fredholm theory for each given $\left(n, n^{\prime} ; z, z^{\prime}\right)$ and a given $C \in \pi_{2}\left(z, z^{\prime}\right)$.

Let $\eta \in[0,1]$ be given and $\eta \in I_{n}$, and fix another interval $I_{n^{\prime}}$. We consider the assignment

$$
\bar{\partial}_{(\mathcal{H} ; \eta)}:\left(u, j, \eta^{\prime}\right) \mapsto \bar{\partial}_{\left(\mathcal{H}_{\eta \eta^{\prime}}, j_{\eta \eta^{\prime}} ; \rho\right)}(u)
$$

for $\eta^{\prime} \in I_{n^{\prime}}$. The linearization is surjective as before and so the universal moduli space

$$
\left(\bar{\partial}_{(\mathcal{H} ; \eta)}\right)^{-1}(\underline{0})=: \mathcal{M}_{n}^{n^{\prime}}\left(\mathcal{H} ; \rho ; z, z^{\prime} ; C\right)
$$

is a smooth submanifold of

$$
C^{\infty}\left(\mathbb{R} \times S^{1} ; z, z^{\prime} ; C\right) \times \mathcal{P}\left(j_{\omega}\right) \times I_{n^{\prime}}
$$

The projection map

$$
\Pi_{\mathcal{H}}: \mathcal{M}_{n}^{n^{\prime}}\left(\mathcal{H} ; \rho ; z, z^{\prime} ; C\right) \rightarrow \mathcal{P}\left(j_{\omega}\right)
$$

is a Fredholm map of index $\mu_{\mathcal{H}_{\eta \eta^{\prime}}}(C)+1$ in general. We need to study the cases $\mu_{\mathcal{H} \eta \eta^{\prime}}(C)=0$ and $\mu_{\mathcal{H}_{\eta \eta^{\prime}}}(C)=1$. We will provide the details only for the case $\mu_{\mathcal{H}_{\eta \eta^{\prime}}}(C)=0$ and leave the other case to the readers.

Again by the Sard-Smale theorem, the regular values of $\Pi_{\mathcal{H}}$ is residual for each choice of $\left(n^{\prime}, z^{\prime} ; C\right)$ with $C \in \pi_{2}\left(z, z^{\prime}\right)$. We define by

$$
\mathcal{P}^{s u b}\left(j_{\omega} ; \mathcal{H} ; \eta\right)
$$


the intersection of the sets of regular values over all possible $\left(n^{\prime}, z^{\prime} ; C\right)$ and $\mathcal{P}^{\operatorname{tran}}\left(j_{\omega} ; \mathcal{H}\right)$. Certainly we have

$$
\mathcal{P}^{s u b}\left(j_{\omega} ; \mathcal{H} ; \eta\right) \subset \mathcal{P}^{\text {tran }}\left(j_{\omega} ; \mathcal{H}\right)
$$

and is a residual subset of $\mathcal{P}\left(j_{\omega}\right)$, since there are only countably many choices of $\left(n^{\prime}, z^{\prime} ; C\right)$.

By the definition of $\mathcal{P}^{s u b}\left(j_{\omega} ; \mathcal{H} ; \eta\right)$, the preimage

$$
\Pi_{\mathcal{H}}^{-1}(j)=\bigcup_{\eta^{\prime} \in I_{n^{\prime}}} \mathcal{M}_{n}^{n^{\prime}}\left(\left(\mathcal{H}_{\eta \eta^{\prime}}, j_{\eta \eta^{\prime}} ; \rho\right) ; z, z^{\prime} ; C\right) \subset C^{\infty}\left(z, z^{\prime} ; C\right) \times I_{n^{\prime}}
$$

is a smooth manifold of dimension 1 for each given $n^{\prime}$, which forms a fibration over $I_{n^{\prime}}$. We denote by

$$
\pi_{n}^{n^{\prime}}: \Pi_{\mathcal{H}}^{-1}(j) \rightarrow I_{n^{\prime}}
$$

the natural projection map. Denote by $I_{n^{\prime}}^{r e g}[n]$ the set of regular values of $\pi_{n}^{n^{\prime}}$. Then we have only to define $I(\mathcal{H}, j ; \eta)$ to the union

$$
I(\mathcal{H}, j ; \eta):=\bigcup_{n^{\prime}=1}^{k_{1}} I_{n^{\prime}}^{r e g}[n] .
$$

This proves the proof of (2) and so the theorem.

Similar discussion applies to the case $\mu_{\mathcal{H}_{\eta \eta^{\prime}}}(C)=1$ and omitted.

\section{$\S 5$. Composition law of Floer's chain maps, re-visited}

In this section, we will re-examine the well-known composition raw

$$
h_{\alpha \gamma}=h_{\beta \gamma} \circ h_{\alpha \beta}
$$

of the Floer's canonical isomorphism [8]

$$
h_{\alpha \beta}: H F_{*}\left(H_{\alpha}\right) \rightarrow H F_{*}\left(H_{\beta}\right) .
$$

We first carefully review this construction in the chain level. Although the isomorphism (5.2) in homology depends only on the end Hamiltonians $H_{\alpha}$ and $H_{\beta}$, the corresponding chain map depends on the homotopy $\mathcal{H}=\{H(\eta)\}_{0 \leq \eta \leq 1}$ between $H_{\alpha}$ and $H_{\beta}$, and also on the homotopy $j=\{J(\eta)\}_{0 \leq \eta \leq 1}$. Let us fix nondegenerate Hamiltonians $H_{\alpha}, H_{\beta}$ and a homotopy $\mathcal{H}$ between them. We also fix a homotopy $j=\{J(\eta)\}_{0 \leq \eta \leq 1}$ of compatible almost complex structures and a cut-off function $\rho: \mathbb{R} \rightarrow[0,1]$. The Floer chain map is defined by considering the non-autonomous equation

$$
\left\{\begin{array}{l}
\frac{\partial u}{\partial \tau}+J^{\rho(\tau)}\left(\frac{\partial u}{\partial t}-X_{H^{\rho(\tau)}}(u)\right)=0 \\
\lim _{\tau \rightarrow-\infty} u(\tau)=z^{-}, \lim _{\tau \rightarrow \infty} u(\tau)=z^{+}
\end{array}\right.
$$

with the condition that $w^{+}$and $w^{-} \# u$ are homotopic to each other relative to the boundary. We denote this condition by

$$
w^{+} \sim w^{-} \# u .
$$


One consequence of (5.4) is

$$
\left[z^{+}, w^{+}\right]=\left[z^{+}, w^{-} \# u\right] \quad \text { in } \quad \Gamma
$$

but the latter is a much weaker condition than the former. The asymptotic condition with (5.4) is equivalent to (4.6) corresponding to the class $C=[u]$.

Considering any such solution $u$ as a path in the covering space $\widetilde{\Omega}_{0}(M)$, we will also write the asymptotic condition as

$$
\lim _{\tau \rightarrow-\infty} u(\tau)=\left[z^{-}, w^{-}\right], \quad \lim _{\tau \rightarrow \infty} u(\tau)=\left[z^{+}, w^{+}\right] .
$$

$h_{(\mathcal{H}, j ; \rho)}$ has degree 0 and satisfies

$$
\partial_{\left(J^{1}, H^{1}\right)} \circ h_{(\mathcal{H}, j ; \rho)}=h_{(\mathcal{H}, j ; \rho)} \circ \partial_{\left(J^{0}, H^{0}\right)} .
$$

In general two such maps $h_{\left(\mathcal{H}, j ; \rho_{1}\right)}$ and $h_{\left(\mathcal{H}, j ; \rho_{2}\right)}$ are chain homotopic to each other in the sense of (5.7) below. In the end of this section, we will carefully study the dependance of this chain map on the cut-off functions.

Remark 5.1. One may directly consider the $\mathbb{R}$-family of Hamiltonians that are asymptotically constant as some literature do, which may remove the additional choice $\rho$ in the construction and be more natural in the point of view of Hamiltonian fibrations. However in this paper we prefer to use our approach because the usual homotopy is defined over $[0,1]$. Composing the homotopy with the cut-off function $\rho: \mathbb{R} \rightarrow[0,1]$ automatically makes the corresponding elongated homotopy asymptotically constant, which is a necessary requirement for the many parts of analysis of the perturbed Cauchy-Riemann equation: e.g., the gluing theorem and the index theorem of the solutions of the perturbed Cauchy-Riemann equation. In fact, using the cut-off function, we can consider the elongation of the sub-homotopy $\mathcal{H}_{\eta \eta^{\prime}}$ of a given homotopy $(\mathcal{H}, j)$ in one step by considering the functions $\rho$ of the type satisfying

$$
\begin{gathered}
\rho(\tau)= \begin{cases}\eta & \text { for } \tau \leq-R \\
\eta^{\prime} & \text { for } \tau \geq R\end{cases} \\
\eta \leq \rho(\tau) \leq \eta^{\prime} .
\end{gathered}
$$

We will elaborate this point elsewhere.

When we are given a homotopy $(\bar{j}, \overline{\mathcal{H}})$ of homotopies with $\bar{j}=\left\{j_{\kappa}\right\}, \overline{\mathcal{H}}=$ $\left\{\mathcal{H}_{\kappa}\right\}$, we also define the elongations $\mathcal{H}^{\bar{\rho}}$ of $\mathcal{H}_{\kappa}$ by a homotopy of cut-off functions $\bar{\rho}=\left\{\rho_{\kappa}\right\}$ : we have

$$
\mathcal{H}^{\bar{\rho}}=\left\{\mathcal{H}_{\kappa}^{\rho_{\kappa}}\right\}_{0 \leq \kappa \leq 1} .
$$

Consideration of the parameterized version of (5.3) for $0 \leq \kappa \leq 1$ defines the chain homotopy map

$$
H_{\overline{\mathcal{H}}}: C F_{*}\left(H_{\alpha}\right) \rightarrow C F_{*}\left(H_{\beta}\right)
$$

which has degree +1 and satisfies

$$
h_{\left(j_{1}, \mathcal{H}_{1} ; \rho_{1}\right)}-h_{\left(j_{0}, \mathcal{H}_{0}: \rho_{0}\right)}=\partial_{\left(J^{1}, H^{1}\right)} \circ H_{\overline{\mathcal{H}}}+H_{\overline{\mathcal{H}}} \circ \partial_{\left(J^{0}, H^{0}\right)} .
$$


Again the map $\mathcal{H}_{\overline{\mathcal{H}}}$ depends on the choice of a homotopy $\bar{j}$ and $\bar{\rho}=\left\{\rho_{\kappa}\right\}_{0 \leq \kappa \leq 1}$ connecting the two functions $\rho_{0}, \rho_{1}$. Therefore we will denote

$$
H_{\overline{\mathcal{H}}}=H_{(\overline{\mathcal{H}}, \bar{j} ; \bar{\rho})} .
$$

(5.7) in particular proves that two such chain maps (5.2) for different homotopies $\left(j_{0}, \mathcal{H}_{0} ; \rho_{0}\right)$ and $\left(j_{1}, \mathcal{H}_{1} ; \rho_{1}\right)$ connecting the same end points are chain homotopic [8] and so proves that the isomorphism (5.2) in homology is independent of the homotopies $(\overline{\mathcal{H}}, \bar{j})$ or of $\bar{\rho}$.

Now we re-examine the equation (5.3). The key analytic fact in the proof of (5.6) or (5.7) is an a priori upper bound of the energy

$$
E_{(\mathcal{H}, j ; \rho)}(u):=\frac{1}{2} \int_{-\infty}^{\infty} \int_{0}^{1}\left(\left|\frac{\partial u}{\partial \tau}\right|_{J^{\rho(\tau)}}^{2}+\left|\frac{\partial u}{\partial t}-X_{H^{\rho(\tau)}}(u)\right|_{J^{\rho(\tau)}}^{2}\right) d t d \tau
$$

for the solutions $u$ of (5.3) with (5.4). In this respect, we recall the following standard identity.

Lemma 5.2. Let $(\mathcal{H}, j)$ be any pair as above, not necessarily generic. Suppose that $u$ satisfies (5.3), has finite energy and satisfies

$$
\lim _{j \rightarrow \infty} u\left(\tau_{j}^{-}\right)=\left[z^{-}, w^{-}\right], \quad \lim _{j \rightarrow \infty} u\left(\tau_{j}^{+}\right)=\left[z^{+}, w^{+}\right]
$$

for some sequences $\tau_{j}^{ \pm}$with $\tau_{j}^{-} \rightarrow-\infty$ and $\tau_{j}^{+} \rightarrow \infty$. Then we have

$$
\begin{aligned}
& \mathcal{A}_{F}\left(\left[z^{+}, w^{+}\right]\right)-\mathcal{A}_{H}\left(\left[z^{-}, w^{-}\right]\right) \\
= & -\int\left|\frac{\partial u}{\partial \tau}\right|_{J^{\rho_{1}(\tau)}}^{2}-\int_{-\infty}^{\infty} \rho^{\prime}(\tau) \int_{0}^{1}\left(\left.\frac{\partial H^{s}}{\partial s}\right|_{s=\rho(\tau)}(t, u(\tau, t))\right) d t d \tau
\end{aligned}
$$

Corollary 5.3. Let $(\mathcal{H}, j)$ and $u$ be as in Lemma 5.2.

(1) Suppose that $\rho$ is monotone in addition. Then we have

$$
\begin{aligned}
\mathcal{A}_{F}\left(\left[z^{+}, w^{+}\right]\right)-\mathcal{A}_{H}\left(\left[z^{-}, w^{-}\right]\right) & \leq-\int\left|\frac{\partial u}{\partial \tau}\right|_{J^{\rho_{1}(\tau)}}^{2}+\int_{0}^{1}-\min _{x, s}\left(\frac{\partial H_{t}^{s}}{\partial s}\right) d t \\
& \leq \int_{0}^{1}-\min _{x, s}\left(\frac{\partial H_{t}^{s}}{\partial s}\right) d t .
\end{aligned}
$$

And (5.10) can be rewritten as the upper bound for the energy

$$
\begin{gathered}
\int\left|\frac{\partial u}{\partial \tau}\right|_{J^{\rho_{1}(\tau)}}^{2} \leq \mathcal{A}_{H}\left(\left[z^{+}, w^{+}\right]\right)-\mathcal{A}_{F}\left(\left[z^{-}, w^{-}\right]\right) \\
+\int_{0}^{1}-\min _{x, s}\left(\frac{\partial H_{t}^{s}}{\partial s}\right) d t .
\end{gathered}
$$

(2) For a general $\rho$, we instead have

$$
\mathcal{A}_{F}\left(\left[z^{+}, w^{+}\right]\right)-\mathcal{A}_{H}\left(\left[z^{-}, w^{-}\right]\right) \leq-\int\left|\frac{\partial u}{\partial \tau}\right|_{J^{\rho_{1}(\tau)}}^{2}+\int_{0}^{1} \max _{x, s}\left|\frac{\partial H_{t}^{s}}{\partial s}\right| d t
$$




$$
\leq \int_{0}^{1} \max _{x, s}\left|\frac{\partial H_{t}^{s}}{\partial s}\right| d t .
$$

And (5.13) can be rewritten as the upper bound for the energy

$$
\begin{aligned}
\int\left|\frac{\partial u}{\partial \tau}\right|_{J^{\rho_{1}(\tau)}}^{2} \leq & \mathcal{A}_{H}\left(\left[z^{+}, w^{+}\right]\right)-\mathcal{A}_{F}\left(\left[z^{-}, w^{-}\right]\right) \\
& +\int_{0}^{1} \max _{x, s}\left|\frac{\partial H_{t}^{s}}{\partial s}\right| d t .
\end{aligned}
$$

Proof. The proof is an immediate consequence of (5.8) and omitted.

Here we would like to emphasize that the upper estimates (5.10)-(5.11) or (5.13)-(5.14) do not depend on the choice of $j$ or of $\rho$, but depend only on the homotopy $\mathcal{H}$ itself.

Motivated by the upper estimate (5.10), we introduce the following definition

Definition 5.4. Let $\mathcal{H}=\{H(s)\}_{0 \leq s \leq 1}$ be a homotopy of Hamiltonians. We define the negative part of the variation and the positive part of the variation of $\mathcal{H}$ by

$$
\begin{aligned}
E^{-}(\mathcal{H}) & :=\int_{0}^{1}-\min _{x, s}\left(\frac{\partial H_{t}^{s}}{\partial s}\right) d t . \\
E^{+}(\mathcal{H}) & :=\int_{0}^{1} \max _{x, s}\left(\frac{\partial H_{t}^{s}}{\partial s}\right) d t .
\end{aligned}
$$

And we define the total variation $E(\mathcal{H})$ of $\mathcal{H}$ by

$$
E(\mathcal{H})=E^{-}(\mathcal{H})+E^{+}(\mathcal{H})
$$

If we denote by $\mathcal{H}^{-1}$ the time reversal of $\mathcal{H}$, i.e., the homotopy given by

$$
\mathcal{H}^{-1}: s \in[0,1] \mapsto H^{1-s},
$$

then we have the identity

$$
E^{ \pm}\left(\mathcal{H}^{-1}\right)=E^{\mp}(\mathcal{H}) \quad \text { and } E\left(\mathcal{H}^{-1}\right)=E(\mathcal{H}) .
$$

With these definitions, applied to a pair $(\mathcal{H}, j)$ such that their ends $H(0)$ and $H(1)$ are nondegenerate, the a priori energy estimate (5.11) can be written as

$$
\int\left|\frac{\partial u}{\partial \tau}\right|_{J \rho(\tau)}^{2} \leq-\mathcal{A}_{F}\left(\left[z^{+}, w^{+}\right]\right)+\mathcal{A}_{H}\left(\left[z^{-}, w^{-}\right]\right)+E^{-}(\mathcal{H})
$$

for a monotone $\rho$, and (5.14) as

$$
\int\left|\frac{\partial u}{\partial \tau}\right|_{J \rho(\tau)}^{2} \leq-\mathcal{A}_{F}\left(\left[z^{+}, w^{+}\right]\right)+\mathcal{A}_{H}\left(\left[z^{-}, w^{-}\right]\right)+E(\mathcal{H})
$$

for a general $\rho$.

Note that when $\mathcal{H}$ is the linear homotopy

$$
\mathcal{H}^{l i n}: s \mapsto(1-s) H_{1}+s H_{2}
$$


between $H_{1}$ and $H_{2}, E^{ \pm}(\mathcal{H})$ and $E(\mathcal{H})$ just become $E^{ \pm}\left(H_{2}-H_{1}\right)$, and $\| H_{2}-$ $H_{1} \|$, respectively: We recall the definitions

$$
\begin{aligned}
E^{-}(H) & =\int_{0}^{1}-\min _{x} H_{t} d t, \quad E^{+}(H)=\int_{0}^{1} \max _{x} H_{t} d t \\
\|H\| & =E^{+}(H)+E^{-}(H)=\int_{0}^{1}\left(\max _{x} H_{t}-\min _{x} H_{t}\right) d t .
\end{aligned}
$$

Therefore, taking the infimum of $E(\mathcal{H})$ over all $\mathcal{H}$ with fixed end points $H(0)=$ $H_{0}$ and $H(1)=H_{1}$, we have the inequality

$$
\inf _{\mathcal{H}}\left\{E(\mathcal{H}) \mid H(0)=H_{0}, H(1)=H_{1}\right\} \leq\left\|H_{1}-H_{0}\right\|
$$

which is a strict inequality in general. It seems to be an interesting problem to investigate the geometric meaning of the quantity in the left hand side. This will be a subject of the future study.

Next, we consider the triple

$$
\left(H_{\alpha}, H_{\beta}, H_{\gamma}\right)
$$

of Hamiltonians and homotopies $\mathcal{H}_{1}, \mathcal{H}_{2}$ connecting from $H_{\alpha}$ to $H_{\beta}$ and $H_{\beta}$ to $H_{\gamma}$ respectively. We define their concatenation $\mathcal{H}_{1} \# \mathcal{H}_{2}=\left\{H_{3}(s)\right\}_{1 \leq s \leq 1}$ by

$$
H_{3}(s)= \begin{cases}H_{1}(2 s) & 0 \leq s \leq \frac{1}{2} \\ H_{2}(2 s-1) & \frac{1}{2} \leq s \leq 1\end{cases}
$$

From the definition of $E^{ \pm}$and $E$ for the homotopy $\mathcal{H}$ above, we immediately have the following lemma

Lemma 5.5. All $E^{ \pm}$and $E$ are additive under the concatenation of homotopies. In other words, for any triple $\left(H_{\alpha}, H_{\beta}, H_{\gamma}\right)$ and homotopies $\mathcal{H}_{1}, \mathcal{H}_{2}$ as above, we have

$$
E^{ \pm}\left(\mathcal{H}_{1} \# \mathcal{H}_{2}\right)=E^{ \pm}\left(\mathcal{H}_{1}\right)+E^{ \pm}\left(\mathcal{H}_{2}\right)
$$

The same additivity holds for $E$.

Next we note that due to the choice of the cut-off function $\rho$, the continuity equation (5.3) is autonomous for the region $|\tau|>R$, i.e., is invariant under the translation by $\tau$. When we are given a triple $\left(H_{\alpha}, H_{\beta}, H_{\gamma}\right)$, this fact enables us to glue solutions of two such equations corresponding to the pairs $\left(H_{\alpha}, H_{\beta}\right)$ and $\left(H_{\beta}, H_{\gamma}\right)$ respectively.

Now a more precise explanation is in order. For a given pair of cut-off functions

$$
\rho=\left(\rho_{1}, \rho_{2}\right)
$$

and a positive number $R>0$, we define an elongated homotopy of $\mathcal{H}_{1} \# \mathcal{H}_{2}$

$$
\mathcal{H}_{1} \#(\rho ; R) \mathcal{H}_{2}=\left\{H_{(\rho ; R)}(\tau)\right\}_{-\infty<\tau<\infty}
$$


by

$$
H_{(\rho ; R)}(\tau, t, x)= \begin{cases}H_{1}\left(\rho_{1}(\tau+2 R), t, x\right) & \tau \leq 0 \\ H_{2}\left(\rho_{2}(\tau-2 R), t, x\right) & \tau \geq 0\end{cases}
$$

Note that

$$
H_{(\rho ; R)} \equiv \begin{cases}H_{\alpha} & \text { for } \tau \leq-\left(R_{1}+2 R\right) \\ H_{\beta} & \text { for }-R \leq \tau \leq R \\ H_{\gamma} & \text { for } \tau \geq R_{2}+2 R\end{cases}
$$

for some sufficiently large $R_{1}, R_{2}>0$ depending on the cut-off functions $\rho_{1}, \rho_{2}$ and the homotopies $\mathcal{H}_{1}, \mathcal{H}_{2}$ respectively. In particular this elongated homotopy is always smooth, even when the usual glued homotopy $\mathcal{H}_{1} \# \mathcal{H}_{2}$ may not be so. We define the elongated homotopy $j_{1} \#(\rho ; R) j_{2}$ of $j_{1} \# j_{2}$ in a similar way.

For an elongated homotopy $\left(j_{1} \#_{(\rho ; R)} j_{2}, \mathcal{H}_{1} \#_{(\rho, R)} \mathcal{H}_{2}\right)$, we consider the associated perturbed Cauchy-Riemann equation

$$
\left\{\begin{array}{l}
\frac{\partial u}{\partial \tau}+J_{3}^{\rho(\tau)}\left(\frac{\partial u}{\partial t}-X_{H_{3}^{\rho(\tau)}}(u)\right)=0 \\
\lim _{\tau \rightarrow-\infty} u(\tau)=z^{-}, \lim _{\tau \rightarrow \infty} u(\tau)=z^{+}
\end{array}\right.
$$

with the condition (5.4). The following lemma will be used later.

Lemma 5.6. Let $H_{\alpha}, H_{\beta}, H_{\gamma}$ be given, and let $\mathcal{H}_{i}$ for $i=1,2$ be homotopies between them respectively. Fix a generic homotopies $j_{i}$ for $i=1,2$. For any given pair $\rho=\left(\rho_{1}, \rho_{2}\right)$ of monotone cut-off functions and a positive number $R>0$, we consider the elongated homotopy

$$
j_{1} \#_{(\rho ; R)} j_{2}=\left\{J_{(\rho ; R)}(\tau)\right\}_{\tau \in \mathbb{R}}, \quad \mathcal{H}_{1} \#_{(\rho ; R)} \mathcal{H}_{2}=\left\{H_{(\rho ; R)}(\tau)\right\}_{\tau \in \mathbb{R}}
$$

defined as above, and the associated equation (5.19). Then for any finite energy solution of (5.19), we have the inequality

(5.20) $\mathcal{A}_{H_{\gamma}}(u(\infty))-\mathcal{A}_{H_{\alpha}}(u(-\infty)) \leq-\int\left|\frac{\partial u}{\partial \tau}\right|_{J_{(\rho ; R)}(\tau)}^{2}+\left(E^{-}\left(\mathcal{H}_{1}\right)+E^{-}\left(\mathcal{H}_{2}\right)\right)$.

This can be rewritten also as an a priori energy bound

$$
\begin{aligned}
\int\left|\frac{\partial u}{\partial \tau}\right|_{J_{(\rho ; R)}(\tau)}^{2} \leq- & \mathcal{A}_{H_{\gamma}}(u(\infty))+\mathcal{A}_{H_{\alpha}}(u(-\infty)) \\
& +\left(E^{-}\left(\mathcal{H}_{1}\right)+E^{-}\left(\mathcal{H}_{2}\right)\right) .
\end{aligned}
$$

Proof. This is a special case of Lemma 5.2 because

$$
E^{-}\left(\mathcal{H}_{1} \# \mathcal{H}_{2}\right)=E^{-}\left(\mathcal{H}_{1}\right)+E^{-}\left(\mathcal{H}_{2}\right)
$$

from Lemma 5.5, and $\mathcal{H}_{1} \#(\rho ; R) \mathcal{H}_{2}=\left\{H_{(\rho ; R)}(\tau)\right\}$ itself is an elongation of the homotopy $\mathcal{H}_{1} \# \mathcal{H}_{2}$ corresponding to the cut-off function

$$
\rho(\tau)= \begin{cases}\frac{1}{2} \rho_{1}(\tau+2 R) & \tau \leq 0 \\ \frac{1}{2}+\frac{1}{2} \rho_{2}(\tau-2 R) & \tau \geq 0\end{cases}
$$


which remains monotone when $\rho_{1}, \rho_{2}$ are monotone.

Now let $u_{1}$ and $u_{2}$ be given solutions of (5.3)-(5.4) associated to $\rho_{1}$ and $\rho_{2}$ respectively. If we define the pre-gluing map $u_{1} \#_{R} u_{2}$ by the formula

$$
u_{1} \#_{R} u_{2}(\tau, t)= \begin{cases}u_{1}(\tau+2 R, t) & \text { for } \tau \leq-R \\ u_{2}(\tau-2 R, t) & \text { for } \tau \geq R\end{cases}
$$

and a suitable interpolation between them by a partition of unity on the region $-R \leq \tau \leq R$, the assignment defines a diffeomorphism

$$
\left(u_{1}, u_{2}, R\right) \rightarrow u_{1} \#_{R} u_{2}
$$

from

$$
\mathcal{M}\left(j_{1}, \mathcal{H}_{1} ;\left[z_{1}, w_{1}\right],\left[z_{2}, w_{2}\right]\right) \times \mathcal{M}\left(j_{2}, \mathcal{H}_{2} ;\left[z_{2}, w_{2}\right],\left[z_{3}, w_{3}\right]\right) \times\left(R_{0}, \infty\right)
$$

onto its image, provided $R_{0}$ is sufficiently large. Denote by $\bar{\partial}_{(\mathcal{H}, j ; \rho)}$ the corresponding perturbed Cauchy-Riemann operator

$$
u \mapsto \frac{\partial u}{\partial \tau}+J_{3}^{\rho(\tau)}\left(\frac{\partial u}{\partial t}-X_{H_{3}^{\rho(\tau)}}(u)\right)
$$

acting on the maps $u$ satisfying the asymptotic condition imposed in (5.19) and fixed homotopy condition $[u]=C \in \pi_{2}\left(z^{-}, z^{+}\right)$. By perturbing $u_{1} \#_{R} u_{2}$ by the amount that is smaller than the error for $u_{1} \#{ }_{R} u_{2}$ to be a genuine solution, i.e., less than a weighted $L^{p}$-norm, for $p>2$,

$$
\left\|\bar{\partial}_{(\mathcal{H}, j ; \rho)}\left(u_{1} \#(\rho ; R) u_{2}\right)\right\|_{p}
$$

in a suitable weighted $W^{1, p}$ space of $u$ 's (see [5, 6]), one can construct a unique genuine solution near $u_{1} \#_{R} u_{2}$. By an abuse of notation, we will denote this genuine solution also by $u_{1} \#_{R} u_{2}$. Then the corresponding map defines an embedding

$$
\begin{aligned}
& \mathcal{M}\left(j_{1}, \mathcal{H}_{1} ;\left[z_{1}, w_{1}\right],\left[z_{2}, w_{2}\right]\right) \times \mathcal{M}\left(j_{2}, \mathcal{H}_{2} ;\left[z_{2}, w_{2}\right],\left[z_{3}, w_{3}\right]\right) \times\left(R_{0}, \infty\right) \rightarrow \\
& \rightarrow \mathcal{M}\left(j_{1} \#(\rho ; R) j_{2}, \mathcal{H}_{1} \#(\rho ; R)\right. \\
&\left.\mathcal{H}_{2} ;\left[z_{1}, w_{1}\right],\left[z_{3}, w_{3}\right]\right) .
\end{aligned}
$$

Especially when we have

$$
\mu_{H_{\beta}}\left(\left[z_{2}, w_{2}\right]\right)-\mu_{H_{\alpha}}\left(\left[z_{1}, w_{1}\right]\right)=\mu_{H_{\gamma}}\left(\left[z_{3}, w_{3}\right]\right)-\mu_{H_{\beta}}\left(\left[z_{2}, w_{2}\right]\right)=0
$$

both $\mathcal{M}\left(j_{1}, \mathcal{H}_{1} ;\left[z_{1}, w_{1}\right],\left[z_{2}, w_{2}\right]\right)$ and $\mathcal{M}\left(j_{2}, \mathcal{H}_{2} ;\left[z_{2}, w_{2}\right],\left[z_{3}, w_{3}\right]\right)$ are compact, and so consist of a finite number of points. Furthermore the image of the above mentioned embedding exhausts the 'end' of the

$$
\mathcal{M}\left(j_{1} \#_{(\rho ; R)} j_{2}, \mathcal{H}_{1} \#_{(\rho ; R)} \mathcal{H}_{2} ;\left[z_{1}, w_{1}\right],\left[z_{3}, w_{3}\right]\right)
$$

and the boundary of its compactification consists of the broken trajectories

$$
u_{1} \#(\rho ; \infty) u_{2}=u_{1} \# \infty u_{2} \text {. }
$$


This then proves the gluing identity

$$
h_{\mathcal{H}_{1} \#(\rho ; R)} \mathcal{H}_{2}=h_{\left(\mathcal{H}_{1} ; \rho_{1}\right)} \circ h_{\left(\mathcal{H}_{2} ; \rho_{2}\right)}
$$

(modulo any filtration order we want) for a sufficiently large $R>0$, suppressing the $j$-dependence. Here we remind the readers that the homotopy $\mathcal{H}_{1} \#(\rho ; R) \mathcal{H}_{2}$ itself is an elongated homotopy of the glued homotopy $\mathcal{H}_{1} \# \mathcal{H}_{2}$.

Now we study the chain map $h_{(\mathcal{H}, j ; \rho)}$ more closely, and compare the maps for two different choices of the cut-off function $\rho$ in relation to the level changes of the transferred Floer cycles. Suppose that $\rho_{1}$ and $\rho_{2}$ are two cut-off functions. We choose a one-parameter family

$$
\kappa \mapsto[0,1] \rightarrow(1-\kappa) \rho_{1}+\kappa \rho_{2}:=\rho(\kappa)
$$

and consider the homotopy of homotopies of Hamiltonians

$$
(\kappa, \tau) \mapsto H(\rho(\kappa)(\tau), \cdot, \cdot) .
$$

By considering the family $\bar{j}$ and $\overline{\mathcal{H}}$ associated to this homotopy, the chain homotopy map $H_{(\bar{j}, \overline{\mathcal{H}} ; \bar{\rho})}$ is constructed by counting solutions of (5.3) at nonregular, but parametrically regular parameters $\kappa$. We will elaborate this remark in the proof of the following main result in this section.

Proposition 5.7. Let $(\mathcal{H}, j)$ be a given homotopy between $\left(H^{0}, J^{0}\right)$ and $\left(H^{1}, J^{1}\right)$. Let $\rho_{1}, \rho_{2}$ be two cut-off functions and $\bar{\rho}$ be the homotopy (5.24) between them. Let $(\bar{j}, \overline{\mathcal{H}})$ be the associated homotopy of homotopies over $\kappa \in[0,1]$. Then we have

$$
h_{\left(\mathcal{H}, j ; \rho_{1}\right)}-h_{\left(\mathcal{H}, j ; \rho_{2}\right)}=\partial_{\left(J^{1}, H^{1}\right)} \circ H_{(\overline{\mathcal{H}}, \bar{j} ; \bar{\rho})}+H_{(\overline{\mathcal{H}}, \bar{j} ; \bar{\rho})} \circ \partial_{\left(J^{0}, H^{0}\right)} .
$$

Furthermore the inequality

$$
\lambda_{H^{1}}\left(H_{(\overline{\mathcal{H}}, \bar{j} ; \bar{\rho})}(\alpha)\right) \leq \lambda_{H^{0}}(\alpha)+E(\mathcal{H})
$$

holds for any Floer cycle $\alpha$ of $\left(J^{0}, H^{0}\right)$.

Proof. It remains to prove (5.25). The map $H_{(\bar{j}, \overline{\mathcal{H}} ; \bar{\rho})}$ is determined by the number of the pairs

$$
(\kappa, u)
$$

(See [8]), where $\kappa \in(0,1)$ and $u$ is a solution of

$$
\left\{\begin{array}{l}
\frac{\partial u}{\partial \tau}+J^{\rho_{\kappa}}(\tau)\left(\frac{\partial u}{\partial t}-X_{H^{\rho_{\kappa}(\tau)}}(u)\right)=0 \\
\lim _{\tau \rightarrow-\infty} u(\tau)=z^{-}, \lim _{\tau \rightarrow \infty} u(\tau)=z^{+}
\end{array}\right.
$$

satisfying

$$
w^{+} \sim w^{-} \# u ; \quad \mu_{\mathcal{H}_{\kappa}}([u])=-1 .
$$

Therefore we can estimate the level change

$$
\mathcal{A}_{\left(H_{\beta}, J_{\beta}\right)}\left(\left[z^{+}, w^{+}\right]\right)-\mathcal{A}_{\left(H_{\alpha}, J_{\alpha}\right)}\left(\left[z^{-}, w^{-}\right]\right)
$$

applying (5.8) for the function $\rho=\rho_{k}=(1-\kappa) \rho_{1}+\kappa \rho_{2}$. Once we have this, (5.25) follows in the same way (5.16) was derived. This finishes the proof. 


\section{$\S 6$. Structure of Novikov Floer cycles in a Cerf family}

In this section, we prove a general structure theorem, Theorem 6.9, of Novikov Floer cycles for any Cerf homotopy $\mathcal{H}=\{H(\eta)\}$ of Hamiltonian functions.

Let $H$ be any one-periodic Hamiltonian and consider the perturbed CauchyRiemann equation

$$
\frac{\partial u}{\partial \tau}+J\left(\frac{\partial u}{\partial t}-X_{H}(u)\right)=0
$$

for a generic $J$. We call a solution $u$ stationary if it is $\tau$-independent. We define

$$
A_{(J, H)}:=\inf \left\{\int\left|\frac{\partial u}{\partial \tau}\right|_{J}^{2} \mid u \text { satisfies (6.1) and is not stationary }\right\} .
$$

The positivity of $A_{(J, H)}$ is an easy consequence of the Gromov compactness type theorem (see [19] for details of such a proof).

We also introduce the following invariant of a compact family $K \subset \mathcal{J}_{\omega}$ of compatible almost complex structures. Let

$$
K ;[0,1]^{n} \rightarrow \mathcal{J}_{\omega}
$$

be a $n$-parameter family in the $C^{1}$-topology, and define $A(\omega ; K)$ be the constant

$$
A(\omega ; K)=\inf _{\kappa \in[0,1]^{n}}\{A(\omega, J(\kappa))\} .
$$

This is always positive (see [19] for the proof in a similar context), and enjoys the following lower semi-continuity property.

Proposition 6.1. $A(\omega ; K)$ is lower semi-continuous in $K$. In other words, for any given $K$ and $0<\epsilon<A(\omega ; K)$, there exists some $\delta=\delta(K, \epsilon)>0$ such that for any $K^{\prime}$ with $\left\|K^{\prime}-K\right\|_{C^{1}} \leq \delta$ we have

$$
A\left(\omega ; K^{\prime}\right) \geq A(\omega ; K)-\epsilon .
$$

Proof. The arguments used in the proof of this proposition is similar to the one used in [19, Section 4].

Suppose to the contrary that there exists some $0<\epsilon<A(\omega ; K)$ for which there are sequences $\delta_{k} \rightarrow 0, K_{k}$ with $\left\|K_{k}-K\right\|_{C^{1}} \leq \delta_{k}$ and

$$
A\left(\omega ; K_{k}\right)<A(\omega ; K)-\epsilon
$$

for all $k$. By the definition of $A\left(\omega ; K_{k}\right)$, this implies that there exist nonconstant $J_{k, t_{k}}$-holomorphic spheres $w_{k}$ for $t_{k} \in[0,1]$ such that we have

$$
0<E_{J_{k, t_{k}}}\left(w_{k}\right)<A(\omega ; K)-\epsilon .
$$


By choosing a subsequence, we may assume that $t_{k} \rightarrow t_{\infty} \in[0,1]$ and $J_{k, t_{k}} \rightarrow$ $J_{t_{\infty}}$ in the $C^{1}$-topology. By the energy bound (6.3) for $w_{k}$ and since $K_{k} \rightarrow K$ in the $C^{1}$ topology, we can produce a $J_{\infty}$-holomorphic map

$$
w_{\infty}=\sum_{\ell} w_{\infty, \ell}
$$

whose total energy satisfies

$$
E_{J_{t_{\infty}}}\left(w_{\infty}\right) \leq A(\omega ; K)-\epsilon
$$

Then by definition of $A(\omega ; K),(6.4)$ implies all the components of $w_{\infty}$ must be constant. By choosing a subsequence if necessary, it follows that $w_{k}$ converges to a constant map, say $p \in M$, in the $C^{1}$-topology, and also

$$
\lim _{k \rightarrow \infty} E_{J_{k, t_{k}}}\left(w_{k}\right)=0 .
$$

In particular, the image of $w_{k}$ is contained in a (contractible) Darboux neighborhood $U$ of $p$ and so we have

$$
\int w_{k}^{*} \omega=0
$$

On the other hand, by the compatibility of $J_{k, t}$ to $\omega$, we also have

$$
E_{J_{k, t_{k}}}\left(w_{k}\right)=\int w_{k}^{*} \omega
$$

which in turn implies $E_{J_{k, t_{k}}}\left(w_{k}\right)=0$ for all sufficiently large $k$. But this contradicts the assumption that $w_{k}$ are nonconstant. This finishes the proof.

By a similar argument, we prove the following proposition.

Proposition 6.2. Let $(J, H)$ be a pair with $H$ nondegenerate. Then for any given $0<\epsilon<\min \left\{A_{(J, H)}, A(\omega ; J)\right\}$, there exists some $\delta_{2}=\delta_{2}(J, H, \epsilon)>0$ such that for any $\left(J^{\prime}, H^{\prime}\right)$ with $\left\|\left(J^{\prime}, H^{\prime}\right)-(J, H)\right\|_{C^{\infty}} \leq \delta$ we have

$$
A_{\left(J^{\prime}, H^{\prime}\right)} \geq \min \left\{A_{(J, H)}, A(\omega ; J)\right\}-\epsilon .
$$

Proof. Suppose the contrary that there exists some $0<e<\min \left\{A_{(J, H)}, A(\omega ; J)\right\}$ for which there are sequences $\delta_{k} \rightarrow 0,\left(J_{k}, H_{k}\right)$ with $\left\|\left(J_{k}, H_{k}\right)-(J, H)\right\|_{C} \leq \delta_{k}$ and

$$
A_{\left(J_{k}, H_{k}\right)}<\min \left\{A_{(J, H)}, A(\omega ; J)\right\}-\epsilon
$$

for all $k$. By the definition of $A_{(J, H)}$, this implies that there exist solutions $u_{k}$ of $(6.1)$ for $\left(J_{k}, H_{k}\right)$ such that we have

$$
0<E_{\left(J_{k}, H_{k}\right)}\left(u_{k}\right)<\min \left\{A_{(J, H)}, A(\omega ; J)\right\}-\epsilon .
$$


By the energy bound $(6.5)$ for $u_{k}$ and since $\left(J_{k}, H_{k}\right) \rightarrow(J, H)$ in the $C^{\infty}$ topology, we can produce a cusp-trajectory

$$
u_{\infty}=\sum_{\ell} u_{\infty, \ell}
$$

of (6.1) for $(J, H)$ with

$$
E_{(J, H)}\left(u_{\infty}\right) \leq \min \left\{A_{(J, H)}, A(\omega ; J)\right\}-\epsilon .
$$

Therefore by the definitions of $A_{(J, H)}$ and $A(\omega ; J)$, it follows that all the summand $u_{\infty, \ell}$ in $u_{\infty}$ must be trivial in that all the principal components are stationary and all the bubble components are constant. In particular, we have $E_{(J, H)}\left(u_{\infty}\right)=0$ and $u_{k}$ uniformly converges to a periodic orbit $z_{\infty}$ of $H$ in the (fine) $C^{\infty}$ topology. These then imply

$$
\lim _{k \rightarrow \infty} E_{\left(J_{k}, H_{k}\right)}\left(u_{k}\right)=0 .
$$

On the other hand, since we assume that $H$ is nondegenerate and since we can make $H_{k}$ arbitrarily $C^{\infty}$-close to $H$, by taking sufficiently large $k, H_{k}$ are nondegenerate and there is a canonical one-one correspondence between $\operatorname{Per}(H)$ and $\operatorname{Per}\left(H_{k}\right)$ for each $k$. In particular, there exists some $k_{0} \in \mathbb{Z}_{+}$and $c_{1}>0$ such that

$$
d_{C^{0}}\left(z, z^{\prime}\right) \geq c_{1}
$$

for any periodic orbits $z \neq z^{\prime}$ of $H_{k}$ with $k \geq k_{0}$. Since $J_{k} \rightarrow J$ in the $C^{\infty}$ topology, this and (6.6) imply that all $u_{k}$ must satisfy

$$
u_{k}(-\infty)=u_{k}(\infty):
$$

For otherwise it would imply that there exists $c_{2}>0$ independent of $k$ such that $E_{\left(J_{k}, H_{k}\right)}\left(u_{k}\right)>c_{2}$ (See Lemma A.3 for the proof of this statement), which would contradict (6.6).

We now recall the following lemma from [19, Lemma 4.5].

Lemma 6.3. Suppose that $u: \mathbb{R} \times S^{1} \rightarrow M$ is any finite energy solution of

that satisfies

$$
\left\{\begin{array}{l}
\frac{\partial u}{\partial \tau}+J\left(\frac{\partial u}{\partial t}-X_{H}(u)\right)=0 \\
E_{J}(u)=\int\left|\frac{\partial u}{\partial \tau}\right|_{J_{t}}^{2}<\infty
\end{array}\right.
$$

$$
u(-\infty, t)=u(\infty, t) .
$$

Then $\int_{\mathbb{R} \times S^{1}} u^{*} \omega$ converges, and we have

$$
E_{J}(u)=\int_{\mathbb{R} \times S^{1}} u^{*} \omega .
$$

Going back to our proof of Proposition 6.2, (6.7) and this lemma imply

$$
E_{\left(J_{k}, H_{k}\right)}\left(u_{k}\right)=\int u_{k}^{*} \omega
$$


On the other hand, since $u_{k}$ satisfies (6.7) and converges to a periodic orbit $z_{\infty}$ in the (fine) $C^{\infty}$ topology, it defines a cycle homologous to the one dimensional cycle $z_{\infty}$ and hence $\int u_{k}^{*} \omega=0$. In turn (6.8) implies we must have

$$
E_{\left(J_{k}, H_{k}\right)}\left(u_{k}\right)=0
$$

which contradicts the assumption that $u_{k}$ are non-stationary. This finishes the proof.

For a choice of $(\mathcal{H}, j)$ with $\mathcal{H} \in \mathcal{P}^{\operatorname{Cerf}}\left(\mathcal{H}_{m}(M) ; H_{1}, H_{2}\right)$, we define

$$
\begin{aligned}
\mathcal{N} t(\mathcal{H}, j)=\{\eta \in[0,1] \backslash \operatorname{Sing}(\mathcal{H}) \mid \text { the pair }(J(\eta), H(\eta)) \\
\text { has a solution of }(4.3) \text { with the Fredholm index } 0\} .
\end{aligned}
$$

The following proposition was proved in Section 4 (Theorem 4.6). We rephrase Theorem 4.6 (1) to manifest what the transversality means in a more concrete context. It provides a structure theorem of $\mathcal{N} t(\mathcal{H}, j)$ for a generic choice of $j$ for a given homotopy $\mathcal{H}$. We will consider only the case, where $\mathcal{H}$ is a Cerf homotopy in the statement.

Proposition \& Definition 6.4. Let $\mathcal{H}$ be a Cerf homotopy and $\mathcal{N} t(\mathcal{H}, j)$ be as above. Then for a generic choice of the homotopy $j$ of almost complex structures, the followings hold:

(1) At each $\eta \in \mathcal{N} t(\mathcal{H}, j)$, the equation (4.3)-(4.4) for $(J, H)=(J(\eta), H(\eta))$ has exactly one pair $z^{+}, z^{-} \in \operatorname{Per}(H(\eta))$, and one non-trivial solution u connecting $z^{ \pm}$which has Fredholm index 0.

(2) Write $\mathcal{N} t(\mathcal{H}, j)$ as an increasing union

$$
\mathcal{N} t(\mathcal{H}, j)=\bigcup_{N=1}^{\infty} \mathcal{N} t_{N}(\mathcal{H}, j)
$$

where we define

$$
\begin{array}{r}
\mathcal{N} t_{N}(\mathcal{H}, j)=\{\eta \in \mathcal{N} t(\mathcal{H}, j) \mid \text { the unique solution } u \\
\text { satisfies } \left.E_{(J(\eta), H(\eta))} \leq N\right\} .
\end{array}
$$

Then $\mathcal{N} t_{N}(\mathcal{H}, j)$ is a compact zero dimensional manifold for each given $N \in \mathbb{Z}_{+}$and in particular a finite subset of $[0,1]$. In particular, there are only countably many such points $\eta \in[0,1] \backslash \operatorname{Sing}(\mathcal{H})$.

We call a corresponding homotopy $(\mathcal{H}, j)$ a Floer homotopy and call any point $\eta$ lying in $[0,1] \backslash(\mathcal{S i n g}(\mathcal{H}) \cup \mathcal{N} t(\mathcal{H}, j))$ a Floer point of the Floer homotopy $(\mathcal{H}, j)$.

For a Floer homotopy $(\mathcal{H}, j)$, the Floer homology $H F_{*}(H(\eta), J(\eta))$ is welldefined at any Floer point $\eta$. Furthermore the subset $\operatorname{Sing}(\mathcal{H}) \cup \mathcal{N} t(\mathcal{H}, j)$ is at most countable and so the subset $[0,1] \backslash(\mathcal{S i n g}(\mathcal{H}) \cup \mathcal{N} t(\mathcal{H}, j))$ of Floer points in $[0,1]$ is residual and dense. 
Next we compare the levels of Novikov Floer cycles and of their boundaries. Suppose that $\eta, \eta^{\prime}$ are sufficiently close so that the associated pairs are defined. Let $\left(z, z^{\prime}\right)$ be an associated pair of $\left(H(\eta), H\left(\eta^{\prime}\right)\right)$. We denote by

$$
\mathcal{M}_{\eta \eta^{\prime}}^{\rho}\left(z, z^{\prime} ;\left[u_{z z^{\prime}}^{c a n}\right]\right):=\mathcal{M}\left(\left(\mathcal{H}_{\eta \eta^{\prime}}, j_{\eta \eta^{\prime}} ; \rho\right) ; z, z^{\prime}:\left[u_{z z^{\prime}}^{c a n}\right]\right)
$$

the set of solutions $u$ of (5.3)-(5.4) homotopic to the canonical cylinder $u_{z z^{\prime}}^{c a n}$ relative to the boundary, and satisfying

$$
u(-\infty)=z \in \operatorname{Per}(H(\eta)), \quad u(\infty)=z^{\prime} \in \operatorname{Per}\left(H\left(\eta^{\prime}\right)\right) .
$$

A straightforward calculation using (5.9) gives rise to the following proposition whose proof we leave to the readers.

Proposition 6.5. Let $F$ and $H$ be nondegenerate Hamiltonians and $\mathcal{H}$ be a homotopy between them. Fix any, monotone or not, cut-off function $\rho$. Let $u_{1}, u_{2}$ be solutions satisfying (5.3) with fixed asymptotic conditions

$$
u_{i}(-\infty)=\left[z^{-}, w^{-}\right], \quad u_{i}(\infty)=\left[z^{+}, w^{+}\right]
$$

for some $w^{-}$and be homologous to each other in that the compactified torus $u_{1} \# \bar{u}_{2}$ satisfies

$$
u_{1} \# \bar{u}_{2} \sim 0
$$

Then we have

$$
\left|E_{(\mathcal{H}, j ; \rho)}\left(u_{1}\right)-E_{(\mathcal{H}, j ; \rho)}\left(u_{2}\right)\right| \leq E(\mathcal{H}) .
$$

In particular, when $\mathcal{H} \equiv H, j \equiv J$, we have

$$
E_{(J, H)}\left(u_{1}\right)=E_{(J, H)}\left(u_{2}\right) .
$$

Let $\eta \in[0,1]$ be a point for which $H(\eta)$ is nondegenerate. We then introduce the following function

$$
\begin{aligned}
\ell_{(\mathcal{H}, j)}^{\rho}\left(\eta, \eta^{\prime}\right):=\max _{z, z^{\prime}, u}\left\{\int\left|\frac{\partial u}{\partial \tau}\right|_{J^{\rho(\tau)}}^{2} \mid\right. & u \in \mathcal{M}_{\eta \eta^{\prime}}^{\rho}\left(z, z^{\prime} ;\left[u_{z z^{\prime}}^{c a n}\right]\right), \\
& \left.\left(z, z^{\prime}\right) \text { is an associated pair }\right\}
\end{aligned}
$$

defined for $\eta^{\prime}$ such that $\left|\eta-\eta^{\prime}\right|$ is sufficiently small and $H\left(\eta^{\prime}\right)$ is nondegenerate.

And for a given $\eta_{\infty} \in \mathcal{S} \operatorname{sing}(\mathcal{H})$, we consider the bifurcation pair $\left(z^{+}(\eta)\right.$, $\left.z^{-}(\eta)\right)$ for the pair $(J(\eta), H(\eta))$ as $\eta \rightarrow \eta_{\infty} \in \mathcal{S i n g}(\mathcal{H})$. We define the function

$$
\ell_{\left(\mathcal{H}, j ; \eta_{\infty}\right)}(\eta):=\max _{u}\left\{\int\left|\frac{\partial u}{\partial \tau}\right|_{J(\eta)}^{2} \mid u \in \mathcal{M}\left(z^{+}(\eta), z^{-}(\eta) ;\left[u^{c a n}\right]\right)\right\}
$$

defined for $\eta$ with $\left|\eta-\eta_{\infty}\right|$ sufficiently small, where

$$
\mathcal{M}\left(z^{+}(\eta), z^{-}(\eta) ;\left[u^{c a n}\right]\right):=\mathcal{M}\left(H(\eta), J(\eta) ; z^{+}(\eta), z^{-}(\eta) ;\left[u^{c a n}\right]\right) .
$$

Proposition 6.6. Let $\eta, \eta^{\prime}$ be nondegenerate points such that $\left|\eta-\eta^{\prime}\right|$ is sufficiently small and $\left(z, z^{\prime}\right)$ be an associated pair such that $\mathcal{M}_{\eta \eta^{\prime}}^{\rho_{1}}\left(z, z^{\prime} ;\left[u_{z z^{\prime}}^{c a n}\right]\right)$ is 
nonempty for a cut-off function $\rho_{1}$. Then there exists a function $C_{(\mathcal{H}, j)}(r)$ depending only on $(\mathcal{H}, j)$ such that

(1) $C_{(\mathcal{H}, j)}(r) \rightarrow 0$ as $r \rightarrow 0$ and

(2) $\ell_{(\mathcal{H}, j)}^{\rho}\left(\eta, \eta^{\prime}\right) \leq C_{(\mathcal{H}, j)}\left(\left|\eta-\eta^{\prime}\right|\right)$.

Similar estimate also holds for the function $\ell_{\left(\mathcal{H}, j ; \eta_{\infty}\right)}$ uniformly over $\eta_{\infty} \in$ $\operatorname{Sing}(\mathcal{H})$.

Proof. Let $u \in \mathcal{M}_{\eta \eta^{\prime}}\left(z, z^{\prime} ;\left[u_{z z^{\prime}}^{c a n}\right]\right)$. Using the fact that $u$ is a solution of (5.3), we derive

$$
\int\left|\frac{\partial u}{\partial \tau}\right|_{J^{\rho_{1}(\tau)}}^{2} \leq-\mathcal{A}_{H\left(\eta^{\prime}\right)}\left(\left[z^{\prime}, w \# u^{c a n}\right]\right)+\mathcal{A}_{H(\eta)}([z, w])+E\left(\mathcal{H}_{\eta \eta^{\prime}}\right)
$$

from (5.16). On the other hand, from the definition of the action functional, we have

$$
\begin{aligned}
\mathcal{A}_{H\left(\eta^{\prime}\right)}\left(\left[z^{\prime}, w \# u^{c a n}\right]\right) & =-\int_{w \# u^{c a n}} \omega-\int_{0}^{1} H\left(\eta^{\prime}\right)\left(t, z^{\prime}(t)\right) d t \\
\mathcal{A}_{H(\eta)}([z, w]) & =-\int_{w} \omega-\int_{0}^{1} H(\eta)(t, z(t)) d t
\end{aligned}
$$

and so we get

$$
\begin{aligned}
& \mathcal{A}_{H\left(\eta^{\prime}\right)}\left(\left[z^{\prime}, w \# u^{c a n}\right]\right)-\mathcal{A}_{H(\eta)}([z, w]) \\
= & -\int_{u^{c a n}} \omega-\int_{0}^{1}\left(H\left(\eta^{\prime}\right)\left(t, z^{\prime}(t)\right)-H(\eta)(t, z(t))\right) d t .
\end{aligned}
$$

Substituting (6.16) into (6.15), we get

$$
\int\left|\frac{\partial u}{\partial \tau}\right|_{J^{\rho_{1}(\tau)}}^{2} \leq \int_{u^{c a n}} \omega+\int_{0}^{1}\left(H\left(\eta^{\prime}\right)\left(t, z^{\prime}(t)\right)-H(\eta)(t, z(t))\right) d t+E\left(\mathcal{H}_{\eta \eta^{\prime}}\right) .
$$

This can be estimated above by

$$
\operatorname{Area}_{g}\left(u_{z z^{\prime}}^{c a n}\right)+\left|\int_{0}^{1}\left(H(\eta)\left(t, z^{\prime}(t)\right)-H(\eta)(t, z(t))\right) d t\right|+E\left(\mathcal{H}_{\eta \eta^{\prime}}\right) .
$$

On the other hand, one can easily estimate

$$
\operatorname{dist}_{C^{1}}\left(z, z^{\prime}\right) \leq \widetilde{C}_{1}\left(\left\|H\left(\eta^{\prime}\right)-H(\eta)\right\|_{C^{2}}\right)
$$

for the associated pair $\left(z, z^{\prime}\right)$ with $z \in \operatorname{Per}(H(\eta))$ and $z^{\prime} \in \operatorname{Per}\left(H\left(\eta^{\prime}\right)\right)$ by a function $\widetilde{C}_{1}(r)$ such that $\widetilde{C}_{1}(r) \rightarrow 0$ as $r \rightarrow 0$.

From the explicit expression (2.6) of the canonical thin cylinder, (6.19) immediately implies

$$
\operatorname{Area}_{g}\left(u_{z z^{\prime}}^{c a n}\right) \leq \widetilde{C}_{1}\left(\left\|H\left(\eta^{\prime}\right)-H(\eta)\right\|_{C^{2}}\right) \leq C_{1}\left(\left|\eta-\eta^{\prime}\right|\right)
$$


by another function $\widetilde{C}_{1}$ satisfying $C_{1}(r) \rightarrow 0$ as $r \rightarrow 0$. On the other hand, (6.19) obviously implies

$$
\left|\int_{0}^{1}\left(H(\eta)\left(t, z^{\prime}(t)\right)-H(\eta)(t, z(t))\right) d t\right| \leq C_{2}\left(\left|\eta-\eta^{\prime}\right|\right)
$$

for a function $C_{2}=C_{2}(r)$ with the same property.

Finally since $\mathcal{H}$ is smooth and $[0,1] \times M$ is compact, we have

$$
E\left(\mathcal{H}_{\eta \eta^{\prime}}\right) \leq C_{3}\left(\left|\eta-\eta^{\prime}\right|\right)
$$

for some positive function $C_{3}$ satisfying $C_{3}(r) \rightarrow 0$ as $r \rightarrow 0$. Now, defining $C_{(\mathcal{H}, j)}$ by

$$
C_{(\mathcal{H}, j)}(r)=C_{1}(r)+C_{2}(r)+C_{3}(r),
$$

we have finished the proof. The statement about $\ell_{\left(\mathcal{H}, j ; \eta_{\infty}\right)}$ follows immediately from (6.17) and (6.19) applied to the case of $\eta^{\prime}=\eta$ and $z^{\prime}=z^{+}(\eta), z=z^{-}(\eta)$. Note that in the latter case, the term $E\left(\mathcal{H}_{\eta \eta^{\prime}}\right)$ drops out.

Now we are ready to state the main theorem of this section, which concerns a general structure of Novikov Floer cycles over a Floer homotopy $(\mathcal{H}, j)$. This theorem in particular says that for a Floer homotopy $(\mathcal{H}, j)$, if there is a Floer trajectory issued at a peak of the given Floer cycle, all such trajectories must be 'long' uniformly over the interval $[0,1]$ in that its energy has uniform positive lower bound. We like to emphasize that such a property strongly depends on the cycle property of the chains.

This theorem is closely related to the well-known picture arising in the First Cancellation Theorem in the Morse theory [13]. More precisely, consider a oneparameter family of smooth functions $f_{s}$ such that $f_{s}, 0 \leq s<1$ are all Morse but $f_{1}$ contains a unique degenerate critical points $p$. Let $\left(p^{+}(s), p^{-}(s)\right)$ for $s \in[0,1]$ with $p^{+}(1)=p^{-}(1)$ be the continuous family of cancellation pair of critical points of Morse functions $f_{s}$ of Morse indices of $(k+1, k)$ that appear in the First Cancellation Theorem [13], the cancellation theorem implies that $p^{+}(s)$ cannot contribute to the (Morse) homology $H_{k+1}(M)$. Once one gets rid of the critical point $p^{+}(s)$, one can easily see that there is a constant $A>0$, independent of $s \in[1-\epsilon, 1)$ such that all the gradient trajectories issued at any critical point $p$ have length greater than $A$. In particular, the level of the boundary $\partial(p)$ is less than the level of $p$ at least by $A$.

Theorem 6.7. Let $\eta \in[0,1] \backslash(\mathcal{S i n g}(\mathcal{H}) \cup \mathcal{N} t(\mathcal{H}, j))$ be any Floer point. Then there exist constants $\delta_{1}(\mathcal{H})>0$ and $A_{(\mathcal{H}, j)}^{1}>0$ independent of $\eta$ and $[z, w]$, depending only on $(\mathcal{H}, j)$, such that the followings hold:

(1) if $[z, w] \in \operatorname{Crit} \mathcal{A}_{H(\eta)},\left[z^{\prime}, w^{\prime}\right] \in \partial_{(H(\eta), J(\eta)}([z, w])$, and

$$
\mathcal{A}_{H(\eta)}([z, w])-\mathcal{A}_{H(\eta)}\left(\left[z^{\prime}, w^{\prime}\right]\right)<A_{(\mathcal{H}, j)}^{1}
$$

then we have $d(\eta$, Sing $(\mathcal{H}))<\delta_{1}(\mathcal{H})$ and

$$
[z, w]=\left[z^{+}(\eta), w\right], \quad\left[z^{\prime}, w^{\prime}\right]=\left[z^{-}(\eta), w \# u^{c a n}\right] .
$$


(2) there exists another constant $\delta<\delta_{1}(\mathcal{H})$ such that if $d(\eta, \operatorname{Sing}(\mathcal{H}))<\delta$, we can write $\partial_{\left(J\left(\eta_{i}\right), H\left(\eta_{i}\right)\right)}\left(\left[z^{+}\left(\eta_{i}\right), w_{i}^{+}\right]\right)$as

$$
\partial_{\left(J\left(\eta_{i}\right), H\left(\eta_{i}\right)\right)}\left(\left[z^{+}\left(\eta_{i}\right), w_{i}^{+}\right]\right)=\left[z^{-}\left(\eta_{i}\right), w_{i}^{+} \# u_{i}\right]+\beta_{i}
$$

for a chain $\beta$ satisfying

$$
\lambda_{H(\eta)}(\beta) \leq \mathcal{A}_{H(\eta)}\left(\left[z^{-}(\eta), w \# u^{c a n}\right]-A_{(\mathcal{H}, j)}^{1} .\right.
$$

(3) Furthermore the constant $\min \left\{A_{(\mathcal{H}, j)}^{1}, A(\omega ; j)\right\}$ can be chosen to be lower semi-continuous in $j$.

Proof. We first note that

$$
\mathcal{A}_{H(\eta)}([z, w])>\lambda_{H(\eta)}\left(\partial_{(J(\eta), H(\eta))}([z, w])\right)
$$

in general as the Floer boundary map always decreases the level. Furthermore if $d(\eta, \operatorname{Sing}(\mathcal{H})) \geq \delta_{1}$, where $d(\eta, \mathcal{S i n g}(\mathcal{H}))$ is the distance of $\eta$ to $\operatorname{Sing}(\mathcal{H})$, Lemma A.3 (or rather its proof) implies that there exists $A=A\left(\mathcal{H}, j, \delta_{1}\right)>0$ such that

$$
\mathcal{A}_{H(\eta)}([z, w])-\mathcal{A}_{H(\eta)}\left(\left[z^{\prime}, w^{\prime}\right]\right) \geq A
$$

for any $[z, w] \in \operatorname{Crit} \mathcal{A}_{H(\eta)}$ and $\left[z^{\prime}, w^{\prime}\right] \in \partial([z, w])$.

Therefore from now on, we assume

$$
d(\eta, \mathcal{S i n g}(\mathcal{H}))<\delta_{1}
$$

and so the associated bifurcation branches $\left(z^{+}(\eta), z^{-}(\eta)\right)$ issued at one of the points in $\operatorname{Sing}(\mathcal{H})$ are defined. We define

$$
\begin{aligned}
A_{(\mathcal{H}, j)}^{1}:=\inf _{\eta, u}\left\{E_{(H(\eta), J(\eta))}(u) \mid \eta \in\right. & {[0,1] \backslash(\operatorname{Sing}(\mathcal{H}) \cup \mathcal{N} t(\mathcal{H}, j)), } \\
0 & <d(\eta, \operatorname{Sing}(\mathcal{H}))<\delta_{1}(\mathcal{H}), \\
u & \left.\notin \mathcal{M}\left(\mathcal{H}, j ; \rho ; z^{+}(\eta), z^{-}(\eta) ;\left[u^{c a n}\right]\right)\right\} .
\end{aligned}
$$

By definition, $A_{(\mathcal{H}, j)}^{1}$ satisfies (6.20). The rest of the proof will show that $A_{(\mathcal{H}, j)}^{1}>0$ and $A_{(\mathcal{H}, j)}^{1}$ is lower semi-continuous in $(\mathcal{H}, j)$.

Suppose that there exists a sequence $\eta_{i} \in[0,1] \backslash \mathcal{S i n g}(\mathcal{H}) \cup \mathcal{N} t(\mathcal{H}, j)$ with $\eta_{i} \rightarrow \eta_{\infty} \in(0,1)$ such that there exist critical points $\left[z_{i}, w_{i}\right] \in \operatorname{Crit} \mathcal{A}_{H\left(\eta_{i}\right)}$ and $\left[z_{i}^{\prime}, w_{i}^{\prime}\right] \in \partial_{\left(J\left(\eta_{i}\right), H\left(\eta_{i}\right)\right)}\left(\left[z_{i}, w_{i}\right]\right)$ satisfying

$$
\mathcal{A}_{H\left(\eta_{i}\right)}\left(\left[z_{i}, w_{i}\right]\right)-\mathcal{A}_{H\left(\eta_{i}\right)}\left(\left[z_{i}^{\prime}, w_{i}^{\prime}\right]\right) \rightarrow 0 .
$$

It will be enough to prove that (6.21) must hold under the given assumption, if $i$ is sufficiently large.

The rest of the proof will be divided into 5 steps.

Step 1: $\eta_{\infty} \in \operatorname{Sing}(\mathcal{H})$.

Suppose to the contrary that $\eta_{\infty} \in[0,1] \backslash \mathcal{S}$ ing $(\mathcal{H})$. Then the lower semicontinuity of $A_{(J, H)}$ stated in Proposition 6.2 implies

$$
A_{(H(\eta), J(\eta))} \geq \frac{1}{2} A_{\left(H\left(\eta_{\infty}\right), J\left(\eta_{\infty}\right)\right)}>0
$$


for all $\eta$ sufficiently close to $\eta_{\infty}$. In turn this implies

$$
\mathcal{A}_{H\left(\eta_{i}\right)}\left(\left[z_{i}, w_{i}\right]\right)-\mathcal{A}_{H\left(\eta_{i}\right)}\left(\left[z_{i}^{\prime}, w_{i}^{\prime}\right]\right)=E_{J\left(\eta_{i}\right)}(u) \geq \frac{1}{2} A_{\left(J\left(\eta_{\infty}\right), H\left(\eta_{\infty}\right)\right)}
$$

for any pair $\left[z_{i}, w_{i}\right] \neq\left[z_{i}^{\prime}, w_{i}^{\prime}\right]$ for all sufficiently large. This contradicts (6.23).

Step 2.

By the definition of the Cerf homotopy $\mathcal{H}$, there is exactly one birth-death or death-birth point at each $\eta_{\infty} \in \operatorname{Sing}(\mathcal{H})$. Then we can repeat the proof of Proposition 6.2 with minor modifications, and prove that except the critical points of the form $\left[z^{+}(\eta), w\right]$, where $z^{+}(\eta)$ is the upper one of the bifurcation pair $\left(z^{+}(\eta), z^{-}(\eta)\right)$ issued at $\eta_{\infty}$ as stated in Proposition 3.3, the levels of all other critical points are dropped down by a positive amount, say $B>0$, under the action by the boundary map $\partial_{(J(\eta), H(\eta))}$, i.e.,

$$
\lambda_{H\left(\eta_{i}\right)}\left(\partial_{\left(J\left(\eta_{i}\right), H\left(\eta_{i}\right)\right)}\left(\left[z_{i}, w_{i}\right]\right)\right) \leq \mathcal{A}_{H\left(\eta_{i}\right)}\left(\left[z_{i}, w_{i}\right]\right)-B .
$$

And $B$ can be chosen to be independent of any given Floer point $\eta$. This proves that $\left[z_{i}, w_{i}\right]$ must be of the form $\left[z^{+}\left(\eta_{i}\right), w_{i}\right]$.

Step 3: Finish-up of the proof of (1).

We now analyze the term $\partial_{H\left(\eta_{i}\right)}\left(\left[z^{+}\left(\eta_{i}\right), w_{i}\right]\right)$. First, we claim that we have

$$
\mathcal{A}_{H\left(\eta_{i}\right)}\left(\left[z^{+}\left(\eta_{i}\right), w_{i}\right]\right)-\mathcal{A}_{H\left(\eta_{i}\right)}([z, w]) \geq B
$$

for all the generators $[z, w] \in \partial_{H\left(\eta_{i}\right)}\left(\left[z^{+}\left(\eta_{i}\right), w_{i}\right]\right)$, except possibly

$$
\left[z^{-}, w_{i}^{+} \# u_{z^{+} z^{-}}^{c a n}\right]
$$

if we choose $B$ smaller if necessary. We now make this statement more precise.

Let $(\mathcal{H}, j)$ be a Floer homotopy in the sense of Definition 6.4 , and $\eta \in[0,1]$ be a Floer point. We denote by $\alpha$ a Floer cycle associated to $(J(\eta), H(\eta))$. Write

$$
\operatorname{Sing}(\mathcal{H})=\left\{s_{1}, \ldots, s_{k_{1}}\right\} .
$$

When $d(\eta, \operatorname{Sing}(\mathcal{H}))$ is sufficiently small, let $\left(z^{+}(\eta), z^{-}(\eta)\right)$ be the bifurcation pair issued from a point $s_{i} \in \mathcal{S} i n g(\mathcal{H})$. We will prove the following lemma in the Appendix, which will finish the proof of (1).

Lemma 6.8. Suppose $(\mathcal{H}, j)$ and $\eta$ are as above. Let $\left[z^{+}(\eta), w\right]$ be the generator of $\alpha$ having the highest level among the generators of the form $\left[z^{+}(\eta), w\right]$. Then there exists a constant $B=B(\mathcal{H}, j)>0$ such that there exists $\delta=\delta(\mathcal{H}, j, \epsilon)$ with

$$
0<\delta<\min _{1 \leq i \leq k_{1}-1}\left\{\left|s_{i+1}-s_{i}\right|\right\}
$$

such that for any $\eta$ with $\operatorname{dist}(\eta, \mathcal{S i n g}(\mathcal{H}))<\delta$, any element $u$ in

$$
\mathcal{M}\left(J(\eta), H(\eta) ;\left[z^{+}(\eta), w^{+}\right],[z, w]\right)
$$

satisfies

$$
E_{(J(\eta), H(\eta))}(u) \geq B
$$


unless $[z, w]=\left[z^{-}, w_{i} \# u^{c a n}\right]$.

Step 4: Proof of (6.22). To prove (2), we first study the moduli space

$$
\mathcal{M}\left(J\left(\eta_{i}\right), H\left(\eta_{i}\right) ;\left[z^{+}\left(\eta_{i}\right), w_{i}\right],\left[z^{-}\left(\eta_{i}\right), w_{i} \# u^{c a n}\right]\right)
$$

of solutions $u$ of (6.1) in the class prescribed by the condition

$$
u(-\infty)=z^{+}, u(\infty)=z^{-}, \quad w \# u^{c a n} \sim w \# u .
$$

We first note that the estimate for $\ell_{\left(\mathcal{H}, j ; \eta_{\infty}\right)}$ in Proposition 6.6 implies that all the elements in this moduli space has small energy and so is 'localized' near the unique degenerate periodic orbit $z_{\infty}$ of $H\left(\eta_{\infty}\right)$. In other words, the image of all the elements $u$ in $\mathcal{M}\left(J\left(\eta_{i}\right), H\left(\eta_{i}\right) ;\left[z^{+}\left(\eta_{i}\right), w^{+}\right],\left[z^{-}\left(\eta_{i}\right), w^{+} \# u^{\text {can }}\right]\right)$ is contained in a small neighborhood of that of $z_{\infty}$, which can be chosen as small as we want by choosing a sufficiently large $i$.

Then by considering the standard bifurcation picture near a generic degenerate periodic orbit with respect to a suitable family $J=\left\{J_{t}\right\}_{0<t<1}$ of almost complex structures (see $[6,11]$ for some relevant explanations), there exists such a family for which we have precisely one Floer trajectory $u_{i}$ from $z^{+}\left(\eta_{i}\right)$ to $z^{-}\left(\eta_{i}\right)$ that is homotopic to the canonical thin cylinder. We refer to [11, Theorem 9.9] for the precise statement and its proof. We like to remark that proving such a statement directly involves highly technical analytical estimates because it involves an analysis of the Floer moduli space near a degenerate Hamiltonian $H\left(\eta_{\infty}\right)$. The proof was outlined in [5] by Floer himself and later completed by Yi-Jen Lee [11].

Once we have this analytical theorem at our disposal, applying the standard cobordism argument over a homotopy from the above mentioned $J$ to our $J\left(\eta_{i}\right)$, we have proven that the matrix coefficient becomes

$$
\left\langle\partial_{\left(J\left(\eta_{i}\right), H\left(\eta_{i}\right)\right)}\left(\left[z^{+}\left(\eta_{i}\right), w_{i}\right]\right),\left[z^{-}\left(\eta_{i}\right), w_{i} \# u^{c a n}\right]\right\rangle=1 .
$$

(Here we remind the readers that in this paper we are assuming that $(M, \omega)$ is strongly semi-positive and so all the matrix coefficients of the basic operators in the Floer homology have integer coefficients. For the general $(M, \omega)$, this matrix coefficient may become rational numbers but will not still be zero.) Therefore we have

$$
\partial_{\left(J\left(\eta_{i}\right), H\left(\eta_{i}\right)\right)}\left(\left[z^{+}\left(\eta_{i}\right), w_{i}\right]\right)=\left[z^{-}\left(\eta_{i}\right), w_{i} \# u^{c a n}\right]+\beta_{i},
$$

where $\beta_{i}$ does not have $\left[z^{-}\left(\eta_{i}\right), w_{i} \# u^{c a n}\right]$ as one of its generators.

Step 5: Finish-up of the proof of (2).

Now we estimate the level of $\beta_{i}$. But Lemma 6.8 implies

$$
\mathcal{A}_{H\left(\eta_{i}\right)}\left(\left[z^{+}\left(\eta_{i}\right), w_{i}\right]\right)-\mathcal{A}_{H\left(\eta_{i}\right)}([z, w]) \geq B
$$

for any generator $[z, w] \in \beta_{i}$, where $B=B(\mathcal{H}, j)$. Combined with Step 4, this finishes the proof of (2).

Step 6: The lower semi-continuity of $A_{(\mathcal{H}, j)}^{1}$. 
The proof of the lower semi-continuity of $\min \left\{A_{(\mathcal{H}, j)}^{1}, A(\omega ; j)\right\}$ in $j$ can be proceeded as the proof of proof of Proposition 6.2 and so omitted. This finally finishes the proof of Theorem 6.7.

Theorem 6.7 gives rise to the following proposition.

Proposition 6.9. Let $a \neq 0$ be a given quantum cohomology class and denote by $\alpha$ a Floer cycle with $[\alpha]=a^{b}$. Then there exists $0<\delta_{3} \leq \delta_{1}$ with $\delta_{3}=$ $\delta_{3}(\mathcal{H}, j)$ such that for any Floer point $\eta$ satisfying $d(\eta, \mathcal{S i n g}(\mathcal{H}))<\delta_{3}$ for any tight Floer cycle $\alpha$ of $(H(\eta), J(\eta))$, any of its peaks cannot have the form

$$
\left[z^{+}(\eta), w\right]
$$

for any bounding disc $w$.

Proof. Suppose to the contrary that there exists a sequence of the Floer points $\eta_{k} \rightarrow \eta_{\infty} \in \mathcal{S i n g}(\mathcal{H})$ such that there exists a sequence of tight Floer cycles $\alpha_{k}$ whose peaks have the form $\left[z^{+}\left(\eta_{k}\right), w_{k}\right]$. By the definition of Cerf homotopies, there exists at most one such peak of the form $\left[z^{+}\left(\eta_{k}\right), w_{k}\right]$ provided $k$ is sufficiently large. We first derive

$$
\lambda_{H\left(\eta_{k}\right)}\left(\partial_{\left(H\left(\eta_{k}\right), J\left(\eta_{k}\right)\right)}\left(\left[z^{+}\left(\eta_{k}\right), w_{k}\right]\right)\right)=\mathcal{A}_{H\left(\eta_{k}\right)}\left(\left[z^{-}\left(\eta_{k}\right), w_{k} \# u^{c a n}\right]\right)
$$

from (6.25). Since $\left[z^{+}\left(\eta_{k}\right), w_{k}\right]$ is a peak of $\alpha_{k}$, we have

$$
\lambda_{H\left(\eta_{k}\right)}\left(\alpha_{k}\right)=\mathcal{A}_{H\left(\eta_{k}\right)}\left(\left[z^{+}\left(\eta_{k}\right), w_{k}\right]\right)
$$

and Proposition 6.5 implies

$$
\begin{aligned}
\mathcal{A}_{H\left(\eta_{k}\right)}\left(\left[z^{-}\left(\eta_{k}\right), w_{k} \# u^{c a n}\right]\right) & \geq \lambda_{H\left(\eta_{k}\right)}\left(\alpha_{k}\right)-\ell\left(\mathcal{H}, j ; \eta_{0}\right)\left(\left|\eta_{k}-\eta_{0}\right|\right) \\
& =\rho\left(H\left(\eta_{k}\right) ; a\right)-\ell\left(\mathcal{H}, j ; \eta_{0}\right)\left(\left|\eta_{k}-\eta_{0}\right|\right) .
\end{aligned}
$$

From the proof of Theorem 6.7 or more specifically from (6.24), we have

$$
\lambda_{H\left(\eta_{k}\right)}\left(\partial_{\left(H\left(\eta_{k}\right), J\left(\eta_{k}\right)\right)}([z, w])\right) \leq \rho\left(H\left(\eta_{k}\right) ; a\right)-B
$$

if $z \neq z^{+}\left(\eta_{k}\right)$, where $B$ is the same constant used in the proof of Theorem 6.7. On the other hand if $z=z^{+}(\eta)$, then obviously we have

$$
\begin{aligned}
& \lambda_{H\left(\eta_{k}\right)}\left(\partial_{\left(H\left(\eta_{k}\right), J\left(\eta_{k}\right)\right)}\left(\left[z^{+}\left(\eta_{k}\right), w_{k}\right]\right)\right) \\
= & \mathcal{A}_{H\left(\eta_{k}\right)}\left(\left[z^{-}\left(\eta_{k}\right), w_{k} \# u^{c a n}\right]\right)>\mathcal{A}_{H\left(\eta_{k}\right)}\left(\left[z^{-}\left(\eta_{k}\right), w \# u^{c a n}\right]\right) \\
= & \lambda_{H\left(\eta_{k}\right)}\left(\partial_{\left(H\left(\eta_{k}\right), J\left(\eta_{k}\right)\right)}\left(\left[z^{+}\left(\eta_{k}\right), w\right]\right)\right)
\end{aligned}
$$

for any other $\left[z^{+}\left(\eta_{k}\right), w\right] \neq\left[z^{+}\left(\eta_{k}\right), w_{k}\right]$. Therefore if we write $\partial_{\left(H\left(\eta_{k}\right), J\left(\eta_{k}\right)\right)}\left(\alpha_{k}\right)=\partial_{\left(H\left(\eta_{k}\right), J\left(\eta_{k}\right)\right)}\left(\left[z^{+}\left(\eta_{k}\right), w_{k}\right]\right)+\partial_{\left(H\left(\eta_{k}\right), J\left(\eta_{k}\right)\right)}\left(\alpha_{i}-\left[z^{+}\left(\eta_{k}\right), w_{k}\right]\right)$ (6.29) and (6.30) imply

$$
\lambda_{H\left(\eta_{k}\right)}\left(\partial_{\left(H\left(\eta_{k}\right), J\left(\eta_{k}\right)\right)}\left(\alpha_{i}-\left[z^{+}\left(\eta_{k}\right), w_{k}\right]\right)\right)<\lambda_{H\left(\eta_{k}\right)}\left(\partial_{\left(H\left(\eta_{k}\right), J\left(\eta_{k}\right)\right)}\left(\left[z^{+}\left(\eta_{k}\right), w_{k}\right]\right)\right) .
$$


In particular, $\partial_{\left(H\left(\eta_{k}\right), J\left(\eta_{k}\right)\right.}\left(\alpha_{k}\right)$ cannot vanish which contradicts that $\alpha_{k}$ is a cycle. Hence the proof.

\section{§7. Handle sliding lemma and sub-homotopies}

In this section, we recall another important element in the chain level theory, the handle sliding lemma introduced in [15]. We state the most natural version of the handle sliding lemma which uses the sub-homotopies of the given homotopy $\mathcal{H}$ instead of the piecewise-linear approximation of $\mathcal{H}$ which was used in $[15]$.

We start with the following lemma from [15]. Partly for the reader's convenience and also because we need to add some important points to the lemma, we repeat its proof here.

Lemma 7.1 ([15, Lemma 5.1]). Let $(\mathcal{H}, j)$ be one-parameter family such that $\left.\mathcal{H} \in \mathcal{P}^{\text {reg }}\left(\mathcal{H}_{m}(M) ; H_{1}, H_{2}\right)\right)$. For each $\eta \in[0,1] \backslash \operatorname{Sing}(\mathcal{H})$, we define

$$
\begin{aligned}
A_{(H(\eta), J(\eta))}^{0}=\inf _{u}\left\{\int\left|\frac{\partial u}{\partial \tau}\right|_{J(\eta)}^{2} \mid \text { u satisfies }(6.1),\right. & \\
& \text { is not stationary and Index } u=0\}
\end{aligned}
$$

and

$$
A_{(\mathcal{H}, j)}^{r e g, 0}=\inf _{s \in[0,1] \backslash \mathcal{S i n g}(\mathcal{H})} A_{\left(H^{s}, J^{s}\right)}^{0}
$$

Then $A_{(\mathcal{H}, j)}^{\text {reg,0 }}$ is strictly positive.

Proof. Suppose the contrary that $A_{(\mathcal{H}, j)}^{r e g, 0}=0$, i.e., that there exists a sequence $\eta_{k} \in[0,1] \backslash \operatorname{Sing}(\mathcal{H})$ with $\eta_{k} \rightarrow \eta_{\infty} \in(0,1)$ and $u_{k}$ solutions of $(6.1)$ for $\left(H\left(\eta_{k}\right), J\left(\eta_{k}\right)\right)$ such that

$$
\int\left|\frac{\partial u_{k}}{\partial \tau}\right|_{J\left(\eta_{k}\right)}^{2} \rightarrow 0, \quad \text { Index } u_{k}=0
$$

Then we must have, by choosing a subsequence if necessary,

$$
\eta_{\infty} \in \operatorname{Sing}(\mathcal{H})
$$

and a degenerate periodic orbit $z_{\infty}$ of $\dot{x}=X_{H\left(\eta_{\infty}\right)}(x)$ such that $u_{k} \rightarrow z_{\infty}$ uniformly and so

$$
u_{k}(\infty), u_{k}(-\infty) \rightarrow z_{\infty}
$$

Since $u_{k}( \pm \infty)$ are solutions of $\dot{x}=X_{H\left(\eta_{k}\right)}(x)$, they must be the pair described in (1) right above (3.1) in Section 3.1 and hence

$$
\operatorname{Index}\left(u_{k}\right)=\mu\left(\left[z^{+}\left(\eta_{k}\right), w_{k}^{+}\right]\right)-\mu\left(\left[z^{-}\left(\eta_{k}\right), w_{k}^{-}\right]=1\right. \text {. }
$$

But this contradicts the index condition in the definition of $A_{\left(H^{s}, J^{s}\right)}^{0}$ which finishes the proof. 
Next we define

$$
A_{(\mathcal{H}, j)}^{\text {sing }}=\min _{k}\left\{A_{\left(H^{s_{k}, J^{s}}\right)} \mid s_{k} \in \mathcal{S i n g}(\mathcal{H})\right\} .
$$

This is again positive by a Gromov type compactness theorem. Now we have the following crucial definition of a family version of the constant $A_{(H, J)}$ suitable for our purpose. We define

$$
A_{(\mathcal{H}, j)}^{0}=\min \left\{A_{(\mathcal{H}, j)}^{\text {reg, }}, A_{(\mathcal{H}, j)}^{\text {sing }}, A(\omega ; j)\right\}
$$

which we know is strictly positive.

Remark 7.2. We would like to point out that all the invariants $A_{(\mathcal{H}, j)}^{\text {sing }}, A_{(\mathcal{H}, j)}^{\text {reg,0 }}$ and $A_{(\mathcal{H}, j)}^{0}$ are defined in terms of the Floer boundary equation (4.3), not in terms of the continuity equation (4.5). Furthermore, it follows from the same kind of proof as the proofs of Proposition 6.1 and 6.2 that for a fixed $\mathcal{H}$,

$$
\min \left\{A_{(\mathcal{H}, j)}^{0}, A(\omega ; j)\right\}
$$

is lower semi-continuous in $j$.

We state the following simple lemma, or rather an observation from this remark, which follows immediately from the definitions of $A_{(\mathcal{H}, j)}^{0}$ and of the sub-homotopy in Definition 3.8. This turns out to play an important role in our proof of the main theorem later, and is one of the reasons why we have to use the sub-homotopies of the given homotopy.

Lemma 7.3. Let $(\mathcal{H}, j)$ be a given homotopy. Then we have the inequality

$$
A_{\left(\mathcal{H}_{\eta_{1} \eta_{2}}, j_{\eta_{1} \eta_{2}}\right)}^{0} \geq A_{(\mathcal{H}, j)}^{0}
$$

for the sub-homotopy $\mathcal{H}_{\eta_{1} \eta_{2}}$ between any two Floer points $0 \leq \eta_{1} \leq \eta_{2} \leq 1$. If $\eta_{1}>\eta_{2}$ instead, then we have

$$
A_{\left(\mathcal{H}_{\eta_{1} \eta_{2}}^{-1}, j_{\eta_{1} \eta_{2}}^{-1}\right)}^{0} \geq A_{\left(\mathcal{H}^{-1}, j^{-1}\right)}^{0} .
$$

Proof. The proof is an immediate consequence of the definitions of $A_{(\mathcal{H}, j)}^{0}$ and of the sub-homotopy in general.

We now recall the following handle sliding lemma from [15]. We, however, add an important improvement from that of [15]: here we used the subhomotopy (4.7) of $\mathcal{H}$ instead of the linear homotopy that was used in [15]. It turns out that this usage of sub-homotopies is the most natural and the optimal choice, in that the constant associated to the given $(\mathcal{H}, j)$ in Definition 5.4 can be used for all its sub-homotopies. The proof here is taken from [15].

Proposition 7.4 (The handle sliding lemma). Let $j=\left\{J^{\eta}\right\} \in \mathcal{P}^{\operatorname{Cerf}}\left(j_{\omega} ; \mathcal{H}\right)$ be a (two parameter) family of almost complex structures and $\{H(\eta)\}_{0 \leq \eta \leq 1}$ be a generic family of Hamiltonians. Fix a cut-off function $\rho$. Let $A_{(\mathcal{H}, j)}^{0}$ be the constant defined in (7.4) and let $\eta_{1}, \eta_{2} \in[0,1]$. 
(1) Then there exists $\delta=\delta(\mathcal{H}, j)>0$ such that if $\left|\eta_{1}-\eta_{2}\right|<\delta$, any finite energy solution $u$ with

$$
\text { Index } u=0
$$

of (5.3) must either satisfy

$$
\int\left|\frac{\partial u}{\partial \tau}\right|_{J \rho(\tau)}^{2} \leq \varepsilon(\delta)
$$

or

$$
\int\left|\frac{\partial u}{\partial \tau}\right|_{J^{\rho(\tau)}}^{2} \geq A_{(\mathcal{H}, j)}^{0}-\varepsilon(\delta),
$$

where for $\varepsilon(\delta) \rightarrow 0$ as $0<\delta \rightarrow 0$, provided $\delta \leq \delta_{0}$.

(2) In addition, in the case of (7.6), $u$ is homotopic to the canonical cylin-

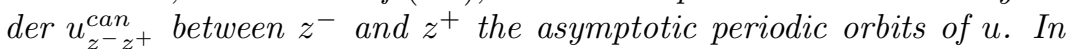
particular, we have

$$
\int\left|\frac{\partial u}{\partial \tau}\right|_{J \rho(\tau)}^{2} \rightarrow 0
$$

as $\delta \rightarrow 0$.

(3) Furthermore the same constant $A_{(\mathcal{H}, j)}^{0}$ for $(7.7)$ can be used for all the sub-homotopies $\mathcal{H}_{\eta_{1} \eta_{2}}$ for any two Floer points $\eta_{1}, \eta_{2}$.

Proof. We prove this by contradiction. Suppose the contrary that there exists some $\varepsilon>0, \eta_{1}$ and $\eta_{i}$ with $\eta_{i} \rightarrow \eta_{1}$ as $i \rightarrow \infty$, and solutions $u_{i}$ that satisfy

Index $u_{i}=0$,

$$
\frac{\partial u_{i}}{\partial \tau}+J^{\rho(\tau)}\left(\frac{\partial u_{i}}{\partial t}-X_{H^{\rho(\tau)}}\left(u_{i}\right)\right)=0
$$

and

$$
\varepsilon<\int\left|\frac{\partial u_{i}}{\partial \tau}\right|_{J^{\rho(\tau)}}^{2}<A_{(\mathcal{H}, j)}^{0}-\varepsilon .
$$

In particular, the right half of (7.9) implies the uniform bound on the energy of $u_{i}$. As $i \rightarrow \infty$, the equation (7.8) converges to (6.1) with $(H, J)=$ $\left(H\left(\eta_{1}\right), J\left(\eta_{1}\right)\right)$. By Gromov's type compactness theorem, we have a cusp trajectory

$$
u_{\infty}=\sum_{k} u_{\infty, k}
$$

which is the limit of a subsequence, where each $u_{\infty, k}$ is a solution of (5.1) for $H=H\left(\eta_{1}\right)$, possibly with a finite number of bubbles attached. We also have

$$
\lim _{i} E_{\left(H^{\rho(\tau)}, J^{\rho(\tau)}\right)}\left(u_{i}\right)=\sum_{k} E_{\left(H^{\rho(\tau)}, J^{\rho(\tau)}\right)}\left(u_{\infty, k}\right)
$$

On the other hand the left half of (7.9) implies that at least one of $u_{\infty, k}$ is not trivial, i.e., not stationary. 
Now we consider three cases separately: the first is the one where $\eta_{1} \in$ $\operatorname{Sing}(\mathcal{H})$ and the second where $\eta_{1} \in \mathcal{N} t(\mathcal{H}, j)$ and the rest where

$$
\eta_{1} \in[0,1] \backslash(\operatorname{Sing}(\mathcal{H}) \cup \mathcal{N} t(\mathcal{H}, j)) .
$$

When $\eta_{1} \in \mathcal{S}$ ing, we must have

$$
\lim _{i} E_{\left(H^{\rho(\tau)}, J^{\rho(\tau)}\right)}\left(u_{i}\right) \geq \min \left\{A_{\left(H\left(\eta_{1}\right), J\left(\eta_{1}\right)\right)}^{\operatorname{sing}}, A(\omega ; j)\right\} \geq A_{(\mathcal{H}, j)}^{0}
$$

which gives rise to a contradiction to (7.9) when $i$ is sufficiently large. On the other hand, if $\eta_{1} \in \mathcal{N} t(\mathcal{H}, j)$, the cusp curve must contain a component $u_{\infty}$ that has Index 0 and is non-constant. Again the right hand side inequality of (7.9) prevents this from happening. Finally when $\eta_{1} \in[0,1] \backslash(\mathcal{S i n g}(\mathcal{H}) \cup \mathcal{N} t(\mathcal{H}, j))$, the index condition Index $u_{i}=0$ and the transversality condition imply that all the components $u_{\infty, k}$ must be constant which again contradicts to the left hand side inequality of (7.9) if $i$ is sufficiently large. This finishes the proof of the handle sliding lemma.

\section{$\S 8$. Parametric stability of tightness of Novikov Floer cycles}

This is the key section which will involve all the results we proved in Section 3-7, especially Theorem 3.7, Theorem 4.6, Proposition 5.7, Theorem 6.7 and Proposition 7.4.

Let

$$
a=\sum a_{A} q^{-A}, \quad a_{A} \in H^{*}(M)
$$

be a non-zero quantum cohomology class. We denote by $\Gamma(a) \subset \Gamma$ the set of $A$ 's for which the coefficient $a_{A}$ is non-zero. By the definition of the Novikov ring, we can enumerate $\Gamma(a)$ so that

$$
-\lambda_{1}<-\lambda_{2}<\cdots<-\lambda_{j}<\cdots,
$$

where $\lambda_{j}=\omega\left(A_{j}\right)$. We call the first term $a_{1} q^{-A_{1}}$ the leading order term of the quantum cohomology class $a$ and denote by $\operatorname{Ld}(a)$.

We recall from $[18]$ that for a given quantum cohomology class $a \neq 0$, we define the mini-max value of $\mathcal{A}_{H}$

$$
\rho(H ; a)=\inf _{\alpha}\left\{\lambda_{H}(\alpha) \mid \alpha \in \operatorname{ker} \partial, \text { with }[\alpha]=a^{b}\right\}
$$

for a nondegenerate Hamiltonian $H$ for which the Floer homology $H F_{*}(H, J)$ is defined for a generic choice of $J$. As we pointed out in [18], the number $\rho(H ; a)$ is independent of the choice of $J$.

The following notion of tight Floer cycles introduced in [18] is important in the proof of Theorem II.

Definition 8.1. Let $(H, J)$ be a Floer regular pair so that the Floer complex $\left(C F_{*}(H), \partial_{(H, J)}\right)$ is defined. Let $\alpha$ be a Floer cycle of $H$ and $a \in Q H^{*}(M)$ be 
the corresponding quantum cohomology class with $[\alpha]=a^{b}$. We call the Floer cycle $\alpha$ of $(H, J)$ tight if it realizes the mini-max value, i.e.,

$$
\lambda_{H}(\alpha)=\rho(H ; a) .
$$

We call a critical value $\lambda$ of $\mathcal{A}_{H}$ a homologically essential critical value of $\mathcal{A}_{H}$, if there exists $J$ such that $(H, J)$ is Floer regular, and $\lambda=\lambda_{H}(\alpha)$ for a tight Floer cycle of $(H, J)$.

Although the homologically essentialness of a critical point $[z, w]$ of $\mathcal{A}_{H}$ depends on the choice of $J$, the following proposition proves that the homologically essentialness of a critical value is independent of the choice of $H$-regular $J$ 's.

Proposition 8.2. Let $J, J^{\prime}$ be two $H$-regular one-periodic family of almost complex structures. Suppose that a Floer cycle $\alpha$ of $(H, J)$ is tight and assume that $j$ is a homotopy connecting $J, J^{\prime}$ that is $\mathcal{H}$-regular for the constant homotopy $\mathcal{H} \equiv H$. Then the transferred cycle

$$
h_{(\mathcal{H}, j ; \rho)}(\alpha)
$$

defines a tight Floer cycle for the pair $\left(H, J^{\prime}\right)$.

Proof. Since $\left[h_{(H, j ; \rho)}(\alpha)\right]=a^{b}$, we have

$$
\lambda_{H}\left(h_{(H, j ; \rho)}(\alpha)\right) \geq \rho(H ; a)=\lambda_{H}(\alpha) .
$$

Next let $\left[z^{\prime}, w^{\prime}\right]$ be a peak of $h_{(H, j ; \rho)}(\alpha)$. By the definition of $h_{(H, j ; \rho)}(\alpha)$, there is $[z, w] \in \alpha$ such that $\mathcal{M}\left((H, j ; \rho) ;[z, w],\left[z^{\prime}, w^{\prime}\right]\right) \neq \emptyset$. Then (5.8) applied to the constant homotopy $\mathcal{H} \equiv H$ implies

$$
\mathcal{A}_{H}\left(\left[z^{\prime}, w^{\prime}\right]\right) \leq \mathcal{A}_{H}([z, w])
$$

which implies

$$
\lambda_{H}\left(h_{(H, j ; \rho)}(\alpha)\right) \leq \lambda_{H}(\alpha) .
$$

Combining (8.2) and (8.3), we have proved

$$
\lambda_{H}\left(h_{(H, j ; \rho)}(\alpha)\right)=\rho(H ; a)
$$

which finishes the proof.

Now we fix a homotopy $\mathcal{H} \in \mathcal{P}^{\operatorname{Cerf}}\left(\mathcal{H}_{m}(M) ; \epsilon f, H\right)$ and $j \in \mathcal{P}^{\text {tran }}\left(j_{\omega} ; \mathcal{H}\right)$, satisfying the properties described in Theorem 4.6,

$$
\mathcal{H}=\{H(\eta)\}_{0 \leq \eta \leq 1}, \quad j=\{J(\eta)\}_{0 \leq \eta \leq 1}
$$

such that

$$
H(0)=H_{\alpha}, \quad H(1)=H_{\beta}
$$

are nondegenerate. In particular, by the choice of $j$, the Floer homology $H F_{*}(H(\eta), J(\eta))$ is defined for any $\eta \in I(\mathcal{H}, j)$. 
Let $\eta_{0}$ be a Floer point at which there exists a tight Floer cycle $\alpha_{0} \in$ $C F_{*}\left(H\left(\eta_{0}\right)\right)$. We fix a homotopy

$$
j^{\eta_{0}} \in \mathcal{P}^{s u b}\left(j_{\omega} ; \mathcal{H} ; \eta_{0}\right) ; \quad j^{\eta_{0}}=\left\{J_{t}^{\eta_{0}}\right\}_{0 \leq t \leq 1}
$$

that satisfies the properties of Theorem 4.6 for the pair $\left(\mathcal{H}, j^{\eta_{0}}\right)$ and also satisfies

$$
J^{\eta_{0}}\left(\eta_{0}\right)=J\left(\eta_{0}\right)
$$

The main result of this section is to show that the tight Floer cycle $\alpha_{0}$ of $\left(H\left(\eta_{0}\right), J\left(\eta_{0}\right)\right)$, after perturbing $j$ slightly to $j^{\eta_{0}}$ as above, is parametrically stable in that there exists $\delta>0$ such that for any Floer point $\eta$ with $\left|\eta-\eta_{0}\right|<\delta$, $\rho(H(\eta) ; a)$ is the level of a tight Floer cycle of the pair $\left(H(\eta), J^{\eta_{0}}(\eta)\right)$. Note that once we know the latter fact, Proposition 8.2 implies that $\rho(H ; a)$ is indeed the level of a tight Floer cycle of $(H(\eta), J(\eta))$ for the original homotopy $(\mathcal{H}, j)$.

We fix a cut-off function $\rho_{0}: \mathbb{R} \rightarrow[0,1]$ and fix one $j^{\eta_{0}}$ as in (8.4) and satisfying (8.5). Then consider the corresponding chain map

$$
h_{\left(\eta_{0} \eta ; \rho_{0}\right)}:=h_{\left(\mathcal{H}_{\eta_{0} \eta}, j_{\eta_{0} \eta}^{\eta_{0}} ; \rho_{0}\right)}: C F_{*}\left(H\left(\eta_{0}\right)\right) \rightarrow C F_{*}(H(\eta)) .
$$

Theorem 8.3. Let $(\mathcal{H}, j)$ be as above and $0 \neq a \in Q H^{*}(M)$. Suppose that $\eta_{0} \in[0,1]$ is a Floer point at which a carries a tight Floer cycle for $H\left(\eta_{0}\right)$. Then there exists $\delta_{4}=\delta_{4}\left(\mathcal{H}, j^{\eta_{0}}, a ; \eta_{0}\right)>0$ such that on each of the semi-intervals $\left[\eta_{0}, \eta_{0}+\delta_{4}\right)$ or $\left(\eta_{0}-\delta_{4}, \eta_{0}\right]$ a carries tight cycles given by the transferred cycles $h_{\left(\eta_{0} \eta ; \rho_{0}\right)}\left(\alpha_{ \pm}\right)$of some tight cycles $\alpha_{ \pm}$at $\eta_{0}$ respectively.

We would like to remark that when $\eta_{0} \notin \mathcal{C}^{\text {nd }} \operatorname{ross}(\mathcal{H})$, we may take $\alpha_{-}=\alpha_{+}$. On the other hand, if $\eta_{0} \in \mathcal{C}^{n d} \operatorname{ross}(\mathcal{H})$, the two cycles $\alpha_{-}$and $\alpha_{+}$could be different.

The rest of the section will be occupied by the proof of this theorem.

We note that since $\eta_{0}$ is a Floer point, there are only a finite number of periodic orbits of $H\left(\eta_{0}\right)$, and can apply Theorem 6.7 for all Floer chains of $\left(H\left(\eta_{0}\right), J\left(\eta_{0}\right)\right)$.

By Theorem 4.6, the chain map (8.6) is defined at any point $\eta \in I\left(\mathcal{H}, j^{\eta_{0}} ; \eta_{0}\right)$, a dense subset of $[0,1]$. We emphasize that we need to choose $j^{\eta_{0}}$ depending on the point $\eta_{0}$ to ensure the properties stated in Theorem 4.6.

We now prove the following key proposition. We would like to emphasize that this kind of continuity statement in the levels of cycles, not in the levels of the corresponding homologies, does not hold in general, and even if it holds so, proving such a continuity statement is a highly non-trivial matter due to the phenomenon of cancellation of critical points. This is the reason why the structure theorem, Theorem 6.7, of Floer cycles proven in Section 6 is so crucial in our proof. 
Proposition 8.4. Let $(\mathcal{H}, j), \alpha_{0}, \eta_{0}$ and $j^{\eta_{0}}$ be as above. Then there exists some $\delta_{5}=\delta_{5}\left(\mathcal{H}, j^{\eta_{0}}, \eta_{0}\right)>0$ such that the assignment

$$
\eta \mapsto \lambda_{H(\eta)}\left(h_{\left(\eta_{0} \eta ; \rho_{0}\right)}\left(\alpha_{0}\right)\right)
$$

is continuous on $\left(\eta_{0}-\delta_{5}, \eta_{0}+\delta_{5}\right) \cap I\left(\mathcal{H}, j^{\eta_{0}} ; \eta_{0}\right)$.

Proof. Let $\delta>0$ which is to be determined later and consider the function

$$
\mu(\eta)=\lambda_{H(\eta)}\left(h_{\left(\eta_{0} \eta ; \rho_{0}\right)}\left(\alpha_{0}\right)\right) .
$$

We remind the readers that by definition all the generators of a Floer cycle representing the class dual to a given $a \in Q H^{*}(M)$ have the same ConleyZehnder indices.

For the proof of the proposition, we will follow the scheme used in the appendix of [15]. We first note that

$$
\mu(\eta)=\lambda_{H(\eta)}\left(h_{\left(\eta_{0} \eta ; \rho_{0}\right)}\left(\alpha_{0}\right)\right) \geq \rho(H(\eta) ; a)
$$

by the definition of $\rho(H(\eta) ; a)$ since we have $\left[h_{\eta_{0} \eta}\left(\alpha_{0}\right)\right]=a^{b}$. Hence $\mu(\eta)$ is finite and well-defined.

Once the finiteness of $\mu(\eta)$ is shown, the proposition will be an immediate consequence of the following lemma.

Lemma 8.5. There exists $\delta_{5}=\delta_{5}\left(\mathcal{H}, j^{\eta_{0}} ; \eta_{0}\right)>0$ for which we have the following inequality

$$
-E\left(\mathcal{H}_{\eta \eta^{\prime}}\right) \leq \mu\left(\eta^{\prime}\right)-\mu(\eta) \leq E\left(\mathcal{H}_{\eta \eta^{\prime}}\right)
$$

for any Floer points $\eta, \eta^{\prime} \in\left(\eta_{0}-\delta_{5}, \eta_{0}-\delta_{5}\right)$. In particular $\mu$ is continuous at $\eta_{0}$.

Therefore we will prove this lemma in the rest of the proof of Proposition 8.4. The proof of this lemma is quite long and intricate, and various arguments used in the proof touch the heart of the chain level Floer theory.

We first consider the case $\eta_{0}<\eta<\eta^{\prime}$ and provide complete details of the proof of (8.7) in this case. We will briefly mention the proof of (8.7) for the other cases in the end of the proof.

We compare the homotopy $\mathcal{H}_{\eta_{0} \eta^{\prime}}$ with the glued homotopy $\mathcal{H}_{\eta \eta^{\prime}} \# \mathcal{H}_{\eta_{0} \eta}$. We recall the general gluing identity from (5.24)

$$
h_{\mathcal{H}_{\eta_{0} \eta} \#(\rho ; R)} \mathcal{H}_{\eta \eta^{\prime}}=h_{\left(\eta \eta^{\prime} ; \rho_{1}\right)} \circ h_{\left(\eta_{0} \eta ; \rho_{0}\right)}
$$

for $\rho=\left(\rho_{0}, \rho_{1}\right)$ and for a sufficiently large $R>0$. We also assume $\rho_{1}$ is monotone. Then, if $\left[z_{\eta^{\prime}}^{\prime}, w_{\eta^{\prime}}^{\prime}\right]$ is a peak of the cycle $h_{\mathcal{H}_{\eta_{0} \eta} \#(\rho ; R)} \mathcal{H}_{\eta \eta^{\prime}}\left(\alpha_{0}\right)$, by the definition of the chain map $h_{\left(\eta \eta^{\prime} ; \rho_{1}\right)}$, there must exist some $\left[z^{\prime}, w^{\prime}\right] \in h_{\eta_{0} \eta}\left(\alpha_{0}\right)$ for which

$$
\mathcal{M}\left(\left(\mathcal{H}_{\eta \eta^{\prime}}, j_{\eta \eta^{\prime}} ; \rho_{1}\right) ;\left[z^{\prime}, w^{\prime}\right],\left[z_{\eta^{\prime}}^{\prime}, w_{\eta^{\prime}}^{\prime}\right]\right) \neq \emptyset
$$

holds. Now we state the following easy general lemma. 
Lemma 8.6. Let $F$ and $K$ be two nondegenerate Hamiltonians and $\mathcal{H}$ be a homotopy from $F$ to $K$ and $j$ be given such that $(\mathcal{H}, j)$ is Floer regular. Let $\rho$ be a given monotone cut-off function and let $h_{\mathcal{H}, j ; \rho}$ be the corresponding Floer homotopy map. Then we have

$$
\lambda_{K}\left(h_{(\mathcal{H}, j ; \rho)}(\alpha)\right) \leq \lambda_{F}(\alpha)+E^{-}(\mathcal{H})
$$

for any Floer chain $\alpha$ of $F$.

Proof. Let $\left[z^{\prime}, w^{\prime}\right]$ be a peak of the cycle $h_{(\mathcal{H}, j ; \rho)}(\alpha)$. By the definition of $h_{(\mathcal{H}, j ; \rho)}(\alpha)$, there is a generator $[z, w] \in \alpha$ such that

$$
\mathcal{M}\left((\mathcal{H}, j ; \rho) ;[z, w],\left[z^{\prime}, w^{\prime}\right]\right) \neq \emptyset
$$

Then (5.10) implies

$$
\mathcal{A}_{K}\left(\left[z^{\prime}, w^{\prime}\right]\right) \leq \mathcal{A}_{F}([z, w])+E^{-}\left(\mathcal{H}_{\eta \eta^{\prime}}\right)
$$

Since $\left[z^{\prime}, w^{\prime}\right]$ is a peak of the cycle $h_{(\mathcal{H} ; \rho)}(\alpha)$, i.e., $\mathcal{A}_{K}\left(\left[z^{\prime}, w^{\prime}\right]\right)=\lambda_{K}\left(h_{(\mathcal{H}, j ; \rho)}(\alpha)\right)$ and $\mathcal{A}_{F}([z, w]) \leq \lambda_{F}(\alpha)$, the lemma follows from (8.10).

Going back to the proof, Lemmas 5.5, 5.6, (8.8) and Lemma 8.6 imply

$$
\begin{aligned}
\lambda_{H\left(\eta^{\prime}\right)}\left(h_{\mathcal{H}_{\eta_{0} \eta} \#(\rho ; R)} \mathcal{H}_{\eta \eta^{\prime}}\left(\alpha_{0}\right)\right) & \leq \lambda_{H(\eta)}\left(h_{\left(\eta_{0} \eta ; \rho_{0}\right)}\left(\alpha_{0}\right)\right)+E^{-}\left(\mathcal{H}_{\eta \eta^{\prime}}\right) \\
& =\mu(\eta)+E^{-}\left(\mathcal{H}_{\eta \eta^{\prime}}\right) .
\end{aligned}
$$

We next deform the homotopy $\mathcal{H}_{\eta_{0} \eta} \#(\rho ; R) \mathcal{H}_{\eta^{\prime} \eta}$ to the $\rho_{0}$-elongated homotopy of $\mathcal{H}_{\eta_{0} \eta^{\prime}}$ for the given fixed choice of $\rho_{0}$ that we used in the definition of $\mu$ in the beginning. On the other hand, since we assume that $\eta_{0}<\eta<\eta^{\prime}$, $\mathcal{H}_{\eta_{0} \eta} \#{ }_{(\rho ; R)} \mathcal{H}_{\eta \eta^{\prime}}$ is also an elongation of the sub-homotopy $\mathcal{H}_{\eta_{0} \eta^{\prime}}$ (by a monotone cut-off function). More explicitly, if we denote by $\rho_{\text {elng }}$ the corresponding cut-off function, $\rho_{\text {elng }}$ is given by the formula (5.22).

Now we consider the homotopy

$$
\left(\bar{j}_{\eta_{0} \eta^{\prime}}, \overline{\mathcal{H}}_{\eta_{0} \eta^{\prime}} ; \bar{\rho}\right)
$$

between the $\rho_{0}$-elongation of $\left(\mathcal{H}, j^{\eta_{0}}\right)$ and the $\rho_{\text {elng }}$-elongation of $\left(\mathcal{H}, j^{\eta_{0}}\right)$ connected by the homotopy $\bar{\rho}=\{\rho(\kappa)\}_{0 \leq \kappa \leq 1}$ of cut-off functions

$$
\bar{\rho}=\{\rho(\kappa)\}_{0 \leq \kappa \leq 1} ; \quad \rho(0)=\rho_{0}, \rho(1)=\rho_{\text {elng }} .
$$

Since $\alpha_{0}$ is a cycle, we derive, from Proposition 5.7,

$$
h_{\left(\eta_{0} \eta^{\prime} ; \rho_{0}\right)}\left(\alpha_{0}\right)=h_{\mathcal{H}_{\eta_{0} \eta} \#(\rho ; R)} \mathcal{H}_{\eta \eta^{\prime}}\left(\alpha_{0}\right)+\partial_{\left(J\left(\eta^{\prime}\right), H\left(\eta^{\prime}\right)\right)} \circ H_{\left(\bar{j}_{\eta_{0} \eta^{\prime}}, \overline{\mathcal{H}}_{\eta_{0} \eta^{\prime}} ; \bar{\rho}\right)}\left(\alpha_{0}\right)
$$

with the inequality

$$
\lambda_{H\left(\eta^{\prime}\right)}\left(H_{\left(\bar{j}_{\eta_{0} \eta^{\prime}}, \overline{\mathcal{H}}_{\eta_{0} \eta^{\prime}} ; \bar{\rho}\right)}\left(\alpha_{0}\right)\right) \leq \lambda_{H\left(\eta_{0}\right)}\left(\alpha_{0}\right)+E\left(\mathcal{H}_{\eta_{0} \eta^{\prime}}\right) .
$$

Now using the non-Archimedean triangle inequality

$$
\lambda_{H\left(\eta^{\prime}\right)}(\alpha+\beta) \leq \max \left\{\lambda_{H\left(\eta^{\prime}\right)}(\alpha), \lambda_{H\left(\eta^{\prime}\right)}(\beta)\right\},
$$


we estimate the level

$$
\lambda_{H\left(\eta^{\prime}\right)}\left(h_{\mathcal{H}_{\eta_{0} \eta} \#(\rho ; R)} \mathcal{H}_{\eta \eta^{\prime}}\left(\alpha_{0}\right)+\partial_{J\left(\eta^{\prime}\right), H\left(\eta^{\prime}\right)} \circ H_{\left(\bar{j}_{\eta_{0} \eta^{\prime}}, \overline{\mathcal{H}}_{\eta_{0} \eta^{\prime}} ; \bar{\rho}\right)}\left(\alpha_{0}\right)\right) .
$$

For the first term of (8.14), we have (8.11). For the second term, we recall Theorem 6.7 and Proposition 7.4 which imply

$$
\begin{aligned}
& \lambda_{H\left(\eta^{\prime}\right)}\left(\partial_{J\left(\eta^{\prime}\right), H\left(\eta^{\prime}\right)} \circ H_{\left(\bar{j}_{\eta_{0} \eta^{\prime}}, \overline{\mathcal{H}}_{\eta_{0} \eta^{\prime}} ; \bar{\rho}\right)}\left(\alpha_{0}\right)\right) \\
\leq & \lambda_{H\left(\eta^{\prime}\right)}\left(H_{\left(\bar{j}_{\eta_{0} \eta^{\prime}}, \overline{\mathcal{H}}_{\eta_{0} \eta^{\prime}} ; \bar{\rho}\right)}\left(\alpha_{0}\right)\right)-A_{\left(\mathcal{H}, j^{\eta_{0}} ; \eta_{0}\right)}^{1}
\end{aligned}
$$

for all Floer points $\eta^{\prime}$ satisfying

$$
\left|\eta^{\prime}-\eta_{0}\right|<\delta \quad \text { and } \quad d\left(\eta^{\prime}, \mathcal{S i n g}(\mathcal{H})\right)>\delta
$$

for some $\delta=\delta\left(\mathcal{H}, j, \eta_{0}\right)$. Therefore combining (8.11)-(8.15), we derive (8.16)

$$
\begin{aligned}
\mu\left(\eta^{\prime}\right) & =\lambda_{H\left(\eta^{\prime}\right)}\left(h_{\left(\eta_{0} \eta^{\prime} ; \rho_{0}\right)}\left(\alpha_{0}\right)\right) \\
& \leq \max \left\{\mu(\eta)+E^{-}\left(\mathcal{H}_{\eta \eta^{\prime}}\right), \lambda_{H\left(\eta_{0}\right)}\left(\alpha_{0}\right)+E\left(\mathcal{H}_{\eta_{0} \eta^{\prime}}\right)-A_{\left(\mathcal{H}, j^{\eta_{0}} ; \eta_{0}\right)}^{1}\right\} .
\end{aligned}
$$

We will study under what conditions, we have

$$
\mu(\eta)+E^{-}\left(\mathcal{H}_{\eta \eta^{\prime}}\right) \geq \lambda_{H\left(\eta_{0}\right)}\left(\alpha_{0}\right)+E\left(\mathcal{H}_{\eta_{0} \eta^{\prime}}\right)-A_{\left(\mathcal{H}, j^{\eta_{0}} ; \eta_{0}\right)}^{1}
$$

and in turn $\mu\left(\eta^{\prime}\right) \leq \mu(\eta)+E^{-}\left(\mathcal{H}_{\eta \eta^{\prime}}\right)$.

We apply the homotopy $\left(\mathcal{H}_{\eta_{0} \eta^{\prime}}^{-1}\right)_{\rho_{0}}$ to $(8.8)$ and obtain

$$
h_{\left(\mathcal{H}_{\eta_{0} \eta^{\prime}}^{-1} ; \rho_{0}\right)} \circ h_{\mathcal{H}_{\eta_{0} \eta} \#(\rho ; R)} \mathcal{H}_{\eta \eta^{\prime}}=h_{\left(\mathcal{H}_{\eta_{0} \eta^{\prime}}^{-1} ; \rho_{0}\right)} \circ h_{\left(\eta \eta^{\prime} ; \rho_{1}\right)} \circ h_{\left(\eta_{0} \eta ; \rho_{0}\right)},
$$

where $\rho=\left(\rho_{0}, \rho_{1}\right)$. It follows from (8.17) that the cycle $h_{\left(\mathcal{H}_{\eta_{0} \eta^{\prime}}^{-1}, \rho_{0}\right)} \circ h_{\left(\eta \eta^{\prime} ; \rho_{1}\right)} \circ$ $h_{\left(\eta_{0} \eta ; \rho_{0}\right)}\left(\alpha_{0}\right)$ is homologous to $\alpha_{0}$ since $h_{\left(\mathcal{H}_{\eta_{0} \eta^{\prime}}^{-1} ; \rho_{0}\right)} \circ h_{\mathcal{H}_{\eta_{0} \eta} \#(\rho ; R)} \mathcal{H}_{\eta \eta^{\prime}}$ is chain homotopic to the identity. Therefore, by the tightness assumption on $\alpha_{0}$, we derive

$$
\lambda_{H\left(\eta_{0}\right)}\left(h_{\left(\mathcal{H}_{\eta_{0} \eta^{\prime}}^{-1} ; \rho_{0}\right)} \circ h_{\left(\eta \eta^{\prime} ; \rho_{1}\right)} \circ h_{\left(\eta_{0} \eta ; \rho_{0}\right)}\left(\alpha_{0}\right)\right) \geq \lambda_{H\left(\eta_{0}\right)}\left(\alpha_{0}\right) .
$$

Applying Lemma 8.6 to $h_{\left(\mathcal{H}_{\eta_{0} \eta^{\prime}}^{-1} ; \rho_{0}\right)}$ and the cycle $h_{\left(\eta \eta^{\prime} ; \rho_{1}\right)} \circ h_{\left(\eta_{0} \eta ; \rho_{0}\right)}\left(\alpha_{0}\right)$, we obtain

$$
\begin{aligned}
& \lambda_{H\left(\eta_{0}\right)}\left(h_{\left(\mathcal{H}_{\eta_{0} \eta^{\prime}}^{-1} ; \rho_{0}\right)} \circ h_{\left(\eta \eta^{\prime} ; \rho_{1}\right)} \circ h_{\left(\eta_{0} \eta ; \rho_{0}\right)}\left(\alpha_{0}\right)\right) \\
\leq & \lambda_{H\left(\eta^{\prime}\right)}\left(h_{\left(\eta \eta^{\prime} ; \rho_{1}\right)} \circ h_{\left(\eta_{0} \eta ; \rho_{0}\right)}\left(\alpha_{0}\right)\right)+E^{-}\left(\mathcal{H}_{\eta_{0} \eta^{\prime}}^{-1}\right) \\
= & \lambda_{H\left(\eta^{\prime}\right)}\left(h_{\left(\eta \eta^{\prime} ; \rho_{1}\right)} \circ h_{\left(\eta_{0} \eta ; \rho_{0}\right)}\left(\alpha_{0}\right)\right)+E^{+}\left(\mathcal{H}_{\eta_{0} \eta^{\prime}}\right) .
\end{aligned}
$$

Then (8.17)-(8.19) give rise to

$$
\begin{gathered}
\lambda_{H\left(\eta_{0}\right)}\left(h_{\left(\eta \eta^{\prime} ; \rho_{1}\right)} \circ h_{\left(\eta_{0} \eta ; \rho_{0}\right)}\left(\alpha_{0}\right)\right) \geq \lambda_{H\left(\eta_{0}\right)}\left(\alpha_{0}\right)-E^{+}\left(\mathcal{H}_{\eta_{0} \eta^{\prime}}\right) \\
\geq \lambda_{H(\eta)}\left(h_{\left(\eta_{0} \eta ; \rho_{0}\right)}\left(\alpha_{0}\right)\right)-E^{-}\left(\mathcal{H}_{\eta_{0} \eta}\right)-E^{+}\left(\mathcal{H}_{\eta_{0} \eta^{\prime}}\right),
\end{gathered}
$$


where we applied Lemma 8.6 for the latter inequality. Now after rewriting (8.12) as

$$
h_{\mathcal{H}_{\eta_{0} \eta} \#(\rho ; R)} \mathcal{H}_{\eta \eta^{\prime}}\left(\alpha_{0}\right)=h_{\left(\eta_{0} \eta^{\prime} ; \rho_{0}\right)}\left(\alpha_{0}\right)-\partial_{\left(J\left(\eta^{\prime}\right), H\left(\eta^{\prime}\right)\right)} \circ H_{\left(\bar{j}_{\eta_{0} \eta^{\prime}}, \overline{\mathcal{H}}_{\eta_{0} \eta^{\prime}} ; \bar{\rho}\right)}\left(\alpha_{0}\right),
$$

we derive, from this, (8.8), (8.14) and (8.15), that we have

$$
\begin{aligned}
& \lambda_{H\left(\eta^{\prime}\right)}\left(h_{\left(\eta_{0} \eta ; \rho_{0}\right)} \circ h_{\left(\eta \eta^{\prime} ; \rho_{1}\right)}\left(\alpha_{0}\right)\right) \\
= & \lambda_{H\left(\eta^{\prime}\right)}\left(h_{\mathcal{H}_{\eta_{0} \eta} \#(\rho ; R)} \mathcal{H}_{\eta \eta^{\prime}}\left(\alpha_{0}\right)\right) \\
\leq & \max \left\{\lambda_{H\left(\eta^{\prime}\right)}\left(h_{\left(\eta_{0} \eta^{\prime} ; \rho_{0}\right)}\left(\alpha_{0}\right)\right), \lambda_{H\left(\eta_{0}\right)}\left(\alpha_{0}\right)+E\left(\mathcal{H}_{\eta_{0} \eta^{\prime}}\right)-A_{\left(\mathcal{H}, j^{\eta_{0}} ; \eta_{0}\right)}^{1}\right\}
\end{aligned}
$$

as in (8.16). On the other hand, we derive, from (8.20) and (8.22),

$$
\begin{aligned}
& \max \left\{\lambda_{H\left(\eta^{\prime}\right)}\left(h_{\left(\eta_{0} \eta^{\prime} ; \rho_{0}\right)}\left(\alpha_{0}\right)\right), \lambda_{H\left(\eta_{0}\right)}\left(\alpha_{0}\right)+E\left(\mathcal{H}_{\eta_{0} \eta^{\prime}}\right)-A_{\left(\mathcal{H}, j^{\eta_{0}} ; \eta_{0}\right)}^{1}\right\} \\
\geq & \lambda_{H\left(\eta_{0}\right)}\left(\alpha_{0}\right)-E^{+}\left(\mathcal{H}_{\eta_{0} \eta^{\prime}}\right) .
\end{aligned}
$$

Now we choose $\delta_{5}=\delta_{5}\left(\mathcal{H}, j^{\eta_{0}}, \eta_{0}\right)>0$ so that

$$
E\left(\mathcal{H}_{\eta_{0} \eta^{\prime}}\right)-A_{\left(\mathcal{H}, j^{\eta_{0}} ; \eta_{0}\right)}^{1}<-E^{+}\left(\mathcal{H}_{\eta_{0} \eta^{\prime}}\right)
$$

i.e.,

$$
E\left(\mathcal{H}_{\eta_{0} \eta^{\prime}}\right)+E^{+}\left(\mathcal{H}_{\eta_{0} \eta^{\prime}}\right)<A_{\left(\mathcal{H}, j^{\eta_{0}} ; \eta_{0}\right)}^{1}
$$

holds for any $\eta, \eta^{\prime} \in\left(\eta_{0}-\delta_{5}, \eta_{0}-\delta_{5}\right)$. We would like to emphasize that we can choose $\delta_{5}$ so that it satisfies (8.24) and depends only on $\left(\mathcal{H}, j, \eta_{0}\right)$. With this choice of $\delta_{5}$ made, we have

$$
\lambda_{H\left(\eta_{0}\right)}\left(\alpha_{0}\right)+E\left(\mathcal{H}_{\eta_{0} \eta^{\prime}}\right)-A_{\left(\mathcal{H}, j^{\eta_{0}} ; \eta_{0}\right)}^{1}<\lambda_{H\left(\eta_{0}\right)}\left(\alpha_{0}\right)-E^{+}\left(\mathcal{H}_{\eta_{0} \eta^{\prime}}\right) .
$$

Then (8.23) and (8.25) imply

$$
\lambda_{H\left(\eta^{\prime}\right)}\left(h_{\left(\eta_{0} \eta^{\prime} ; \rho_{0}\right)}\left(\alpha_{0}\right)\right) \geq \lambda_{H\left(\eta_{0}\right)}\left(\alpha_{0}\right)-E^{+}\left(\mathcal{H}_{\eta_{0} \eta^{\prime}}\right)
$$

Now combining (8.16), (8.25) and (8.26), we first obtain

$$
\begin{aligned}
& \max \left\{\mu(\eta)+E^{-}\left(\mathcal{H}_{\eta \eta^{\prime}}\right), \lambda_{H\left(\eta_{0}\right)}\left(\alpha_{0}\right)+E\left(\mathcal{H}_{\eta_{0} \eta^{\prime}}\right)-A_{\left(\mathcal{H}, j^{\eta_{0}} ; \eta_{0}\right)}^{1}\right\} \\
= & \mu(\eta)+E^{-}\left(\mathcal{H}_{\eta \eta^{\prime}}\right)
\end{aligned}
$$

which in turn implies

$$
\mu\left(\eta^{\prime}\right) \leq \mu(\eta)+E^{-}\left(\mathcal{H}_{\eta \eta^{\prime}}\right) .
$$

Next we compare the homotopy $\mathcal{H}_{\eta_{0} \eta}$ and $\mathcal{H}_{\eta \eta^{\prime}}^{-1} \# \mathcal{H}_{\eta_{0} \eta^{\prime}}$. Recall $\eta^{\prime}>\eta$ and $\mathcal{H}_{\eta^{\prime} \eta}=\mathcal{H}_{\eta \eta^{\prime}}^{-1}$ by Definition 3.8. Again we will have the identity

$$
h_{\left(\mathcal{H}^{-1}\right)_{\eta \eta^{\prime}} \#_{(\rho ; R)} \mathcal{H}_{\eta_{0} \eta^{\prime}}}=h_{\eta^{\prime} \eta ; \rho_{1}} \circ h_{\eta_{0} \eta^{\prime} ; \rho_{0}}
$$

for a sufficiently large $R$. Now (8.11) is replaced by

$$
\lambda_{H(\eta)}\left(h_{\left(\mathcal{H}^{-1}\right)_{\eta \eta^{\prime}} \#_{(\rho ; R)} \mathcal{H}_{\eta_{0} \eta^{\prime}}}\left(\alpha_{0}\right)\right) \leq \mu\left(\eta^{\prime}\right)+E^{-}\left(\mathcal{H}_{\eta \eta^{\prime}}^{-1}\right)=\mu\left(\eta^{\prime}\right)+E^{+}\left(\mathcal{H}_{\eta \eta^{\prime}}\right) .
$$

Here we would like to remark that $\left(\mathcal{H}^{-1}\right)_{\eta \eta^{\prime}} \#_{(\rho ; R)} \mathcal{H}_{\eta_{0} \eta^{\prime}}$ is still an elongation of $\mathcal{H}_{\eta_{0} \eta}$ but by a non-monotone cut off function. 
By repeating the arguments above with $\mathcal{H}_{\eta \eta^{\prime}}$ replaced by $\mathcal{H}_{\eta \eta^{\prime}}^{-1}$, we obtain

$$
\mu(\eta) \leq \mu\left(\eta^{\prime}\right)+E^{+}\left(\mathcal{H}_{\eta \eta^{\prime}}\right) .
$$

Combining (8.27) and (8.28), we have obtained

$$
-E^{+}\left(\mathcal{H}_{\eta \eta^{\prime}}\right) \leq \mu\left(\eta^{\prime}\right)-\mu(\eta) \leq E^{-}\left(\mathcal{H}_{\eta \eta^{\prime}}\right) .
$$

This is indeed an inequality stronger than (8.7) and in particular proves (8.7). The cases other than that of $\eta_{0}<\eta<\eta^{\prime}$ can be handled by the same arguments if we replace the sub-homotopy $\mathcal{H}_{s s^{\prime}}$ by $\left(\mathcal{H}^{-1}\right)_{s^{\prime} s}$ every time the reverse inequality $s>s^{\prime}$ appears in the proof. This explains appearance of $E$ in general instead of $E^{-}$or $E^{+}$in (8.7). We leave the details to the readers. This finishes the proof of Lemma 8.5 and hence Proposition 8.4.

Remark 8.7. In fact, an examination of the above proof combined with the discussion above Proposition 3.8 shows that the map $\eta \mapsto \lambda_{H(\eta)}\left(h_{\left(\eta_{0} \eta ; \rho_{0}\right)}\left(\alpha_{0}\right)\right)$ is differentiable such that its derivative depends only on the periodic orbit but not on its liftings and is given by

$$
-\int_{0}^{1} \frac{\partial H(\eta)}{\partial \eta}\left(t, z_{\eta}(t)\right) d t
$$

where $z_{\eta}$ is a periodic orbit with $\left[z_{\eta}, w_{\eta}\right]$ is a peak of the cycle $h_{\left(\eta_{0} \eta ; \rho_{0}\right)}\left(\alpha_{0}\right)$.

Next we note that there is a canonical one-one correspondence

$$
\operatorname{Per}\left(H\left(\eta_{0}\right)\right) \leftrightarrow \operatorname{Per}(H(\eta))
$$

for any $\eta \in\left(\eta_{0}-\delta, \eta_{0}+\delta\right)$ for some $\delta=\delta\left(\mathcal{H}, \eta_{0}\right)>0$. We denote the corresponding family by $z_{k}(\eta)$ for $k=1, \ldots, \#\left(\operatorname{Per}\left(H\left(\eta_{0}\right)\right)\right.$. This map in turn induces a one-one correspondence

$$
\text { Crit } \mathcal{A}_{H\left(\eta_{0}\right)} \leftrightarrow \text { Crit } \mathcal{A}_{H\left(\eta_{0}\right)}
$$

given by

$$
\left[z_{k}\left(\eta_{0}\right), w_{k}\left(\eta_{0}\right)\right] \leftrightarrow\left[z_{k}(\eta), w_{k}\left(\eta_{0}\right) \# u_{z_{k}\left(\eta_{0}\right) z_{k}(\eta)}^{c a n}\right] .
$$

For the simplicity of notation, we denote $u_{\eta_{0} \eta, k}^{c a n}=u_{z_{k}\left(\eta_{0}\right) z_{k}(\eta)}^{c a n}$. By construction of $h_{\left(\eta_{0} \eta ; \rho_{0}\right)}$, we then have

$$
h_{\left(\eta_{0} \eta ; \rho_{0}\right)}\left(\left[z_{k}\left(\eta_{0}\right), w_{k}\left(\eta_{0}\right)\right]\right)=\left[z_{k}(\eta), w_{k}\left(\eta_{0}\right) \# u_{\eta_{0} \eta, k}^{c a n}\right]
$$

modulo any filtration order as want, by choosing $\delta$ smaller if necessary. We recall the identity $h_{\left(\eta \eta ; \rho_{0}\right)} \equiv i d$ in the chain level. We will always assume this holds for the rest of the proof.

We start with the following result which is of independent interest. A similar statement has been an important ingredient of Usher's algebraic proof of Theorem II [22]. It was also proved in [9] in the context of Lagrangian intersection Floer cohomology by a purely algebraic way, but with $\Lambda_{0, n o v}^{(0)}$ the (positive) universal Novikov ring. (See [9, Proposition 26.9].) Since we use the Novikov ring $\Lambda_{\omega}$, which does not have this positivity property, we cannot deduce this 
result from [9, Proposition 26.9]. Here we instead give a simple geometric proof based on the basic fact, Lemma 8.9 below, on the Floer boundary operator for a small Morse function.

Proposition 8.8. There exists a constant $c>0$ depending only on $\mathcal{H}$ but independent of $\eta_{0}$ or $\lambda$ such that

$$
\partial\left(C F\left(H\left(\eta_{0}\right)\right) \cap C F^{\lambda}\left(H\left(\eta_{0}\right)\right) \subset \partial\left(C F^{\lambda+c}\left(H\left(\eta_{0}\right)\right)\right)\right.
$$

for all $\lambda \in \mathbb{R}$.

Proof. Denote $H=H\left(\eta_{0}\right)$ and $\partial_{(H, J)}=\partial_{H}$ in this proof for the simplicity of notations. We first recall the following well-known result for a small Morse function $\epsilon f$. See [7], [18] for its proof.

Lemma 8.9. Let $f$ be a Morse function and $\epsilon>0$ be a sufficiently small so that so that $\partial_{\epsilon f}=\partial^{\text {Morse }}(-\epsilon f) \otimes \Lambda_{\omega}$. Let $c_{0}=\epsilon \max |f|$. In particular, we have

$$
\partial(C F(\epsilon f)) \cap C F^{\lambda}(\epsilon f) \subset \partial\left(C F_{k}^{\lambda+c_{0}}(\epsilon f)\right) .
$$

We now connect the given Cerf homotopy $\mathcal{H}$ by another fixed homotopy from $\epsilon f$ and $H^{0}$ and denote the join homotopy again by $\mathcal{H}$ which now connects $\epsilon f$ to $H$. Let $\gamma \in \partial(C F(H)) \cap C F^{\lambda}(H)$. We transfer $\gamma$ to $C F(\epsilon f)$ and consider $h_{\eta_{0} 0}(\gamma)$. Note

$$
\lambda_{\epsilon f}\left(h_{\eta_{0} 0}(\gamma)\right) \leq \lambda_{H}(\gamma)+E^{+}(\mathcal{H}) \leq \lambda+E^{+}(\mathcal{H}) .
$$

Therefore it follows from Lemma A.4 that there exists a chain $\beta_{0} \in C F(\epsilon f)$ such that

$$
h_{\eta_{0} 0}(\gamma)=\partial_{\epsilon f}\left(\beta_{0}\right), \quad \lambda_{\epsilon f}\left(\beta_{0}\right) \leq \lambda+E^{+}(\mathcal{H})+\epsilon \max |f| .
$$

We estimate

(8.30) $\lambda_{H}\left(h_{0 \eta_{0}}\left(\beta_{0}\right)\right) \leq \lambda+E^{+}(\mathcal{H})+\epsilon \max |f|+E^{-}(\mathcal{H})=\lambda+E(\mathcal{H})+\epsilon \max |f|$.

We compute $\partial_{H}\left(h_{0 \eta_{0}}\left(\beta_{0}\right)\right)$

$$
\partial_{H}\left(h_{0 \eta_{0}}\left(\beta_{0}\right)\right)=h_{0 \eta_{0}}\left(\partial_{\epsilon f}\left(\beta_{0}\right)\right)=h_{0 \eta_{0}}\left(h_{\eta_{0} 0}(\gamma)\right) .
$$

By the chain homotopy formula, we obtain

$$
h_{0 \eta_{0}} \circ h_{\eta_{0} 0}(\gamma)=\gamma+\partial_{H}\left(\bar{H}_{\mathcal{H}_{\eta_{0} 0} \# \mathcal{H}_{0 \eta_{0}}}(\gamma)\right)
$$

and hence

$$
\partial_{H}\left(h_{0 \eta_{0}}\left(\beta_{0}\right)-\bar{H}_{\mathcal{H}_{\eta_{0} 0} \# \mathcal{H}_{0 \eta_{0}}}(\gamma)\right)=\gamma .
$$

We consider $\beta=h_{0 \eta_{0}}\left(\beta_{0}\right)-\bar{H}_{\mathcal{H}_{\eta_{0} 0}}(\gamma)$ and estimate its level

$$
\lambda_{H}(\beta) \leq \max \left\{\lambda_{H}\left(h_{0 \eta_{0}}\left(\beta_{0}\right)\right), \lambda_{H}\left(\bar{H}_{\mathcal{H}_{\eta_{0} 0} \# \mathcal{H}_{0 \eta_{0}}}(\gamma)\right)\right\} .
$$

But we have the estimates

$$
\lambda_{H}\left(h_{0 \eta_{0}}\left(\beta_{0}\right)\right) \leq \lambda+E(\mathcal{H})+\epsilon \max |f|
$$


from (8.30) and

$$
\lambda_{H}\left(\bar{H}_{\mathcal{H}_{\eta_{0} 0} \# \mathcal{H}_{0 \eta_{0}}}(\gamma)\right) \leq \lambda_{H}(\gamma)+E(\mathcal{H}) \leq \lambda+E(\mathcal{H})
$$

from (5.25). Hence by taking $c=E(\mathcal{H})+\epsilon \max |f|$, we have finished the proof.

Now we are ready to give the proof of Theorem 8.3.

Proof of Theorem 8.3. Fix a positive constant $\delta<\min \lambda_{1} / 2$. Proposition 8.8 implies that any element from the submodule $\partial\left(C F\left(H\left(\eta_{0}\right)\right) \cap C F^{\lambda}\left(H\left(\eta_{0}\right)\right)\right.$ with $\lambda=\rho\left(H\left(\eta_{0}\right) ; a\right)+\delta$ can be represented by $\partial_{H\left(\eta_{0}\right)}(\beta)$ with

$$
\lambda_{H\left(\eta_{0}\right)}(\beta) \leq \rho\left(H\left(\eta_{0}\right) ; a\right)+\delta+c .
$$

Now we choose a tight cycle $\alpha_{0}$ and its decomposition

$$
\alpha_{0}=\operatorname{peak}\left(\alpha_{0}\right)+\widetilde{\alpha}_{0} .
$$

We denote the gap of the operator $\partial_{\left(H\left(\eta_{0}\right), J\left(\eta_{0}\right)\right)}$ by $\lambda_{1}=\lambda_{1}\left(\eta_{0}\right):=A_{\left(H\left(\eta_{0}\right), J\left(\eta_{0}\right)\right)}$ given in Proposition 6.2.

We then consider the transferred cycle $h_{\eta_{0} \eta}\left(\alpha_{0}\right):=\alpha(\eta)$ for $\eta$ sufficiently close to $\eta_{0}$. We will show the following two alternatives: there exists $\delta_{4}=$ $\delta_{4}\left(\mathcal{H}, j, a ; \eta_{0}\right)$ such that

(1) either $h_{\eta_{0} \eta}\left(\alpha_{0}\right)$ is tight for $H(\eta)$ for all $\eta \in\left(\eta_{0}-\delta_{4}, \eta_{0}+\delta_{4}\right)$,

(2) or $h_{\eta_{0} \eta}\left(\alpha_{0}\right)$ is not tight and there exist tight cycles $\alpha_{ \pm}$for $H\left(\eta_{0}\right)$ such that $h_{\eta_{0} \eta}\left(\alpha_{ \pm}\right)$are tight on $\left(\eta_{0}-\delta_{4}, \eta_{0}\right]$ and on $\left[\eta_{0}, \eta_{0}+\delta_{4}\right)$ respectively.

In the second case, $\alpha_{-}$and $\alpha_{+}$could be different cycles.

Now suppose that the cycles $h_{\eta_{0} \eta}\left(\alpha_{0}\right)$ is not tight for $H(\eta)$ at least in one direction, say, for $\eta<\eta_{0}$. We will try to find another tight cycle $\alpha_{-}$at $\eta_{0}$ for which the second alternative above holds.

Since $h_{\eta_{0} \eta}\left(\alpha_{0}\right)$ is not tight, there exists a chain $\beta(\eta)$ such that

$$
\lambda_{H(\eta)}\left(h_{\eta_{0} \eta}\left(\alpha_{0}\right)+\partial_{H(\eta)}(\beta(\eta))\right)<\lambda_{H(\eta)}\left(h_{\eta_{0} \eta}\left(\alpha_{0}\right)\right) .
$$

This in particular implies that $\partial_{H(\eta)}(\beta(\eta)) \operatorname{kills}$ peak $\left(h_{\eta_{0} \eta}\left(\alpha_{0}\right)\right)$.

Since we have

$$
\lambda_{H(\eta)}(\beta(\eta)) \leq \lambda_{H\left(\eta_{0}\right)}\left(h_{\eta \eta_{0}}(\beta(\eta))+C_{(\mathcal{H}, j)}\left(\left|\eta-\eta_{0}\right|\right)\right.
$$

we may assume by Proposition 8.8 that

$$
\lambda_{H(\eta)}(\beta(\eta)) \leq \rho\left(H\left(\eta_{0}\right) ; a\right)+2 \delta+c
$$

if we choose $\eta$ so that $C_{(\mathcal{H}, j)}\left(\left|\eta-\eta_{0}\right|\right)<\delta$, where the constant $C_{(\mathcal{H}, j)}(r)$ is the one given in Proposition 6.6.

If $h_{\eta \eta_{0}}\left(\operatorname{peak}\left(\alpha_{0}\right)\right)=\partial_{H(\eta)} \gamma^{\prime}$ for some $\gamma^{\prime}$, applying the homotopy formula between $h_{\eta_{0} \eta} \circ h_{\eta \eta_{0}}$ and the identity, we derive

$$
\operatorname{peak}\left(\alpha_{0}\right)=\partial_{H\left(\eta_{0}\right)}\left(h_{\eta_{0} \eta}\left(\gamma^{\prime}\right)\right)+\partial_{H\left(\eta_{0}\right)}\left(\bar{H} \operatorname{peak}\left(\alpha_{0}\right)\right)+\bar{H}\left(\partial_{H\left(\eta_{0}\right)}\left(\operatorname{peak}\left(\alpha_{0}\right)\right)\right) \text {. }
$$


It is easy to show that the levels of the last two terms are less than equal to

$$
\lambda_{H\left(\eta_{0}\right)}\left(\operatorname{peak}\left(\alpha_{0}\right)\right)-\frac{2 \lambda_{1}\left(\eta_{0}\right)}{3}+E\left(\mathcal{H}_{\eta_{0} \eta}\right)<\lambda_{H\left(\eta_{0}\right)}\left(\operatorname{peak}\left(\alpha_{0}\right)\right),
$$

if we choose $\left|\eta_{0}-\eta\right|$ so small that $E\left(\mathcal{H}_{\eta_{0} \eta}\right) \leq \frac{\lambda_{1}}{3}$. Therefore $\partial_{H\left(\eta_{0}\right.} \gamma$ with $\gamma=h_{\eta_{0} \eta}\left(\gamma^{\prime}\right)$ kills peak $\left.\left(\alpha_{0}\right)\right)$ modulo terms of level less than $\lambda_{H\left(\eta_{0}\right)}\left(\operatorname{peak}\left(\alpha_{0}\right)\right)$. Hence we have

$$
\lambda_{H\left(\eta_{0}\right)}\left(\alpha_{0}-\partial_{H\left(\eta_{0}\right)} \gamma\right)<\lambda_{H\left(\eta_{0}\right)}\left(\alpha_{0}\right)
$$

which violates tightness of $\alpha_{0}$. Therefore we may assume that $h_{\eta_{0} \eta}\left(\operatorname{peak}\left(\alpha_{0}\right)\right)$ is not a boundary.

Furthermore if $\beta(\eta)$ has a generator $\left[z^{\prime}, w^{\prime}\right]$ not connected to $h_{\eta_{0} \eta}\left(\operatorname{peak}\left(\alpha_{0}\right)\right)$, i.e., if $\partial_{H(\eta)}\left(\left[z^{\prime}, w^{\prime}\right]\right)$ is not contributed by any generator thereof, we can safely replace $\beta(\eta)$ by $\beta(\eta)-a^{\prime}\left[z^{\prime}, w^{\prime}\right]$ for some $a^{\prime} \in \mathbb{Q}$ to get rid of the generator $\left[z^{\prime}, w^{\prime}\right]$ without increasing the level of $h_{\eta_{0} \eta}\left(\alpha_{0}\right)+\partial_{H(\eta)}(\beta(\eta))$ : Since $h_{\eta_{0} \eta}\left(\operatorname{peak}\left(\alpha_{0}\right)\right)$ is not a boundary and since the peak of $h_{\eta_{0} \eta}\left(\alpha_{0}\right)$ is contained in $h_{\eta_{0} \eta}\left(\operatorname{peak}\left(\alpha_{0}\right)\right)$, this $\partial \beta(\eta)$ must have a non-zero remainder, after it kills peak $\left(h_{\eta_{0} \eta}\left(\alpha_{0}\right)\right)$. The level of the remainder must be lower than $\lambda_{H(\eta)}\left(h_{\eta_{0} \eta}\left(\alpha_{0}\right)\right)$ by $(8.31)$. Therefore we may assume that all the generators of $\beta(\eta)$ is connected to some generator of $h_{\eta_{0} \eta}\left(\operatorname{peak}\left(\alpha_{0}\right)\right)$. This in turn implies that all the generators of $h_{\eta_{0} \eta}^{-1}(\beta(\eta))$ are also connected to peak $\left(\alpha_{0}\right)$ by (8.29).

We also derive from $(8.31)$

$$
\lambda_{H\left(\eta_{0}\right)}\left(h_{\eta_{0} \eta}^{-1}(\beta(\eta))<\rho\left(H\left(\eta_{0}\right) ; a\right)+3 \delta+c .\right.
$$

By the Gromov-Floer compactness, there are only finitely many, say,

$$
N_{0}=N_{0}\left(\mathcal{H}, \eta_{0}, a, \rho\left(H\left(\eta_{0}\right) ; a\right), \rho\left(H\left(\eta_{0}\right) ; a\right)+3 \delta+c\right)
$$

critical points with

$$
\left[z_{1}\left(\eta_{0}\right), w_{1}\left(\eta_{0}\right)\right], \ldots,\left[z_{N_{0}}\left(\eta_{0}\right), w_{N_{0}}\left(\eta_{0}\right)\right]
$$

connected to $\left(\mathcal{A}_{H\left(\eta_{0}\right)}^{-1}\left(\rho\left(H\left(\eta_{0}\right) ; a\right)\right) \cap \operatorname{Crit} \mathcal{A}_{H\left(\eta_{0}\right)}\right.$ (and so those connected to peak $\left.\left(\alpha_{0}\right)\right)$. Since $\alpha_{0}$ is tight, there must exist at least one $\left[z_{\ell}, w_{\ell}\right]$ for which there is another critical point $\left[z_{\ell}^{\prime}, w_{\ell}^{\prime}\right]$ of $\mathcal{A}_{H\left(\eta_{0}\right)}$ such that

$$
\#\left(\mathcal{M}\left(\left[z_{\ell}, w_{\ell}\right],\left[z_{\ell}^{\prime}, w_{\ell}^{\prime}\right]\right) \neq 0\right.
$$

and

$$
\mathcal{A}_{H\left(\eta_{0}\right)}\left(\left[z_{\ell}^{\prime}, w_{\ell}^{\prime}\right]\right) \geq \lambda_{H\left(\eta_{0}\right)}\left(\alpha_{0}\right) .
$$

We divide our discussion into two cases : one for which the cycle $\alpha_{0}^{\prime}:=$ $\alpha_{0}+\partial_{H\left(\eta_{0}\right)}\left(h_{\eta_{0} \eta}^{-1}(\beta(\eta))\right.$ is tight and so

$$
\lambda_{H\left(\eta_{0}\right)}\left(\alpha_{0}\right)=\lambda_{H\left(\eta_{0}\right)}\left(\alpha_{0}^{\prime}\right)
$$

and the other for which $\alpha_{0}^{\prime}$ is not tight and so

$$
\lambda_{H\left(\eta_{0}\right)}\left(\alpha_{0}^{\prime}\right)>\lambda_{H\left(\eta_{0}\right)}\left(\alpha_{0}\right) .
$$

We start with the case (8.35). In this case, the inequality (8.33) must be strict for at least one $\ell$ : Otherwise the cycle $\alpha_{0}^{\prime}$ itself will be tight and so 
belongs to the category (8.34). We recall that there are only finitely many, say $N_{0}=N_{0}\left(H\left(\eta_{0}\right), a\right)$, critical points of $\mathcal{A}_{H\left(\eta_{0}\right)}$ at the level $y_{0}=\rho\left(H\left(\eta_{0}\right) ; a\right)$. This number depends only on $H\left(\eta_{0}\right)$ and $a$. And we note that there are at most finitely many $\left[z_{\ell}^{\prime}\left(\eta_{0}\right), z_{\ell}^{\prime}\left(\eta_{0}\right)\right]$ connected to $\left[z_{\ell}\left(\eta_{0}\right), z_{\ell}\left(\eta_{0}\right)\right]$, say,

$$
N_{1}(\ell)=N_{1}\left(\mathcal{H}, \eta_{0}, a, \rho\left(H\left(\eta_{0}\right) ; a\right), \rho\left(H\left(\eta_{0}\right) ; a\right)+3 \delta+c ; \ell\right)
$$

for each $\ell$. Therefore if we define

$$
N_{2}=\sum_{\ell=1}^{N_{0}} N_{1}(\ell)
$$

there will be at most $N_{2}$ such $\left[z_{\ell}^{\prime}, w_{\ell}^{\prime}\right]$ in total. Furthermore by the definition of the gap $\lambda_{1}\left(\eta_{0}\right)$ of the boundary map $\partial_{H\left(\eta_{0}\right)}$, we have

$$
\rho\left(H\left(\eta_{0}\right) ; a\right)+\lambda_{1}\left(\eta_{0}\right) \leq \lambda_{\left(H\left(\eta_{0}\right)\right)}\left(\left[z_{\ell}\left(\eta_{0}\right), w_{\ell}\left(\eta_{0}\right)\right]\right) \leq \rho\left(H\left(\eta_{0}\right) ; a\right)+3 \delta+c .
$$

Then from (8.35) and from the finiteness of such $\left[z_{\ell}^{\prime}, w_{\ell}^{\prime}\right]$ connected to $\left[z_{\ell}, w_{\ell}\right]$ which in turn connected to $\left[z_{0}, w_{0}\right]$ a generator of peak $\left(\alpha_{0}\right)$, we derive that there must be a gap denoted by

$$
\lambda_{2}\left(\eta_{0}\right)=\lambda_{2}\left(\mathcal{H}, \eta_{0}, a, \rho\left(H\left(\eta_{0}\right) ; a\right), \rho\left(H\left(\eta_{0}\right) ; a\right)+3 \delta+c\right)
$$

such that

$$
\mathcal{A}_{H\left(\eta_{0}\right)}\left(\left[z_{\ell}^{\prime}, w_{\ell}^{\prime}\right]\right)>\lambda_{H\left(\eta_{0}\right)}\left(\alpha_{0}\right)+\lambda_{2}
$$

for all such $\left[z_{\ell}^{\prime}, w_{\ell}^{\prime}\right]$. (In Lemma 8.10 below, we will in fact show that we can choose $\lambda_{2}$ depending only on $(\mathcal{H}, j)$ and $3 \delta+c$.)

By nondegeneracy of $H\left(\eta_{0}\right)$ and finiteness of \# $\operatorname{Per}\left(H\left(\eta_{0}\right)\right)$, we can choose a sufficiently small $r:=\left|\eta-\eta_{0}\right|>0$ so that

$$
C_{(\mathcal{H}, j)}(r)<\min \left\{\frac{\lambda_{2}}{4}, \lambda_{1}-2 C_{(\mathcal{H}, j)}(r)\right\} .
$$

Furthermore we derive from (8.36) that for any such $\left[z_{\ell}^{\prime}\left(\eta_{0}\right), w_{\ell}^{\prime}\left(\eta_{0}\right)\right]$ the action of transferred critical points $\left[z_{\ell}^{\prime}(\eta), w_{\ell}^{\prime}(\eta)\right]$ satisfies

$$
\mathcal{A}_{H(\eta)}\left(\left[z_{\ell}^{\prime}(\eta), w_{\ell}^{\prime}(\eta)\right]\right) \geq \mathcal{A}_{H(\eta)}\left(\left[z_{0}(\eta), w_{0}(\eta)\right]\right)+\frac{\lambda_{2}}{2}=\lambda_{H\left(\eta_{0}\right)}\left(\alpha_{0}\right)+\frac{\lambda_{2}}{2}
$$

by the choice of $\eta$. This then implies that as long as $\left|\eta-\eta_{0}\right| \leq r$ as above in (8.37), we estimate the level of the cycle $h_{\eta_{0} \eta}\left(\alpha_{0}\right)$ as

$$
\lambda_{H(\eta)}\left(h_{\eta_{0} \eta}\left(\alpha_{0}\right)+\partial \beta(\eta)\right) \geq \lambda_{H\left(\eta_{0}\right)}\left(\alpha_{0}\right)+\frac{\lambda_{2}}{2}>\lambda_{H(\eta)}\left(h_{\eta_{0} \eta}(\alpha(\eta)) .\right.
$$

This contradicts to the hypothesis (8.31). This finishes the proof by setting $\delta_{4}=r$ for the case $(8.35)$.

For the case $(8.34)$, the point $\left(\eta_{0}, y_{0}\right)$ with $y_{0}=\lambda_{H\left(\eta_{0}\right)}\left(\alpha_{0}\right)$ is a nondegenerate crossing in the bifurcation diagram of $\mathcal{H}$. By the Cerf property, the two continuous functions

$$
\begin{aligned}
& \mu_{1}(\eta):=\lambda_{H\left(\eta_{0}\right)}\left(h_{\eta_{0} \eta}\left(\alpha_{0}\right)\right) \\
& \mu_{2}(\eta):=\lambda_{H\left(\eta_{0}\right)}\left(h_{\eta_{0} \eta}\left(\alpha_{0}^{\prime}\right)\right)
\end{aligned}
$$


provide the two possible branches at $\left(\eta_{0}, y_{0}\right)$. By applying the above consideration to $\alpha_{0}^{\prime}$ instead of $\alpha_{0}, \alpha_{0}$ must belong to the category (1) in the above alternatives and so its transferred cycles $h_{\eta_{0} \eta}\left(\alpha_{0}^{\prime}\right)$ must be tight. This finishes the proof.

For the later purpose, we provide a precise description of the above constant $\lambda_{2}$ in the following lemma and its lower semi-continuity property.

Lemma 8.10. Let $K>0$ and define the constant $\lambda_{2}(\eta ; K)$ as follows: for $\eta \notin \operatorname{Sing}(\mathcal{H})$,

$$
\begin{aligned}
\lambda_{2}(\eta ; K):= & \inf _{z, C, C^{\prime}}\left\{E_{(H(\eta), J(\eta))}(u)-E_{(H(\eta), J(\eta))}\left(u^{\prime}\right) \mid u \in \mathcal{M}(J, H ; z,(\cdot) ; C),\right. \\
& u^{\prime} \in \mathcal{M}\left(J, H ; z,(\cdot) ; C^{\prime}\right), 0<E_{(H(\eta), J(\eta))}(u), E_{(H(\eta), J(\eta))}\left(u^{\prime}\right) \leq K, \\
& \left.E_{(H(\eta), J(\eta))}(u)-E_{(H(\eta), J(\eta))}\left(u^{\prime}\right)>0, \mu_{H(\eta)}(C)=\mu_{H(\eta)}\left(C^{\prime}\right)=1\right\}
\end{aligned}
$$

and for $\eta \in \operatorname{Sing}(\mathcal{H})$ by the same except that we add the requirement that at least one of the $\omega$-limits $(\cdot)$ of $u, u^{\prime}$ is not of the form $z^{-}$. Then the function $\eta \in[0,1] \mapsto \lambda_{2}(\eta ; K)$ is lower semi-continuous for each fixed $K$. In particular we have

$$
\lambda_{2, K}:=\min _{\eta \in[0,1]} \lambda_{2}(\eta ; K)>0
$$

Proof. This is a consequence of Gromov-Floer compactness which we can apply as in the proofs of Propositions 6.1 and 6.2 using the following ingredients:

(1) finiteness of \#(Per $H(\eta))$ for each $\eta$,

(2) finiteness of homotopy classes of $u, u^{\prime}$ with the same $\alpha$-limit under the given energy bounds,

(3) and finally by isolatedness of $\mathcal{M}(z,(\cdot) ; C)$ when $\mu_{H}(C)=1$.

\section{$\S 9$. Proof of the nondegenerate spectrality}

Finally we are ready to prove Theorem II, which we reiterate here. Let $H$ be a nondegenerate Hamiltonian and

$$
a=\sum a_{A} q^{-A}, \quad a_{A} \in H^{*}(M)
$$

be a non-zero quantum cohomology class.

Theorem 9.1. Let $H$ be any nondegenerate one-periodic Hamiltonian and $J$ be such that $(H, J)$ is Floer regular. Then for any nonzero quantum cohomology class a, the mini-max value $\rho(H ; a)$ is realized by the level of a tight Floer cycle of $(H, J)$.

Besides the preparatory materials proven in Section 3-7, our proof of Theorem II also exploits the following two ingredients in an essential way:

(1) $\rho(\epsilon f ; a)$ is homologically essential for any Morse function if $\epsilon>0$ is sufficiently small, 
(2) The mini-max value $\rho(H ; a)$ is tied to a nontrivial topological property, not an accidental critical value.

Now we fix a Morse function $f$ and a Cerf homotopy

$$
\mathcal{H} \in \mathcal{P}^{\operatorname{Cerf}}\left(\mathcal{H}_{m}(M) ; \epsilon f, H\right)
$$

satisfying the properties described in Section 7,

$$
\mathcal{H}=\{H(\eta)\}_{0 \leq \eta \leq 1},
$$

such that

$$
H(0)=\epsilon f, \quad H(1)=H .
$$

For simplicity, without loss of any generality, we will assume that the values of the Morse function $f$ at critical points are all distinct.

We also consider the set of $j=\{J(\eta)\}_{0 \leq \eta \leq 1}$ lying in $\mathcal{P}\left(j_{\omega} ; \mathcal{H}\right)$, In particular, by the choice of $j$, the Floer homology $H F_{*}(H(\eta), J(\eta))$ is defined for any $\eta \in I(\mathcal{H}, j)$. We

The proof of Theorem 9.1 will be done by a continuation argument. We define a subset of $[0,1]$

(9.2) $S(\mathcal{H})=\{\eta \in[0,1] \mid \eta$ satisfies one of the following two conditions $\}$ :

(1) when $\eta \in[0,1] \backslash \mathcal{S} \operatorname{ing}(\mathcal{H}), \rho(H(\eta) ; a)$ is homologically essential, i.e., there is a $J$ for which $(H, J)$ is Floer-regular and carries a Floer cycle $\alpha$ with $\rho(H(\eta) ; a)=\lambda_{H}(\alpha)$.

(2) when $\eta \in \mathcal{S i n g}(\mathcal{H})$, there is $j \in \mathcal{P}\left(j_{\omega} ; \mathcal{H}\right)$ such that it carries a sequence of Floer points $\eta_{i} \rightarrow \eta$ for which the mini-max value $\rho\left(H\left(\eta_{i}\right) ; a\right)$ is homologically essential for each $i$.

We will prove $S(\mathcal{H})=[0,1]$ by a continuation argument starting from $H(0)=\epsilon f$, which will in particular prove Theorem II.

\section{Step 1: $S(\mathcal{H})$ is nonempty}

We will show that $\rho(\epsilon f ; a)$ is a homologically essential critical value.

Consider an almost complex structure $J_{0}$ such that $-\epsilon f$ is Morse-Smale for the metric $g=\omega\left(\cdot, J_{0} \cdot\right)$. We denote by $C M_{*}(-\epsilon f)$ the graded Morse complex associated to $\left(-\epsilon f, J_{0}\right)$. An element of $C M_{\ell}(-\epsilon f)$ has the form

$$
\sum_{k} a_{k}\left[p_{k}\right], \quad a_{k} \in \mathbb{Q}, p_{k} \in \mathrm{Crit}_{\ell}(-\epsilon f) .
$$

For given $p \in \mathrm{Crit}_{*}(-\epsilon f)$ and $A \in \Gamma$, we denote $p \cdot q^{A}:=[p, \widehat{p} \# A]$, where $\widehat{p}$ is the constant disc $p$.

We represent $a^{b}$ by a Novikov Morse cycle

$$
\gamma=\sum_{A} \gamma_{A} q^{A}, \quad \gamma_{j} \in C M_{*}(-\epsilon f)
$$


of $-\epsilon f$. As we argued in [Section 5, 15], we may assume that the representative $\gamma$ of $a^{b}$ has the form

$$
\gamma=\sum_{A \in \Gamma(a)} \gamma_{A} q^{A}
$$

where $\gamma_{A}$ is a Morse cycle of $-\epsilon f$. By the definition of Novikov Floer chains, we can enumerate $\Gamma(a)$ so that $\lambda_{j}=\omega\left(A_{j}\right)$ satisfy

$$
\lambda_{1}>\lambda_{2}>\cdots \text {. }
$$

Then it is easy to see

$$
\lambda_{\epsilon f}(\gamma)=\lambda_{\epsilon f}\left(\gamma_{1} q^{A_{1}}\right)
$$

provided $\epsilon>0$ satisfies

$$
\epsilon(\max f-\min f) \leq c(a)=\lambda_{1}-\lambda_{2}
$$

(See the proof of [Lemma 7.4, 15].) On the other hand, we compute

$$
\lambda_{\epsilon f}\left(\gamma_{1} q^{A_{1}}\right)=\mathcal{A}_{\epsilon f}\left(p_{\gamma_{1}} q^{A_{1}}\right)=-\omega\left(A_{1}\right)-\epsilon f\left(p_{\gamma_{1}}\right),
$$

where $p_{\gamma_{1}}$ is the peak of the Morse cycle $\gamma_{1}$ measured by the values of $-\epsilon f$. Recall that as long as we fixed the quantum cohomology class a, the collection $\Gamma(a)$ is fixed and so $A_{1}$ is fixed and the coefficient Morse cycles $\gamma_{1}$ varies inside the homology classes $P D\left(a_{1}\right) \in H_{*}(M, \mathbb{Q})$.

Therefore if we choose $\epsilon$ so that $(9.5)$ is satisfied, the mini-max value $\rho(\epsilon f ; a)$ becomes

$$
\rho(\epsilon f ; a)=-\omega\left(A_{1}\right)+\inf _{\gamma_{1} \in P D\left(a_{1}\right)} \max _{p}\left\{-\epsilon f(p) \mid p \in \gamma_{1}\right\}
$$

However it is an easy consequence of compactness of $M$ and is well-known in the finite dimensional critical point theory that on a compact manifold $M$, the mini-max value

$$
\inf _{\gamma_{1} \in P D\left(a_{j}\right)} \max _{p}\left\{-\epsilon f(p) \mid p \in \gamma_{1}\right\}
$$

can be realized by the level of a tight Morse cycle $\gamma_{t g t}$. Then if we re-choose $\gamma_{1}=\gamma_{t g t}$ and fix other $\gamma_{j}$ 's for $j \geq 2$, we have

$$
\rho(\epsilon f ; a)=\mathcal{A}_{\epsilon f}\left(\left[p_{\left(f ; a_{1}\right)}, p_{\left(f ; a_{1}\right)} q^{A_{1}}\right]\right)=\lambda_{\epsilon f}(\gamma) .
$$

Here $p_{\left(f ; a_{1}\right)}$ is the unique critical point of $f$ that is the peak of the tight Morse cycle $\gamma_{1}$ associated to the cohomology class $a_{1} \in H^{*}(M)$. This proves that $\rho(\epsilon f ; a)$ is a homologically essential critical value. 


\section{Step 2: $S(\mathcal{H})$ is open in $[0,1]$}

Let $\eta_{0} \in S(\mathcal{H})$. We would like to show that there exists $\delta>0$ such that $\left(\eta_{0}-\delta, \eta_{0}+\delta\right) \subset S(\mathcal{H})$. We consider two cases separately: one is the case where $\eta_{0} \in[0,1] \backslash \mathcal{S i n g}(\mathcal{H})$ and the other the case with $\eta_{0} \in \operatorname{Sing}(\mathcal{H})$.

For the case where $\eta_{0} \in[0,1] \backslash\left(\mathcal{S i n g}(\mathcal{H})\right.$, we choose $j \in \mathcal{P}^{s u b}\left(j_{\omega} ; \mathcal{H} ; \eta_{0}\right)$, i.e., $\eta_{0}$ is a Floer point for $(\mathcal{H}, j)$. See Section 4 for the definition of $\mathcal{P}^{s u b}\left(j_{\omega} ; \mathcal{H} ; \eta_{0}\right)$. Then the stability theorem, Theorem 8.3 implies existence of such $\delta>0$.

Next, we consider the case when $\eta_{0}$ is in $\operatorname{Sing}(\mathcal{H}) \cap S(\mathcal{H})$. In this case, by the definition of $S(\mathcal{H})$, there is $j \in \mathcal{P}\left(j_{\omega} ; \mathcal{H}\right)$ for which we have a sequence of the Floer points $\eta_{k} \rightarrow \eta_{0}$ and tight Floer cycles $\alpha_{k} \in C F_{*}\left(H\left(\eta_{k}\right)\right)$. Under this assumption, we would like to prove that there exists $\delta_{6}=\delta_{6}\left(\mathcal{H}, j ; \eta_{0}\right)>0$ such that all $\eta \in\left(\eta_{0}-\delta_{6}, \eta_{0}+\delta_{6}\right) \backslash\left\{\eta_{0}\right\}$ allows tight cycles with its level $\rho(H(\eta) ; a)$. As in Section 8, we will assume, without loss of any generality,

$$
\eta_{k} \nearrow \eta_{0}
$$

We denote $\left[z_{k}, w_{k}\right]$ be a peak of $\alpha_{k}$. Then we have

$$
\lim _{k \rightarrow \infty} \mathcal{A}_{H\left(\eta_{k}\right)}\left(\left[z_{k}, w_{k}\right]\right)=\lim _{k \rightarrow \infty} \rho\left(H\left(\eta_{k}\right) ; a\right)=\rho\left(H\left(\eta_{0}\right) ; a\right)
$$

by the continuity of $\rho_{a}$. After choosing a subsequence, we may assume that $z_{k}$ converges to a periodic orbit $z_{\infty} \in \operatorname{Per}\left(H\left(\eta_{0}\right)\right)$.

Using the Cerf property of $\mathcal{H}$, there are two cases to consider: one is the case where

$$
z_{k} \neq z^{ \pm}\left(\eta_{k}\right)
$$

and the other

$$
z_{k}=z^{+}\left(\eta_{k}\right) \quad \text { or } \quad z_{k}=z^{-}\left(\eta_{k}\right)
$$

after choosing a subsequence of $\eta_{k}$ if necessary.

Note that for the case (9.9), the limit orbit is nothing but

$$
z_{\infty}=z_{0}
$$

where $z_{0}$ is the unique degenerate periodic orbit of $H\left(\eta_{0}\right)$ and for the case (9.8), the limit $z_{\infty}$ is far away from $z_{0}$. The following proposition reduces the proof to the case of (9.8).

Proposition 9.2. Let $a \neq 0$ be a given quantum cohomology class and denote by $\alpha$ a Floer cycle with $[\alpha]=a^{b}$. Then there exists $0<\delta_{9} \leq \delta_{1}$ with $\delta_{9}=$ $\delta_{9}(\mathcal{H}, j)$ such that at any Floer point $\eta$ satisfying $d(\eta, \mathcal{S i n g}(\mathcal{H}))<\delta_{9}$ there exists a tight Floer cycle $\alpha$ of $(H(\eta), J(\eta))$, no peak of which is of the form

$$
\left[z^{ \pm}(\eta), w\right]
$$

for any bounding disc $w$.

Proof. We have already shown in Proposition 6.9 that no peak of $\alpha$ is of the form $\left[z^{+}(\eta), w\right]$. We now prove applying Theorem 6.7 (2) and tightness of $\alpha$ that there exists $\delta_{9}$ such that whenever $\left|\eta-\eta_{0}\right|<\delta_{9}$, if there is a peak of the 
tight cycle $\alpha$ having the form $\left[z^{-}(\eta), w^{-}(\eta)\right]$, we can cancel the peak by adding $\partial_{(H(\eta), J(\eta))}\left(c \cdot\left[z^{+}(\eta), w^{+}(\eta)\right]\right)$ with $w^{+}(\eta)=w^{-}(\eta) \# u_{z^{-}(\eta) z^{+}(\eta)}^{c a n}$ for a suitable $c \in \mathbb{Q}$ keeping the level unchanged and hence keeping tightness of $\alpha$ as well. Applying this cancelling repeatedly, we can cancel all such peaks. This finishes the proof.

We therefore safely assume that we are in the case of (9.8). Then an examination of the proof of Theorem 8.3 proves the following:

Lemma 9.3. There exists $\delta_{4}^{\prime}=\delta_{4}^{\prime}\left(\mathcal{H}, j ; a, \eta_{0}\right)>0$ and a sufficiently large $N \in \mathbb{N}$ such that the transferred cycles

$$
h_{\left(\eta_{N} \eta ; \rho_{0}\right)}\left(\alpha_{N, \pm}\right)
$$

are tight on each of the semi-intervals of $\left(\eta_{0}-\delta_{4}^{\prime}, \eta_{0}+\delta_{4}^{\prime}\right) \backslash\left\{\eta_{0}\right\}$.

Proof. The proof is a variation of that of Proposition 8.4 except that in the current case, $\eta_{0} \in \operatorname{Sing}(\mathcal{H})$ and so the Floer homology itself at $\eta_{0}$ is not defined. On the other hand, by the Cerf property of $\mathcal{H}$, there is no critical point $[z, w]$ of $\mathcal{A}_{H\left(\eta_{0}\right)}$ at the same level of the form $\mathcal{A}_{H\left(\eta_{0}\right)}\left(\left[z_{0}, w_{0}\right]\right)$, where $z_{0}$ is a degenerate periodic orbit.

Consider the constants $\lambda_{1}$ and $\lambda_{2}$ that appears in the proof of Theorem 8.3. An examination of the proof shows that we can choose $\lambda_{1}$ depending only on $(\mathcal{H}, j)$ but independent of $\eta_{0}$ if we replace $A_{\left(H\left(\eta_{0}\right), J\left(\eta_{0}\right)\right)}$ by the constant $A_{(\mathcal{H}, j)}$ provided in Theorem 6.7.

For the constant $\lambda_{2}=\lambda_{2, K}$, we take $\lambda_{2, K}$ given in Lemma 8.10 for

$$
K=3 \delta+c,
$$

where $\delta, c$ are as in the proof of Theorem 8.3. Once we have this, the same argument as that of Theorem 8.3 with $\lambda_{2}\left(\eta_{0}\right)$ replaced by $\lambda_{2, K}$ provides the constant $\delta_{4}^{\prime}=\delta_{4}^{\prime}\left(\mathcal{H}, j ; a, \eta_{0}\right)>0$ which finishes the proof.

If we choose $N$ sufficiently large, then $\eta_{0} \in\left(\delta_{4}^{\prime}-\eta_{N}, \delta_{4}^{\prime}+\eta_{N}\right)$ and hence follows openness of $S(\mathcal{H})$ at $\eta_{0}$. Combining the above altogether, we have finished the proof of openness of $S(\mathcal{H})$.

We remark that the corresponding level functions

$$
\begin{aligned}
& \mu_{-}(\eta)=\lambda_{H(\eta)}\left(h_{\eta_{0} \eta}\left(\alpha_{N,-}\right)\right) \\
& \mu_{+}(\eta)=\lambda_{H(\eta)}\left(h_{\eta_{0} \eta}\left(\alpha_{N,+}\right)\right)
\end{aligned}
$$

together define a continuous function on $\left(\eta_{N}-\delta_{4}^{\prime}, \eta_{N}+\delta_{4}^{\prime}\right)$ which extends continuously across $\eta_{0}$.

\section{Step 3: $S(\mathcal{H})$ is closed in $[0,1]$}

Let $\eta_{k} \rightarrow \eta_{\infty}$ be a sequence of Floer points such that each $\rho\left(H\left(\eta_{i}\right) ; a\right)$ is a homologically essential critical value. First consider the case where $\eta_{\infty}$ lies in 
$[0,1] \backslash \operatorname{Sing}(\mathcal{H})$. We choose $j \in \mathcal{P}^{s u b}\left(j_{\omega} ; \mathcal{H} ; \eta_{\infty}\right)$ and consider the transferred cycles

$$
\alpha_{k}(\eta):=h_{\left(\eta_{k} \eta ; \rho_{0}\right)}\left(\alpha_{k}\right) .
$$

Proposition 8.2 shows that the existence of tight cycles at $\eta_{k}$ does not depend on the choice of $j$ and so we may assume that all $\eta_{k}$ including $\eta_{\infty}$ are Floer points of $(\mathcal{H}, j)$. Then by the same arguments used for the proof of Theorem 8.3 and using Lemma 8.10, there is a constant $\delta_{4}=\delta_{4}\left(\eta_{\infty}\right)>0$ such that on each of the semi-intervals of $\left(\eta_{k}-\delta_{4}, \eta_{k}\right]$ or $\left[\eta_{k}, \eta_{k}+\delta_{4}\right)$, we can find a tight cycle $\alpha_{k}$ for which $\alpha_{k}(\eta)$ is also tight on the corresponding intervals. Obviously if $k$ is sufficiently large, then $\eta_{k} \in\left(\eta_{\infty}-\delta, \eta_{\infty}+\delta\right)$. In particular, $h_{\left(\eta_{k} \eta_{\infty} ; \rho_{0}\right)}\left(\alpha_{k}\right)$ is a required tight Floer cycle of $H\left(\eta_{\infty}\right)$ at the level $\rho\left(H\left(\eta_{\infty}\right) ; a\right)$. This takes care of the case when $\eta_{\infty}$ lies in $[0,1] \backslash \operatorname{Sing}(\mathcal{H})$.

On the other hand when $\eta_{\infty} \in S(\mathcal{H}) \cap \operatorname{Sing}(\mathcal{H})$, there is nothing to prove by the definition of $S(\mathcal{H})$. This proves that $S(\mathcal{H})$ is closed.

\section{Step 4: Finish-up of the proof}

Combining Step 1-3 and the fact that $[0,1]$ is connected, we have proved $S(\mathcal{H})=[0,1]$ and so the proof of Theorem 9.1. This in turn finishes the proof of Theorem II at last.

Remark 9.4. In fact, an examination of the above proof seems to show that the spectrality axiom holds for the Hamiltonians either of the Bott-Morse types or of a finite type: We call a Hamiltonian $H$ a finite type, if all of its periodic orbits are isolated and the degeneracy of the linearization is of finite order. It would be interesting to see if the spectrality axiom holds for arbitrary Hamiltonians or not.

We are now ready to prove Theorem $\mathrm{V}$ stated in the introduction as a byproduct of the arguments used in the above proof. We re-state the theorem here.

Theorem 9.5. Let $\mathcal{H}$ be a Cerf-homotopy. Then the spectral function $s \mapsto$ $\rho\left(H^{s} ; a\right)$ is smooth away from a countable subset of $\mathcal{C}^{\text {nd }} \operatorname{ross}(\mathcal{H})$.

Proof. Let $\eta_{0} \in(0,1) \backslash \mathcal{S i n g}(\mathcal{H})$. We choose a generic $J^{\eta_{0}}$ such that the pair $\left(H\left(\eta_{0}\right), J^{\eta_{0}}\right)$ is Floer-regular and then extend $J^{\eta_{0}}$ to a family $j=\left\{J^{\eta}\right\}_{0 \leq \eta \leq 1}$ so that the pair $(\mathcal{H}, j)$ is a Floer homotopy, i.e., satisfies the properties of Theorem 4.6. Then the above proof shows that if $\eta_{0}$ is not in $\mathcal{C}^{n d} \operatorname{ross}(\mathcal{H})$, there exists a tight Floer cycle $\alpha_{0}$ for $\left(H\left(\eta_{0}\right), J^{\eta_{0}}\right)$ such that the function $\mu$

$$
\mu(\eta)=\lambda_{H(\eta)}\left(h_{\eta_{0} \eta}\left(\alpha_{0}\right)\right)
$$

is well-defined on $\left(\eta_{0}-\delta, \eta_{0}+\delta\right)$ for some $\delta>0$ which is smooth thereon. Furthermore by making $\delta>0$ smaller if necessary, the proof of Theorem 8.3 shows that the cycles $h_{\eta_{0} \eta}\left(\alpha_{0}\right)$ are all tight and hence $\rho(H(\eta) ; a)=\mu(\eta)$. On the other hand if $\eta_{0} \in \mathcal{C}^{n d} \operatorname{ross}(\mathcal{H})$, the last paragraph of the proof of Theorem 
8.3 shows that $\rho(H(\eta) ; a)$ may be realized by two different branches $\mu_{-}:\left(\eta_{0}-\right.$ $\left.\delta, \eta_{0}\right] \rightarrow \mathbb{R}$ and $\mu_{+}:\left[\eta_{0}, \eta_{0}+\delta\right) \rightarrow \mathbb{R}$ with $\mu_{-}\left(\eta_{0}\right)=\mu_{+}\left(\eta_{0}\right)$ given by

$$
\begin{aligned}
& \mu_{-}(\eta)=\lambda_{H(\eta)}\left(h_{\eta_{0} \eta}\left(\alpha_{-}\right)\right) \\
& \mu_{+}(\eta)=\lambda_{H(\eta)}\left(h_{\eta_{0} \eta}\left(\alpha_{+}\right)\right),
\end{aligned}
$$

where $\alpha_{ \pm}$are two different tight Floer cycles of $H\left(\eta_{0}\right)$. This proves that the function $\eta \mapsto \rho(H(\eta) ; a)$ is differentiable possibly except at such points from $\mathcal{C}^{n d} \operatorname{ross}(\mathcal{H})$. This finishes the proof.

\section{§10. Spectral invariants of Hamiltonian diffeomorphisms}

We recall that the invariants $\rho(H ; a)$ were constructed for arbitrary Hamiltonian functions $H: S^{1} \times M \rightarrow \mathbb{R}$ in [18]. We first summarize the basic properties of the invariants $\rho=\rho(H ; a)$. Except the nondegenerate spectrality axiom proven in the present paper, all other axioms are proved in [18].

Theorem 10.1. Let $(M, \omega)$ be arbitrary closed symplectic manifold. For any given quantum cohomology class $0 \neq a \in Q H^{*}(M)$, we have a continuous function denoted by

$$
\rho=\rho(H ; a): C_{m}^{\infty}\left(S^{1} \times M\right) \times Q H^{*}(M) \rightarrow \mathbb{R}
$$

such that they satisfy the following axioms: Let $H, F \in C_{m}^{\infty}\left(S^{1} \times M\right)$ be smooth Hamiltonian functions and $a \neq 0 \in Q H^{*}(M)$. Then $\rho$ satisfies the following axioms:

(1) (Nondegenerate spectrality) For each $a \in Q H^{*}(M), \rho(H ; a) \in$ $\operatorname{Spec}(H)$ if $H$ is nondegenerate.

(2) (Projective invariance) $\rho(H ; \lambda a)=\rho(H ; a)$ for any $0 \neq \lambda \in \mathbb{Q}$.

(3) (Normalization) For $a=\sum_{A \in \Gamma} a_{A} q^{-A}$, we have $\rho(\underline{0} ; a)=v(a)$, where $\underline{0}$ is the zero function and

$$
v(a):=\min \left\{\omega(-A) \mid a_{A} \neq 0\right\}=-\max \left\{\omega(A) \mid a_{A} \neq 0\right\} .
$$

is the (upward) valuation of $a$.

(4) (Symplectic invariance) $\rho\left(\eta^{*} H ; \eta^{*} a\right)=\rho(H ; a)$ for any symplectic diffeomorphism $\eta$.

(5) (Triangle inequality) $\rho(H \# F ; a \cdot b) \leq \rho(H ; a)+\rho(F ; b)$.

(6) (C $C^{0}$-continuity) $|\rho(H ; a)-\rho(F ; a)| \leq\|H \# \bar{F}\|=\|H-F\|$, where $\|\cdot\|$ is the Hofer's pseudo-norm on $C_{m}^{\infty}\left(S^{1} \times M\right)$. In particular, the function $\rho_{a}: H \mapsto \rho(H ; a)$ is $C^{0}$-continuous.

(In the symplectic invariance axiom, $\eta^{*} a$ denotes the canonical pull-back of $a$ under the symplectic diffeomorphism $\phi$. In general $\eta^{*} a \neq a$ unless $\phi i s$ symplectically isotopic to the identity. In [18], the symplectic invariance was stated incorrectly as $\rho\left(\eta^{*} H ; a\right)=\rho(H ; a)$. We thank Polterovich for pointing out this error.) 
By the one-one correspondence between (normalized) $H$ and its associated Hamiltonian path $\phi_{H}: t \mapsto \phi_{H}^{t}$, one can regard the spectral function

$$
\rho_{a}: \mathcal{H}_{m}(M)=C_{m}^{\infty}([0,1] \times M) \rightarrow \mathbb{R}
$$

as a function defined on $\mathcal{P}(\operatorname{Ham}(M, \omega) ; i d)$, i.e.,

$$
\rho_{a}: \mathcal{P}(\operatorname{Ham}(M, \omega), i d) \rightarrow \mathbb{R}
$$

as described in [18]. Here we denote by $\mathcal{P}(\operatorname{Ham}(M, \omega), i d)$ the set of smooth Hamiltonian paths in $\operatorname{Ham}(M, \omega)$ and by $\widetilde{\operatorname{Ham}}(M, \omega)$ the set of path homotopy classes on $\mathcal{P}(\operatorname{Ham}(M, \omega), i d)$, i.e., the (étale) universal covering space of $\operatorname{Ham}(M, \omega)$. We equip $\widetilde{\operatorname{Ham}}(M, \omega)$ with the quotient topology. The following corollary shows that the function $\rho_{a}$ pushes down to $\widetilde{\operatorname{Ham}}(M, \omega)$ as a continuous function. The proof of the following fact is entirely the same as in the rational case [18] summarized in the introduction of the present paper, and so omitted.

Corollary 10.2. Let $(M, \omega)$ be an arbitrary closed symplectic manifold. For any nondegenerate $\widetilde{\phi} \in \widetilde{\operatorname{Ham}}(M, \omega)$, we have

$$
\rho(H ; a)=\rho(K ; a)
$$

for any smooth functions $H \sim K$ satisfying $[H]=[K]=\widetilde{\phi}$.

We like to emphasize that at this moment, because we do not know validity of the spectrality axiom for degenerate Hamiltonians, we do not have the equality

$$
\rho(H ; a)=\rho(K ; a)
$$

yet for two $H$ and $K$ representing the same $\widetilde{\phi}$, if the latter is degenerate. The scheme of the proof used to prove (1.3) for the rational $(M, \omega)$ cannot be applied without the spectrality axiom. In this regard, the following is still a non-trivial theorem to prove. The argument used in the proof is similar to the proof of [15, Lemma 5.1] or [18, Theorem 5.1].

Theorem 10.3. For any pair $(H, K)$ satisfying $H \sim K$, we have

$$
\rho(H ; a)=\rho(K ; a) .
$$

Proof. For the nondegenerate case, Corollary 10.2 proves (10.3). It remains to prove (10.3) for the degenerate cases.

Suppose $H \sim K$. We approximate $H$ and $K$ by sequences of nondegenerate Hamiltonians $H_{i}$ and $K_{i}$ in the $C^{\infty}$ topology respectively. We note that the Hamiltonian

$$
K \# H_{i} \# \bar{K}
$$

generates the flow $\phi_{K}^{t} \circ \phi_{H_{i}}^{t} \circ\left(\phi_{K}^{t}\right)^{-1}$, which is conjugate to the flow $\phi_{H_{i}}^{t}$ and is nondegenerate. Therefore we have

$$
\rho\left(H_{i} ; a\right)=\rho\left(K \# H_{i} \# \bar{K} ; a\right)
$$


by the symplectic invariance of $\rho$. On the other hand, since $H \sim K$, we have

$$
K \# H_{i} \# \bar{K} \sim K \# H_{i} \# \bar{H} .
$$

Since both are nondegenerate, Corollary 10.2 implies

$$
\rho\left(K \# H_{i} \# \bar{K} ; a\right)=\rho\left(K \# H_{i} \# \bar{H} ; a\right) .
$$

Here we remind the readers that the definition of the nondegeneracy of a Hamiltonian depends only on its time-one map. By taking the limits of (10.4) and (10.5) and using the continuity of $\rho(\cdot ; a)$, we get

$$
\rho(H ; a)=\rho(K \# H \# \bar{K} ; a)=\rho(K \# H \# \bar{H} ; a)=\rho(K ; a),
$$

where the last equality comes since $H \# \bar{H}=0$. Hence the proof.

Therefore, for any nondegenerate $\widetilde{\phi}$, we can define the value $\rho_{a}(\widetilde{\phi})$ by

$$
\rho_{a}(\widetilde{\phi}):=\rho(H ; a)
$$

for $H$ satisfying $[H]=\widetilde{\phi}$. This defines a well-defined continuous function

$$
\rho_{a}: \widetilde{\operatorname{Ham}}^{n d}(M, \omega) \rightarrow \mathbb{R}
$$

where $\widetilde{\operatorname{Ham}}^{n d}(M, \omega)$ is the subset of $\widetilde{\operatorname{Ham}}(M, \omega)$ consisting of nondegenerate $\widetilde{\phi}$ 's.

Theorem 10.4. The function $\rho_{a}$ defined by (10.7) extends to continuously $\widetilde{\operatorname{Ham}}(M, \omega)$ in the quotient topology of $\widetilde{\operatorname{Ham}}(M, \omega)$ induced from

$$
\mathcal{P}(\operatorname{Ham}(M, \omega), i d) .
$$

Proof. Recall the definition of the quotient topology under the projection

$$
\pi: \mathcal{P}(\operatorname{Ham}(M, \omega), i d) \rightarrow \widetilde{\operatorname{Ham}}(M, \omega) .
$$

We proved that the assignment

$$
H \mapsto \rho(H ; a)
$$

is continuous on $C^{\infty}([0,1] \times M)$ in [18]. By the definition of the quotient topology,

$$
\rho_{a}: \widetilde{\operatorname{Ham}}(M, \omega) \rightarrow \mathbb{R}
$$

is continuous, because the composition

$$
\rho_{a} \circ \pi: \mathcal{P}(\operatorname{Ham}(M, \omega), i d) \rightarrow \mathbb{R},
$$

which is nothing but (10.8), is continuous. 
Remark 10.5. One cheap way of defining a function on the covering space $\widetilde{\operatorname{Ham}}(M, \omega)$ out of the spectral invariants $\rho(H ; a)$ is to take the infimum of $\rho(H ; a)$ among all $H$ 's connecting the identity to the given time one map $\phi=$ $\phi_{H}^{1}$ in the same homotopy class of paths: i.e.,

$$
\rho^{\prime}(\widetilde{\phi} ; a):=\inf _{\widetilde{\phi}=[H]} \rho(H ; a)
$$

similarly as in the cases of Hofer pseudo-norm

$$
\|\widetilde{\phi}\|=\inf _{\widetilde{\phi}=[H]}\|H\| .
$$

However, unless we had the spectrality axiom, more specifically without its consequence Theorem 10.3, there would be no reason why the function $\rho^{\prime}$ : $\widetilde{\operatorname{Ham}}(M, \omega) \rightarrow \mathbb{R}$ is continuous just as the function $\widetilde{\phi} \mapsto\|\widetilde{\phi}\|$ is not a priori continuous in the (étale) topology of $\widetilde{\operatorname{Ham}}(M, \omega)$.

Finally, for the reader's convenience, we summarize the basic axioms of the invariant $\rho: \widetilde{\operatorname{Ham}}(M, \omega) \times Q H^{*}(M) \rightarrow \mathbb{R}$ in the following theorem, whose proofs immediately follow from Theorem 10.1 and 9.4 .

Theorem 10.6. Let $(M, \omega)$ be any closed symplectic manifold. Let $\widetilde{\phi}, \widetilde{\psi} \in$ $\widetilde{\operatorname{Ham}}(M, \omega)$ and $0 \neq a \in Q H^{*}(M)$. Then for each $0 \neq a \in Q H^{*}(M)$, the function

$$
\rho_{a}: \widetilde{\operatorname{Ham}}(M, \omega) \rightarrow \mathbb{R}
$$

is continuous, and the function

$$
\rho: \widetilde{\operatorname{Ham}}(M, \omega) \times Q H^{*}(M) \rightarrow \mathbb{R}
$$

satisfies the following axioms:

(1) (Nondegenerate spectrality) For each $a \in Q H^{*}(M), \rho(\widetilde{\phi} ; a) \in$ $\operatorname{Spec}(\widetilde{\phi})$, if $\widetilde{\phi}$ is nondegenerate.

(2) (Projective invariance) $\rho(\widetilde{\phi} ; \lambda a)=\rho(\widetilde{\phi} ; a)$ for any $0 \neq \lambda \in \mathbb{Q}$.

(3) (Normalization) For $a=\sum_{A \in \Gamma} a_{A} q^{-A}$, we have $\rho(\underline{0} ; a)=v(a)$, where $\underline{0}$ is the identity in $\widehat{\operatorname{Ham}}(M, \omega)$ and

$$
v(a):=\min _{A}\left\{\omega(-A) \mid a_{A} \neq 0\right\}=-\max \left\{\omega(A) \mid a_{A} \neq 0\right\}
$$

is the (upward) valuation of a.

(4) (Symplectic invariance) $\rho\left(\eta^{-1} \widetilde{\phi} \eta ; \eta^{*} a\right)=\rho(\widetilde{\phi} ; a)$ for any symplectic diffeomorphism $\eta$.

(5) (Triangle inequality) $\rho(\widetilde{\phi} \cdot \widetilde{\psi} ; a \cdot b) \leq \rho(\widetilde{\phi} ; a)+\rho(\widetilde{\psi} ; b)$.

(6) ( $\boldsymbol{C}^{\mathbf{0}}$-continuity) $|\rho(\widetilde{\phi} ; a)-\rho(\widetilde{\psi} ; a)| \leq\left\|\widetilde{\phi} \circ \widetilde{\psi}^{-1}\right\|$, where $\|\cdot\|$ is the Hofer's pseudo-norm on $\widehat{\operatorname{Ham}}(M, \omega)$. In particular, the function $\rho_{a}$ : $\widetilde{\phi} \mapsto \rho(\widetilde{\phi} ; a)$ is $C^{0}$-continuous. 
We like to remind the readers the spectrality axiom holds for any $\widetilde{\phi}$ for the rational symplectic manifolds [18]. It is an interesting problem to investigate to understand what kind of Hamiltonians, besides nondegenerate ones, satisfy the spectrality axiom, which will be a subject of future study.

\section{$\S 11$. Applications to Hofer's geometry}

In this section, we provide two immediate applications of the spectrality axiom in the study of Hofer's geometry of the Hamiltonian diffeomorphism group. We first recall the following definitions

$$
\begin{aligned}
& E^{-}(\widetilde{\phi})=\inf _{[\phi, H]=\widetilde{\phi}} \int_{0}^{1}-\min H_{t} d t \\
& E^{+}(\widetilde{\phi})=\inf _{[\phi, H]=\widetilde{\phi}} \int_{0}^{1} \max H_{t} d t
\end{aligned}
$$

(See [20], and [12] for example). Note that we have

$$
E^{-}(\bar{H})=E^{+}(H)
$$

for the Hamiltonian $\bar{H} \mapsto \phi^{-1}$. This in turn implies

$$
E^{-}\left(\widetilde{\phi}^{-1}\right)=E^{+}(\widetilde{\phi})
$$

and hence

$$
0 \leq E^{+}(\widetilde{\phi})+E^{-}(\widetilde{\phi}) \leq \inf _{[\phi, H]=\widetilde{\phi}} \int\left(\max H_{t}-\min H_{t}\right) d t .
$$

In particular we have the inequality for the Hofer pseudo-norm $\|\phi\|$ and $\|\phi\|_{\text {med }}$

$$
\|\phi\|_{\text {med }}:=\inf _{\pi(\widetilde{\phi})=\phi}\left(E^{+}(\widetilde{\phi})+E^{-}(\widetilde{\phi})\right) \leq\|\phi\| .
$$

Now we consider the invariant $\rho(H ; 1)$. We have shown, by definition,

$$
\rho(\widetilde{\phi} ; 1)=\rho(H ; 1)
$$

for any $H$ with $\widetilde{\phi}=[H]$. Since we have

$$
\rho(H ; 1) \leq E^{-}(H),
$$

(11.4) implies

$$
\rho(\widetilde{\phi} ; 1) \leq E^{-}(H)
$$

for all $H$ with $[H]=\widetilde{\phi}$. By taking the infimum of (11.5) over all such $H$, we have proved the following inequality

$$
\rho(\widetilde{\phi} ; 1) \leq E^{-}(\widetilde{\phi}) .
$$

Applying the same argument to $\bar{H}$ and using $E^{-}(\bar{H})=E^{+}(H)$, we obtain

$$
\rho\left(\widetilde{\phi}^{-1} ; 1\right) \leq E^{+}(H) \text {. }
$$


We now define

$$
\widetilde{\gamma}(\widetilde{\phi})=\rho(\widetilde{\phi} ; 1)+\rho\left(\widetilde{\phi}^{-1} ; 1\right)
$$

Furthermore we have

$$
\widetilde{\gamma}(\widetilde{\phi})=\rho(\widetilde{\phi} ; 1)+\rho\left(\widetilde{\phi}^{-1} ; 1\right) \geq \rho(i d ; 1)=0 .
$$

We recall the definition of the spectral norm $\gamma$ from [19]

$$
\gamma(\phi)=\inf _{H \mapsto \phi}(\rho(H ; 1)+\rho(\bar{H} ; 1))=\inf _{\pi(\widetilde{\phi})=\phi}(\widetilde{\gamma}(\widetilde{\phi})) .
$$

Combining (11.5) and (11.7), we have proved:

Theorem 11.1. For any $\widetilde{\phi} \in \widetilde{\operatorname{Ham}}(M, \omega)$, we have

$$
\widetilde{\gamma}(\widetilde{\phi}) \leq\|\widetilde{\phi}\| \text {. }
$$

In particular, we have

$$
\gamma(\phi) \leq\|\phi\|_{\text {med }}
$$

(11.9) is an improvement of the inequality $\gamma(\phi) \leq\|\phi\|$ proven in [19] and give a different proof of nondegeneracy, with a lower bound, of the medium Hofer norm $\|\cdot\|_{\text {med }}$. Previously McDuff [12] proved that this is nondegenerate by a different method.

Next we define a partial order on $\widetilde{\operatorname{Ham}}(M, \omega)$ out of the invariant $\rho(\widetilde{\phi} ; 1)$. We note that $\rho(\widetilde{\phi} ; 1)$ can be strictly negative although the sum $\rho(\widetilde{\phi} ; 1)+\rho\left(\widetilde{\phi}^{-1} ; 1\right)$ cannot. We recall our convention of the action functional is

$$
\mathcal{A}_{H}([z, w])=-\int w^{*} \omega-\int H(t, z(t)) d t
$$

emphasizing the ' - ' in front of the integral $\int H(t, z(t)) d t$, and that $\rho(H ; a)$ is defined in terms of the action functional, not in terms of $H$ itself. This explains somewhat contradictory usage of positiveness in the definition.

Definition 11.2. We say that a Hamiltonian $H$ is homologically positive if

$$
\phi(H ; 1) \leq 0,
$$

and $\widetilde{\phi} \in \widetilde{\operatorname{Ham}}(M, \omega)$ is homologically positive if its representing normalized Hamiltonian $H$ is homologically positive. We also call the corresponding Hamiltonian path $\left\{\phi_{H}^{t}\right\}_{0 \leq t \leq 1}$ homologically positive if $[\phi, H]=\widetilde{\phi}$ is positive. We define

$$
\begin{aligned}
& \widetilde{\operatorname{Ham}}_{+}(M, \omega)=\{\widetilde{\phi} \mid \widetilde{\phi} \text { homologically positive }\} \\
& C_{m}^{+}([0,1] \times M)=\left\{H \in C_{m}^{\infty}\left(S^{1} \times M\right) \mid H \text { homologically positive }\right\}
\end{aligned}
$$

and denote

$$
\begin{gathered}
\mathcal{P}^{+}(\operatorname{Ham}(M, \omega), i d)=\{f:[0,1] \rightarrow \operatorname{Ham}(M, \omega) \mid f(0)=i d, \\
\left.f(t)=\phi_{H}^{t}, H \in C_{m}^{+}\left(S^{1} \times M\right)\right\}
\end{gathered}
$$


for the set of homologically positive Hamiltonian paths issued at the identity.

We refer readers to [3] for a general discussion on partially ordered groups and the definition of the normal cone used below in the context of contact transformations.

Theorem 11.3. The subset $C:=\widetilde{\operatorname{Ham}}_{+}(M, \omega)$ forms a normal cone in $\mathcal{D}:=$ $\widetilde{\operatorname{Ham}}(M, \omega)$, i.e., $C$ satisfies

(1) If $f, g \in C, f g \in C$,

(2) If $f \in C$ and $h \in \mathcal{D}$, $h f h^{-1} \in C$,

(3) $i d \in C$.

Proof. (1) follows from the triangle inequality

$$
\rho(f ; 1)+\rho(g ; 1) \geq \rho(f g ; 1)
$$

and (2) from the symplectic invariance of $\rho$ and $(3)$ from the identity $\rho(\underline{0} ; 1)=$ 0 .

Following [3], we define the partial order associated to this normal cone on $\widehat{\operatorname{Ham}}(M, \omega)$ by

$$
f \geq g \text { on } \mathcal{D} \text { if and only if } f g^{-1} \in C .
$$

The question whether this is non-trivial, i.e., satisfies the axiom

$$
f \leq g \& g \leq f \text { if and only if } f=g
$$

is an interesting problem to study and is related to the study of Hamiltonian loops $h$ and the corresponding spectral invariants $\rho(h ; 1)$. This is a subject of future study. Viterbo [23] had earlier introduced the notion of positive Hamiltonians and a similar partial order for the set of compactly supported Hamiltonians on $\mathbb{R}^{2 n}$ and proved nondegeneracy of the partial order.

\section{Appendix}

\section{A.1. Proof of Proposition 2.7}

We first recall the definition from [2], [21] of the Conley-Zehnder index for a path $\alpha$ lying in $\mathcal{S} P^{*}(1)$ where we denote

$$
\mathcal{S} P^{*}(1)=\{\alpha:[0,1] \rightarrow S p(2 n, \mathbb{R}) \mid \alpha(0)=i d, \operatorname{det}(\alpha(1)-i d) \neq 0\}
$$

following the notation from [21]. We denote by $\mu_{C Z}(\alpha)$ the Conley-Zehnder index of $\alpha$ given in [21].

Next we note that a given pair $[\gamma, w] \in \widetilde{\Omega}_{0}(M)$ determines a preferred homotopy class of trivialization of the symplectic vector bundle $\gamma^{*} T M$ on $S^{1}=\partial D^{2}$ that extends to a trivialization

$$
\Phi_{w}: w^{*} T M \rightarrow D^{2} \times\left(\mathbb{R}^{2 n}, \omega_{0}\right)
$$

over $D^{2}$ of where $D^{2} \subset \mathbb{C}$ is the unit disc with the standard orientation. Any one-periodic solution $z: \mathbb{R} / \mathbb{Z} \rightarrow M$ of $\dot{x}=X_{H}(x)$ has the form $z(t)=\phi_{H}^{t}(p)$ 
for a fixed point $p=z(0) \in \operatorname{Fix}\left(\phi_{H}^{1}\right)$. For the given one-periodic solution $z$ and its bounding disc $w: D^{2} \rightarrow M$, we consider the one-parameter family of the symplectic maps

$$
d \phi_{H}^{t}(z(0)): T_{z(0)} M \rightarrow T_{z(t)} M
$$

and define a map $\alpha_{[z, w]}:[0,1] \rightarrow S p(2 n, \mathbb{R})$ by

$$
\alpha_{[z, w]}(t)=\Phi_{w}(z(t)) \circ d \phi_{H}^{t}(z(0)) \circ \Phi_{w}(z(0))^{-1} .
$$

Obviously we have $\alpha_{[z, w]}(0)=i d$, and nondegeneracy of $H$ implies that

$$
\operatorname{det}\left(\alpha_{[z, w]}(1)-i d\right) \neq 0
$$

and hence

$$
\alpha_{[z, w]} \in \mathcal{S} P^{*}(1)
$$

In general, according to the definition from [2] of the Conley-Zehnder index for a paths $\alpha$ lying in $\mathcal{S} P^{*}(1)$, the Conley-Zehnder index of $[z, w]$ is defined by

$$
\mu_{H}([z, w]):=\mu_{C Z}\left(\alpha_{[z, w]}\right) .
$$

Now we start with the proof of Proposition 2.6. We can write

$$
z^{\prime}(t)=\exp _{z(t)} \xi(t), \quad \xi(t) \in T_{z(t)} M
$$

since $z, z^{\prime}$ is an associated pair of $H, H^{\prime}$ that are assumed to be sufficiently $C^{2}$ close. We denote by

$$
\Pi_{0, t}^{s, t}: T_{z(t)} M \rightarrow T_{z^{s}(t)} M
$$

the parallel translation along the short geodesics $r \mapsto \exp _{z(t)}(r \xi(t))$ for $0 \leq r \leq$ $s$ where $z^{s}: S^{1} \rightarrow M$ are the loops defined by

$$
z^{s}(t)=\exp _{z(t)}(s \xi(t)), \quad s \in[0,1] .
$$

Now we make an identification

$$
\Pi: u^{*} T M \rightarrow[0,1] \times z^{*} T M
$$

for $u=u_{z z^{\prime}}^{c a n}$ by the map

$$
v \in\left(u^{*} T M\right)_{(s, t)} \mapsto\left(s,\left(\Pi_{0, t}^{s, t}\right)^{-1}(v)\right) \in\{s\} \times\left(z^{*} T M\right)_{t} .
$$

Then noting that we can write

$$
\left(w^{\prime}\right)^{*} T M=w^{*} T M \# u^{*} T M
$$

for $w^{\prime}=w \# u, \Phi_{w}$ and $\Pi$ together induce a natural trivialization

$$
\Phi_{w^{\prime}}:\left(w^{\prime}\right)^{*} T M \rightarrow\left(D^{2} \cup[0,1] \times S^{1}\right) \times \mathbb{R}^{2 n}
$$

by the formula

$$
\Phi_{w^{\prime}}(v)= \begin{cases}\Phi_{w}(\xi) & \xi \in w^{*} T M \\ \left(\left.\Phi_{w}\right|_{\partial D^{2}}\right)\left(\Phi_{u}(\xi)\right) & \xi \in u^{*} T M\end{cases}
$$


By the nondegeneracy hypothesis of $z$, we know that the map $\alpha_{[z, w]}:[0,1] \rightarrow$ $S p(2 n, \mathbb{R})$ defines a path in $\mathcal{S} P^{*}(1)$. We compare the two linearized vector fields of $X_{H}$ and $X_{H^{\prime}}$ along the corresponding periodic orbits $z$ and $z^{\prime}$

$$
\left(\left.\Phi_{w}\right|_{\partial D}\right)_{*}\left(D X_{H}(z)\right)
$$

and

$$
\left(\left.\Phi_{w^{\prime}}\right|_{\partial D}\right)_{*}\left(D X_{H^{\prime}}\left(z^{\prime}\right)\right)
$$

respectively. We can express

$$
\begin{aligned}
\left(\left.\Phi_{w^{\prime}}\right|_{\partial D}\right)_{*}\left(D X_{H^{\prime}}\left(z^{\prime}\right)\right) & =\left(\left.\Phi_{w}\right|_{\partial D^{2}}\right) \circ\left(\Pi_{0}^{1}\right)^{-1} \circ\left(D X_{H^{\prime}}\left(z^{\prime}\right)\right) \circ \Pi_{0}^{1} \circ\left(\left.\Phi_{w}\right|_{\partial D^{2}}\right)^{-1} \\
& =\left(\left.\Phi_{w}\right|_{\partial D^{2}}\right) \circ\left(\left(\Pi_{0}^{1}\right)^{-1} \circ D X_{H^{\prime}}\left(z^{\prime}\right) \circ \Pi_{0}^{1}\right) \circ\left(\left.\Phi_{w}\right|_{\partial D^{2}}\right)^{-1} .
\end{aligned}
$$

Since we assume that $H^{\prime}$ is $C^{2}$ close to $H$ and $\left(z, z^{\prime}\right)$ is an associated pair, it can be easily seen

$$
\left(\Pi_{0}^{1}\right)^{-1} \circ D X_{H^{\prime}}\left(z^{\prime}\right) \circ \Pi_{0}^{1}: z^{*} T M \rightarrow z^{*} T M
$$

is $C^{1}$ close to $D X_{H}(z): z^{*} T M \rightarrow z^{*} T M$. Therefore if we write the flow of the linearization

$$
\Phi_{w^{\prime}}\left(z^{\prime}(t)\right) \circ d \phi_{H^{\prime}}^{t}\left(z^{\prime}(0)\right) \circ \Phi_{w^{\prime}}\left(z^{\prime}(0)\right)^{-1}
$$

of $\left(\Phi_{w^{\prime}}\right)_{*}\left(D X_{H^{\prime}}\left(z^{\prime}\right)\right)(1, t, \xi)$ as

$$
\left(1, t, \alpha_{[z, w]}^{\prime}(t) \xi\right)
$$

the map $\alpha_{\left[z, w^{\prime}\right]}^{\prime}:[0,1] \rightarrow S p(2 n, \mathbb{R})$ is $C^{1}$ close to $\alpha_{[z, w]}$. Therefore we can homotope $\alpha_{\left[z^{\prime}, w^{\prime}\right]}^{\prime}$ to $\alpha_{[z, w]}$ inside $\mathcal{S} P^{*}(1)$. Then by an invariance property of the Conley-Zehnder index [21] under such a homotopy, we obtain

$$
\mu\left(\alpha_{[z, w]}\right)=\mu\left(\alpha_{\left[z^{\prime}, w^{\prime}\right]}^{\prime}\right) .
$$

By the definition of the Conley-Zehnder index $\mu_{H}([z, w])$, this implies $\mu_{H}[z, w]$ $=\mu_{H^{\prime}}\left(\left[z^{\prime}, w^{\prime}\right]\right)$ which finishes the proof.

\section{A.2. Proof of Lemma 6.8}

We first prove the following general lemma. The lemma is stated in a more general form than needed for the purpose of using it also in the proof of Proposition 9.2 in Section 9. The present case corresponds to the case of

$$
\left(H_{\alpha}, J_{\alpha}\right)=\left(H_{\beta}, J_{\beta}\right)=(H, J) \equiv(\mathcal{H}, j) .
$$

Lemma A.1. Let $(H, J)$ be an pair consisting of smooth one-periodic families of Hamiltonians and almost complex structures. Denote $\left(J_{\alpha}, H_{\alpha}\right)$ and $\left(J_{\beta}, H_{\beta}\right)$ be any regular pairs in the Floer theoretic sense for which the Floer homologies are defined. Let $(\mathcal{H}, j)$ be a homotopy pair with $\mathcal{H}$ connecting $H_{\alpha}, H_{\beta}$ for which the Floer chain map $h_{\left(j, \mathcal{H}^{\prime} ; \rho\right)}$ is defined for a cut-off function. Let $U$ be any fixed tubular neighborhood of $z$ with smooth boundary $\partial U$ which is homologous to the one-cycle $[z]$. Let $z_{\alpha}, z_{\beta}$ be one-periodic orbits of Hamilton's equation 
for $H_{1}, H_{2}$ respectively such that the images of $z_{\alpha}$ and $z_{\beta}$ are contained in an open subset

$$
V \subset \bar{V} \subset U
$$

and denote by $u_{\alpha \beta}^{c a n}$ be the canonical thin cylinder. Suppose that $u$ is a solution of (5.3) satisfying $u \sim u_{\alpha \beta}^{c a n}$

$$
u(-\infty)=z_{\alpha}, \quad u(\infty)=z_{\beta} .
$$

Then there exists a positive constant $\delta_{0}>0$ and $e=e\left(z ; \delta_{0}, V \subset U\right)>0$, which does not depend on $u$, such that for any finite energy solution $u$ of (5.3)-(5.4) whose image is not contained in $U$, we have

$$
E_{(j, \mathcal{H}, \rho)}(u) \geq e\left(z ; \delta_{0}, V \subset U\right)>0
$$

for any $H$ and $\mathcal{H}$ as long as $H_{m}, m=1,2$ satisfy

$$
\left\|H_{m}-H\right\|_{C^{2}}<\delta_{0}, \quad\|\mathcal{H}\|_{C^{2}}<\delta_{0}, \quad\left\|j^{\prime}-j\right\|_{C^{1}}<\delta_{0}
$$

Proof. We will prove the lemma by contradiction. Suppose to the contrary that there exists a sequence $\delta_{k} \rightarrow 0$ such that we can find $H_{\alpha, k}, H_{\beta, k}$ and $\left(j_{k}, \mathcal{H}_{k}\right)$ satisfying

$$
\left\|H-H_{\alpha, k}\right\|_{C^{2}}, \quad\left\|H-H_{\beta, k}\right\|_{C^{2}}, \quad\left\|\mathcal{H}_{k}-\mathcal{H}\right\|, \quad\left\|j_{k}-j\right\|<\delta_{k}
$$

for which there exist periodic orbits $z_{\alpha, k} \in \operatorname{Per}\left(H_{\alpha, k}\right)$ and $z_{\beta, k} \in \operatorname{Per}\left(H_{\beta, k}\right)$ satisfying

$$
\operatorname{Im} z_{\alpha, k}, \quad \operatorname{Im} z_{\beta, k} \subset V,
$$

and a solution $u_{k}$ of (5.3)-(5.4) satisfying

$$
u_{k}(-\infty)=z_{\alpha, k}, \quad u(\infty)=z_{\beta, k}
$$

and

$$
u\left(\tau_{k}, t_{k}\right) \notin U .
$$

We recall the energy bound $(5.21)$

$$
E_{\left(j_{k}, \mathcal{H}_{k} ; \rho\right)}\left(u_{k}\right) \leq \mathcal{A}_{H_{\beta, k}}\left(\left[z_{\beta, k}, w_{k} \# u\right]\right)-\mathcal{A}_{H_{\alpha, k}}\left(\left[z_{\alpha, k}, w_{k}\right]\right)+E^{-}\left(H_{\beta, k}-H_{\alpha, k}\right)
$$

for any bounding disc $w_{k}$ of $z_{\alpha, k}$. Since $u$ is assumed to be homotopic to the canonical thin cylinder $u_{\alpha \beta}^{c a n}$, we have

$$
\mathcal{A}_{H_{\beta, k}}\left(\left[z_{\beta, k}, w_{k} \# u\right]\right)=\mathcal{A}_{H_{\beta, k}}\left(\left[z_{\beta, k}, w_{k} \# u_{\alpha \beta}^{c a n}\right]\right)
$$

and hence this bound can be re-written as

$E_{\left(j_{k}, \mathcal{H}_{k} ; \rho\right)}\left(u_{k}\right) \leq \mathcal{A}_{H_{\beta, k}}\left(\left[z_{\beta, k}, w_{k} \# u_{\alpha \beta}^{c a n}\right]\right)-\mathcal{A}_{H_{\alpha, k}}\left(\left[z_{\alpha, k}, w_{k}\right]\right)+E^{-}\left(H_{\beta, k}-H_{\alpha, k}\right)$.

Using (A.8)-(A.10) and Lemma 2.4, we derive

$$
E_{\left(j_{k}, \mathcal{H}_{k} ; \rho\right)}\left(u_{k}\right) \rightarrow 0 \quad \text { as } \quad k \rightarrow \infty .
$$

In particular, $u_{k}$ can not bubble off and uniformly converges to a stationary solution. We denote the stationary solution by $z_{\infty}$, which will be a periodic 
orbit of $H$. Due to (A.9) and (A.10), the image of $z_{\infty}$ must be contained in $\bar{V}$. On the other hand, because of (A.11), the image of the limit of $u_{k}$ cannot be contained in $U$ and gives rise to a contradiction. This finishes the proof.

Now we prove Lemma 6.8 by contradiction. Suppose the contrary that there exists some sequence of Floer points $\eta_{i}$ with $\operatorname{dist}\left(\eta_{i}, \mathcal{S i n g}(\mathcal{H})\right) \rightarrow 0$ and a sequence of elements

$$
u_{i} \in \mathcal{M}\left(J\left(\eta_{i}\right), H\left(\eta_{i}\right) ;\left[z^{+}\left(\eta_{i}\right), w^{+}\left(\eta_{i}\right)\right],\left[z_{i}, w_{i}\right]\right)
$$

with $\left[z_{i}, w_{i}\right] \neq\left[z^{-}\left(\eta_{i}\right), w^{+}\left(\eta_{i}\right) \# u_{z^{+} z^{-}}^{c a n}\right]$ and

$$
E_{\left(J\left(\eta_{i}\right), H\left(\eta_{i}\right)\right)}\left(u_{i}\right) \rightarrow 0 .
$$

After choosing a subsequence, we may assume

$$
\eta_{i} \rightarrow \eta_{\infty} \in \operatorname{Sing}(\mathcal{H})
$$

and

$$
z_{i} \rightarrow z_{\infty} \in \operatorname{Per}\left(H\left(\eta_{\infty}\right)\right)
$$

as $i \rightarrow \infty$.

First if $z_{i} \neq z^{ \pm}\left(\eta_{i}\right)$, then we have

$$
d_{C^{1}}\left(z_{i}, z^{ \pm}\left(\eta_{i}\right)\right) \geq C
$$

for some $C>0$ independent of $i \rightarrow \infty$ because we are assuming $\mathcal{H}$ is a Cerf homotopy and so each singular point $\eta_{\infty}$ can contain only one bifurcation orbit. This contradicts to (A.11) via Lemma A.3.

On the other hand, if $z_{i}=z^{+}\left(\eta_{i}\right)$, (A.11) implies $\operatorname{Im} u_{i}$ is contained in a small tubular neighborhood of $z_{\infty}$ and so the compactified cycle $u_{i}$ are all homologous to the one dimensional cycle $z_{i}$. But then Lemma 6.3 implies

$$
E_{\left(J\left(\eta_{i}\right), H\left(\eta_{i}\right)\right)}\left(u_{i}\right)=0
$$

for all sufficiently large $i$, after choosing a subsequence if necessary, which contradicts that $u_{i}$ cannot be stationary.

Therefore we have proved

$$
z_{i}=z^{-}\left(\eta_{i}\right)
$$

for all $i$, after choosing a subsequence if necessary. Then we pick a pair of sufficiently small tubular neighborhood $U$ of $\operatorname{Im} z_{\infty}$ such that it deformation retracts to the one dimensional cycle $z_{\infty}$. Since $z_{i} \rightarrow z_{\infty}$, there exists another smaller open neighborhood $V$ with $\bar{V} \subset U$, which depends only on $z_{\infty}$ and contains the image of $z_{i}$ for all sufficiently large $i$. We can also make the energy in (A.11) satisfy

$$
E_{\left(J\left(\eta_{i}\right), H\left(\eta_{i}\right)\right)}\left(u_{i}\right)<e\left(z_{\infty} ; \delta_{0}, V \subset U\right)
$$

by choosing $i$ sufficiently large. Then Lemma A.3 implies that the image of $u_{i}$ must be contained in $U$. In particular, $z^{+}\left(\eta_{i}\right)=u_{i}(-\infty)$ itself must be 
contained in $U$. In particular, after compactifying $\mathbb{R} \cup\{-\infty, \infty\}$ to $[0,1]$, we prove that $u_{i}$ must be homotopic to $u_{z^{+} z^{-}}^{c a n}$. This proves

$$
\left[z_{i}, w_{i}\right]=\left[z^{-}\left(\eta_{i}\right), w^{+}\left(\eta_{i}\right) \# u_{z^{+} z^{-}}^{c a n}\right]
$$

which is exactly what we wanted to prove. This proves Lemma 6.8 .

\section{References}

[1] V. Benci and P. Rabinowitz, Critical point theorems for indefinite functionals, Invent. Math. 52 (1979), no. 3, 241-273.

[2] C. C. Conley and E. Zehnder, Morse-type index theory for flows and periodic solutions for Hamiltonian equations, Comm. Pure Appl. Math. 37 (1984), no. 2, 207-253.

[3] Y. Eliashberg and L. Polterovich, Partially ordered groups and geometry of contact transformations, Geom. Funct. Anal. 10 (2000), no. 6, 1448-1476.

[4] M. Entov, Commutator length of symplectomorphisms, Comment. Math. Helv. 79 (2004), no. 1, 58-104.

[5] A. Floer, The unregularized gradient flow of the symplectic action, Comm. Pure Appl. Math. 41 (1988), no. 6, 775-813.

[6] Morse theory for Lagrangian intersections, J. Differential Geom. 28 (1988), no. $3,513-547$.

[7] - Witten's complex and infinite dimensional Morse theory, J. Differential Geom. 30 (1989), 207-221.

[8] (1989), no. 4, 575-611.

[9] K. Fukaya, Y.-G. Oh, H. Ohta, and K. Ono, Lagrangian Intersection Floer Theory, book to appear, 2006, preprint.

[10] H. Hofer and D. Salamon, Floer homology and Novikov rings, The Floer memorial volume, 483-524, Progr. Math., 133, Birkhauser, Basel, 1995.

[11] Y. J. Lee, Reidemeister torsion in Floer-Novikov theory and counting pseudo-holomorphic tori, I, J. Symplectic. Geom. 3 (2005), no. 2, 221-311.

[12] D. McDuff, Geometric variants of the Hofer norm, J. Symplectic Geom. 1 (2002), no. $2,197-252$.

[13] J. Milnor, Lectures on the h-Cobordism Theorem, Notes by L. Siebenmann and J. Sondow Princeton University Press, Princeton, N.J. 1965.

[14] Y.-G. Oh, Floer cohomology, spectral sequences, and the Maslov class of Lagrangian embeddings, Int. Math. Res. Not. IMRN (1996), no. 7, 305-346.

[15] _ Chain level Floer theory and Hofer's geometry of the Hamiltonian diffeomorphism group, Asian J. Math. 6 (2002), no. 4, 579-624.

[16] Normalization of the Hamiltonian and the action spectrum, J. Korean Math. Soc. 42 (2005), no. 1, 65-83.

[17] - Spectral invariants and the length minimizing property of Hamiltonian paths, Asian J. Math. 9 (2005), no. 1, 1-18.

[18] Construction of spectral invariants of Hamiltonian paths on closed symplectic manifolds, The breadth of symplectic and Poisson geometry, 525-570, Progr. Math., 232, Birkhauser Boston, Boston, MA, 2005.

[19] Spectral invariants, analysis of the Floer moduli space, and geometry of the Hamiltonian diffeomorphism group, Duke Math. J. 130 (2005), no. 2, 199-295.

[20] L. Polterovich, The geometry of the group of symplectic diffeomorphisms, Lectures in Mathematics ETH Zurich. Birkhauser Verlag, Basel, 2001.

[21] D. Salamon and E. Zehnder, Morse theory for periodic solutions of Hamiltonian systems and the Maslov index, Comm. Pure Appl. Math. 45 (1992), no. 10, 1303-1360.

[22] M. Usher, Spectral numbers in Floer theories, arXiv:0709.1127, preprint, 2007. 
[23] C. Viterbo, Symplectic topology as the geometry of generating functions, Math. Ann. 292 (1992), no. 4, 685-710

[24] A. Weinstein, Bifurcations and Hamilton's principle, Math. Z. 159 (1978), no. 3, 235248.

Department of Mathematics

UNIVERSITY OF WISCONSIN

MADISON, WI 53706, USA

AND

Korea Institute for Advanced Study

Seoul 130-722, Korea

E-mail address: oh@math.wisc.edu 